JAMES R. IRVINE

\title{
FORE TO FORM
}

Thesis supervisor: Bettina Neu

MASTER OF DESIGN INNOVATION

VICTORIA UNIVERSITY OF WELLINGTON

SCHOOL OF DESIGN

2015 


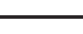

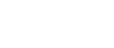




\title{
FORE TO FORM
}

\author{
JAMES ROBERTSON IRVINE
}

A 120-point thesis submitted to the Victoria University of Wellington in fulfilment of the requirements for the degree of Master of Design Innovation

Victoria University of Wellington

School of Design

2015

- III - 


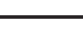

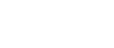


-

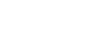




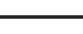

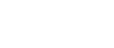




\section{PREFACE}

There is an old Chinese saying, "Sitting man must hold mouth open very long time before roast goose flies in." As foolish as this sounds, the words carry a lot of purpose. The underlying principle is that if you really want something, you must go out and get it. You can't achieve the impossible unless you try. This thesis is driven by these principles. It's subject is new and it's grounds are untested. It seeks to explore new possibilities that only design can offer. When we approach the unknown, success is never guaranteed.

But if we never try, we'll never know. 


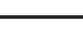

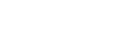




\section{ACKNOWLEDGEMENT}

I would like to acknowledge a number of people for their guidance and input throughout this research. Firstly my family, Bruce, Mary, Fran and Will for your encouragement, belief and support.

Secondly, Annabelle Nichols for your uplifting attitude in times of stress and your alternative yet professional design opinion. Paul, Deb and Gracie, thank you for your ongoing support and motivation to always go a little bit further.

A particularly important acknowledgment to my supervisor Bettina Neu for pushing me out of my comfort zone, testing my design skills and knowledge on every level and ultimately helping me become a better designer.

Margaret Petty, Nan O'Sullivan, Tim Miller, Jeongbin Ok and Simon Fraser for your individual views, wisdom and advice over the years. Dave, John, Graham, Gary and Phil from the workshop for your support and advice over the years. The office ladies for sorting all those annoying details, it makes life a lot easier.

Dev, Sarah and Sonja for your help with writing, it was invaluable. My friends, flatmates and classmates for their friendship and advice. In particular, Andrew Matautia for your help and skills behind a camera, you have the ability to capture everything I couldn't with words.

Tim Wigmore and Rebecca Asquith for your friendship and professional design advice, the skills you have taught me and the knowledge I've gained is something I'll treasure for the rest of my career.

Shout out to the guys at Customs and Scopa. Without the daily coffee fix and 'marghi' Tuesday's I doubt I would have made it through.

And finally Marco Duthie for being a daily inspiration. 


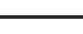

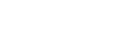




\section{CONTENTS}

PREFACE VII

ABSTRACT XIII

INTRODUCTION 3

$\begin{array}{ll}\text { SITE ANALYSIS } & 17\end{array}$

PROGRAMME ANALYSIS 33

LITERATURE \& PROJECT REVIEW 49

PRELIMINARY EXPERIMENTS 75

APPLIED EXPERIMENTS 135

$\begin{array}{ll}\text { PRELIMINARY DESIGN } & 151\end{array}$

DEVELOPED DESIGN 179

$\begin{array}{ll}\text { DISCUSSION } & 201\end{array}$

$\begin{array}{ll}\text { FIGURE LIST } & 205\end{array}$

REFERENCE LIST 209 


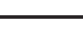

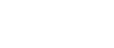




\section{ABSTRACT}

Designing for sports equipment demands excellence. The sheer nature of competition drives athletes to achieve the unachievable. This obsession to improve shifts from the athlete to the designer. The continual development and availability of materials, technologies and processes makes the role of the designer more critical than ever. Though the one real opportunity for innovation lies in how the designer interprets and utilizes these technologies.

The question that this research asks is: Can the integration and synchronisation of contemporary digital tools reshape the design process of golf clubs? This investigation predominantly uses an experimental 'research through design' approach based on the ideas and methods derived from a number of professional design projects and theoretical design approaches.

It argues that the unique combination and application of emerging digital tools can expose a breadth of creative design opportunities for golf club design. Golf clubs, like any other sports equipment must be designed with the underlying, crucial theme of performance improvement. The term performance can be broken down into two aspects; mental (visual) and physical (functional). The criteria for these aspects changes with each individual and demands a new level of customisation.

This thesis investigates how this could be achieved and proposes innovative pathways to integrate individual performance data as form defining inputs. It also explores the potential of new digital aesthetics to enhance functional criteria yet preserving critical features of traditional club design. 


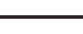

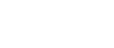


'It is not craft as 'handcraft' that defines contemporary craftsmanship: it is craft as knowledge that empowers a maker to take charge of technology” - Dormer, 1997. 


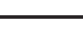

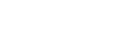


CHAPTER ONE

INTRODUCTION 


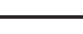

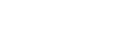




\section{AREA OF INTEREST}

As designers, we are continually presented with new tools in the form of technologies driven by science and engineering that allow us to explore new paths and potential design opportunities. Digital technologies offer designers an amazing level of fluidity in terms of inventiveness and innovation.

It is now easier than ever to explore, exploit and adopt these technologies and apply them to enhance our practise and introduce new ways of working (Shillito, 2013).

This divergent approach to designing sports equipment presents new possibilities in both form and aesthetic as well as function. Visually, the new generation of digital modelling programs allow us to generate intricate forms with relative ease. These complex geometries present designers with additional ways of perceiving objects, giving the designer more freedom to experiment with novel approaches to fulfilling functional requirements.

One advantage of digital tools is their ability to replicate real life simulations through mathematical function. This means we can quickly and effectively test prototypes, without the need to constantly fabricate and trial real world scenarios, which can save both time and money. This revolutionary aspect of digital technologies provides new perspective on the relationship between humans and objects.

This investigation seeks to expose new opportunities in the design and fabrication of sports equipment, driven by revolutionary capabilities of emerging technologies as design tools. It will consider the importance of both aesthetic and functional 
Fig. $1 \mid$ The Lotus Type 108 bicycle

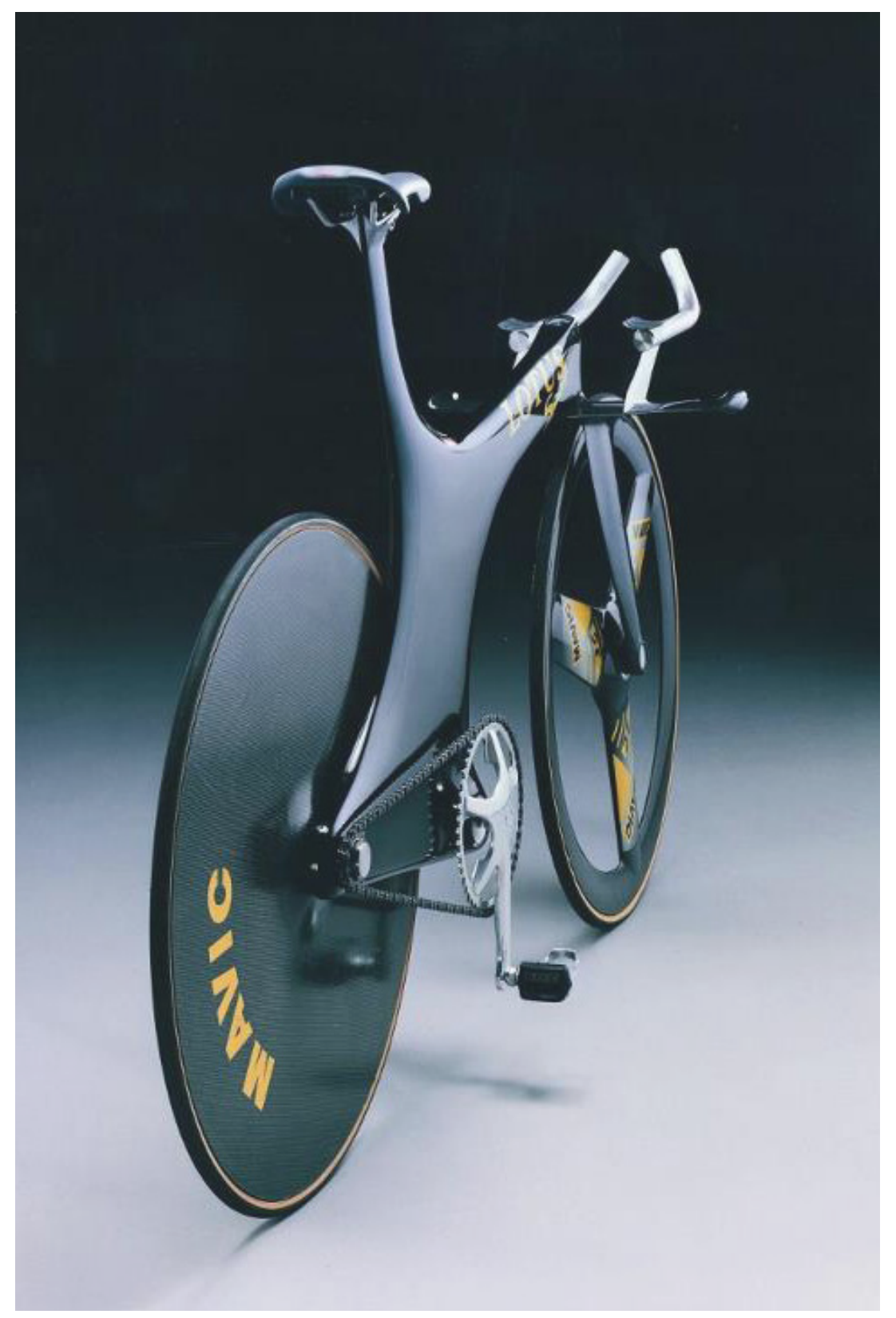

$-4-$ 
elements of sports equipment and aims to reshape our current perception of these elements as design features. In a similar manner to any other product development process, the design of sporting equipment involves an endless cycle of innovative concepts and user testing/feedback of both functional and aesthetic performance over numerous iterations and prototypes. However, dissimilar to many other product development processes, sports equipment must be designed with the underlying, critical theme of performance improvement. Performance of sports equipment can be broken down into two categories; mental and physical.

Physical performance of sports equipment, is directly related to the functional aspects of a design and its success can be measured through the improvement of an athlete's 'on-field' performance, when said equipment is used.

Mental performance often relates directly to the aesthetics, design and form of an object. Particular details are purposefully emphasised or accentuated to enhance certain notations and feelings. As discussed by Noble \& Bestley (Noble \& Bestley, 2005) "Some objects and visual elements might relate directly to the envisaged resolution, whilst others are incorporated to denote emotional aspirations and the underlying feelings which the product is intended to evoke." Emphasising that these features can be both explicit and subliminal in nature.

An example that effectively demonstrates both physical and mental performance, is the Lotus Type 108 bicycle (fig 1). The Type 108 is an Olympic-class, individual pursuit bicycle whose radically innovative design redefined the 


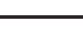

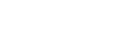


performance level of track cycling. The improvement in physical performance of this bicycle was unmistakable. Used at the 1992 Barcelona Olympics, English track cyclist Chris Boardman shattered the $4000 \mathrm{~m}$-pursuit event by a remarkable 4 seconds (Drimmer, 2014). The effect on mental performance from this design was also incredible. The bizarre, monocoque styled frame enticed significant interest from the media and other cycling teams. It's unique, functional style and bare, carbon-fibre aesthetic promoted a sleek efficiency and a ruthlessly fast aura. A revolutionary design that transformed sporting enterprise of cycling.

This investigation is particularly interested in the design, development and personalisation of golf clubs, particularly golf clubheads. More specifically this research focuses directly on the putter. While no club, according to the rules of golf (USGA, 2015) is completely indispensable from a player's bag, the putter is the closest. It's highly specialised nature means virtually no player is without one.

The golf clubmaking process has seen the introduction of a vast range of technologies and materials over time which in turn have presented new design possibilities. This research looks to take this process one step further through the implementation of emerging digital technologies. It will explore current applications for such technologies, the advantages unique to their application and where (if possible) these advantages could improve the golf club design process. 


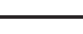

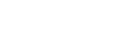


Can the integration and synchronisation of contemporary digital technologies reshape the design process of golf clubs? 


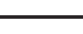

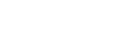




\section{AIMS \& OBJECTIVES}

The principal aim driving this thesis is to investigate how the novel application of contemporary digital tools, technologies and processes can influence the golf club design process, challenging current perceptions of functional criteria for the customisation of golf clubs.

The principle objectives of this thesis are to develop a series of experimental design outputs that:

1. Integrate and synchronise emerging digital tools in the ideation phase of the design process

2. Utilize individual performance data as form defining inputs

3. Explore the potential of new aesthetics to enhance functional criteria

4. Preserve critical features of traditional golf club design. 
"Research through design takes advantage of the unique insights gained through design practice to provide a better understanding of complex and future-oriented issues in the design field." - Godin \& Zahedi, 2014 


\section{DESIGN PROCESS}

This thesis is a design-led research investigation. "Design research is not concerned with what exists but with what ought to be" (Milton \& Rodgers, 2013). As discussed by Milton \& Rogers, design research has evolved into three distinct forms: research about design (history, theory and context), research as design (innovative design methods) and research through design (experimental practise). Each of these categories is equally important in terms of what they achieve in the design process. However, due to the iterative and exploratory nature of methods and tools used in the concept and development of this investigation, a research through design heavy approach has been adopted, or more specifically, research through digital design. 
Chapter one (introduction) discusses the relationship between design, sports and technology and the potential of emerging technology for design development of sports equipment. It introduces the aspects of performance that are addressed through design, and the specific subject of exploration. It details the the main research question and the fundamental aims and objectives of this exploration.

Chapter two (site analysis) analyses and articulates empirical issues in building a specific criteria to be addressed throughout the design experiments. These issues have been divided into five segments. The first three segments cover the history and development over time of sports, golf, golf equipment, and the putter. The analysis of the putter is notably important as it identifies the purpose, context and functional components that drive the design experiments. The last two segments describe the two most important aspects within putter design; weight distribution and customisation. Weight distribution covers vital, functional aspects of putter design, while mass customisation suggests how this could be tailored to the individual.

Chapter three (programme analysis) articulates programme-specific items (contemporary digital technologies are outlined in chapter one), that are to be exploited throughout the design experiments. This chapter is broken into four segments. The first three segments analyse and review the potential advantages of digital technologies used through the design exploration. These technologies are: digital data collection tools, parametric modelling and additive manufacturing. Current contexts and applications of these tools are covered in greater detail in chapter four (project review). The final segment discusses the continually emerging computational design aesthetic and its evolution as a result of digital technologies.

Chapter four (literature \& project review) discusses both exemplary projects and literature that have been identified as relevant theoretical and inspirational inputs to technology driven experiments. The theoretical approaches are analysed and interpreted based on how they address the fundamental (functional and aesthetic) principles of this research while the project review assesses design approaches on their ability to produce practical solutions based on how digital tools are used in alternative context. Insights have then been translated through a series of visual methodologies and conclusions. Items such as the Digital Form Guide were developed as a unique approach to deconstructing new aesthetics while also acting as a guide to drive digital experiments.

Chapter five (preliminary experiments) covers the early investigation of design experiments testing theoretical and functional aspects identified in chapter two through the mediums (digital tools) described in chapter three. 
These initial design experiments introduce and test the research through design approach. Each experiment series targets specific features exclusive to digital technologies driven by an abstract differentiated approach to developing design solutions recognised in chapter two. Once tested, each series is assessed on its strengths and weaknesses, and in conjunction with the vision of the designer, evolves into the next stage series of experiments. Individual experiments are presented as an iterative series to aid in understanding the context, purpose and sequential process.

Chapter six (applied experiments) describes initial design experiments that combine further practical, functional clubhead aspects (such as loft, lie, dimensions and geometry) as 3D modelling parameters with knowledge inherited from the preliminary experiments. This chapter explores how and where the integration of computational design aesthetics (from previous experiments) can be appropriately applied into a golf clubhead, to address both functional and aesthetic performance factors. This chapter also begins to explore the application of individual user data to drive these parameters, addressing customisation in both performance factors elements.

Chapter seven (preliminary designs) covers five preliminary design outputs that each take a divergent approach based on outputs and conclusions from the previous two chapters. Each design showcases a unique aesthetic explored throughout the preliminary experiments and inspired by the digital form guide. Each design also inherits a broad set of data, recorded using digital data tools, to suit five unique stroke (putting) styles. The result is five custom designed putters that briefly address unique aesthetic preferences as well as practical, data driven (functional) clubhead aspects.

Chapter eight (developed design) further explores one specific design output from chapter seven. The selected design was chosen based on its effectiveness to employ functional design aspects based on the designers individual swing (stroke) data, as well as aesthetic preference of the designer. This chapter further refines the application of swing data as parametric inputs to be more coherent with practical applications such as centre of gravity and moment of inertia.

Chapter nine (discussion) is a critical reflection on the process and outputs of this design investigation. It assesses the strengths and weaknesses discovered throughout the experimentation chapters, whilst offering a reflection on limitations and opportunities foreseen which could be further explored outside the scope of this thesis. It also discusses the feasibility of the end design output as functional object in the golfing/ sports industry. 


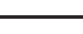

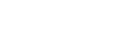


CHAPTER TWO

SITE ANALYSIS 
Fig. 2 | Illustration of Clisthenes, Olympic games

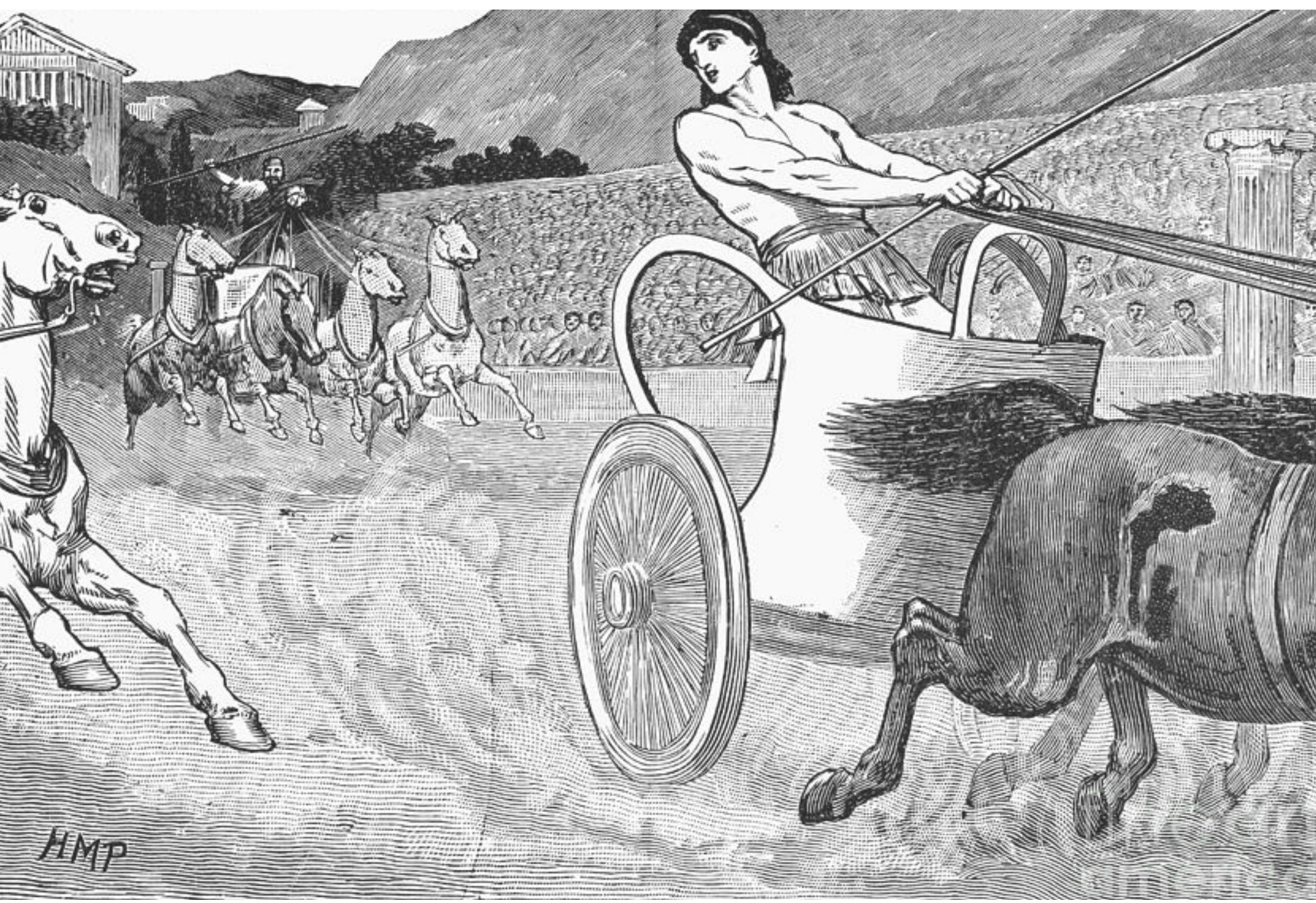




\section{EVOLUTION OF SPORT}

It's unclear when the idea of sport was first proposed or applied within a competitive setting. Presumably, it dates back to the existence of people as dynamic beings. Sports on a noncompetitive level, has been paramount to the evolution of primary human skills and development within nature and the environment. On a competitive level, it has pushed us to improve these skills further as we face new challenges and more fierce competition.

The professional era of modern sports has seen competition prosper and the development of sport become not just a hobby, but a viable career lifestyle choice. Intense competition has resulted in any form of advantage being seized to better an athlete's performance, including improved design of sports equipment.

One could say, the evolution of sports equipment is driven by our own desire to transcend the basic abilities offered by our physiques.

David Epstein points out, "sports technology has changed the face of performance, of all sports, from faster skis to lighter shoes." (Epstein, 2014). Epstein's example of Eddies Merckx's record for the longest distance cycled in one hour proves that the introduction of aerodynamics in bicycle design has not only changed the face of cycling, but provided an incomparable advantage to the modern day cyclist. 
Fig. 3 | Illustration of golf in the late, circa 1890

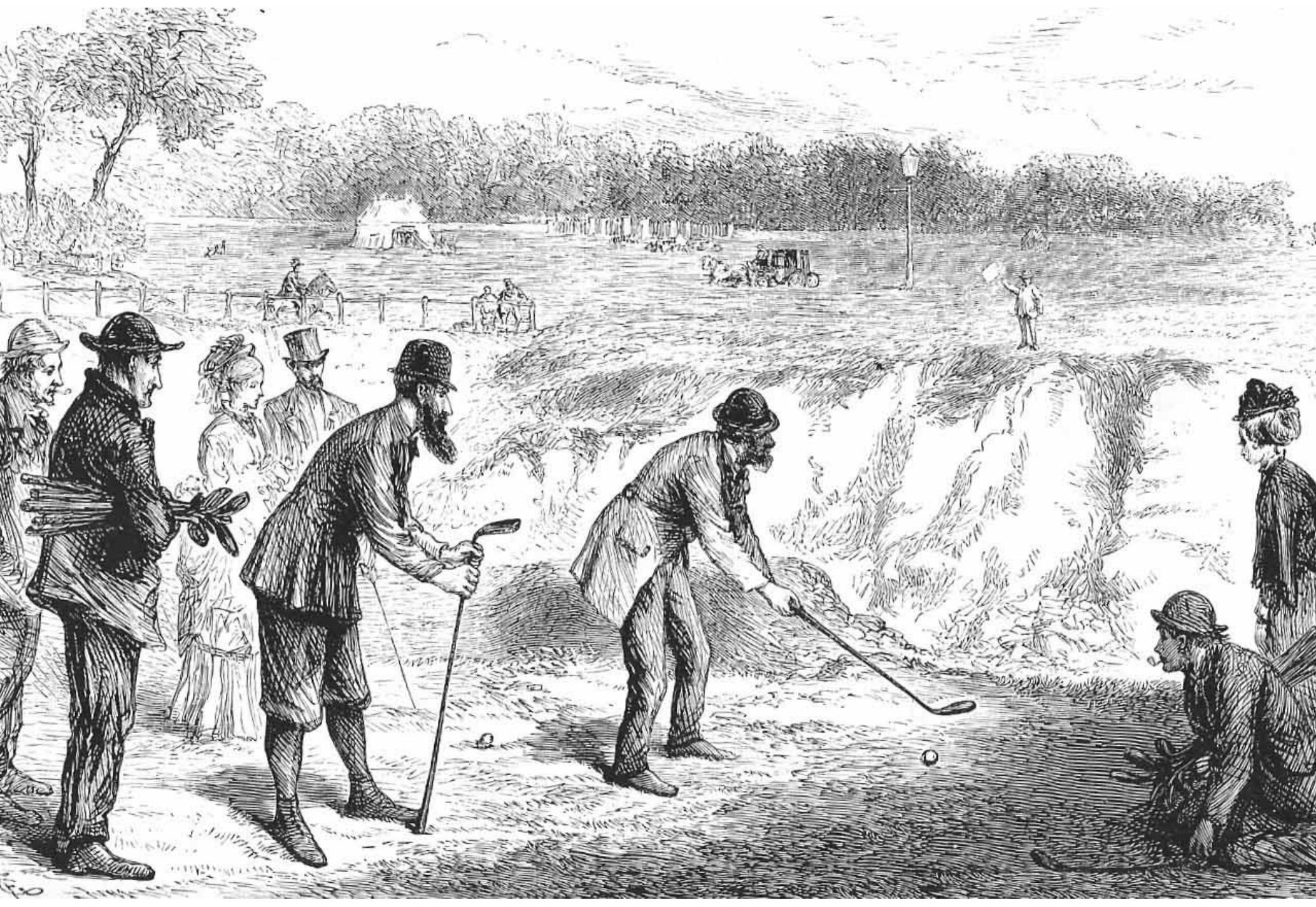




\section{EVOLUTION OF GOLF}

Golf can be traced back to the early 1200 's, where a similar game was played with a stick and a leather ball. However, the game as we know today, with a 'colf' (golf club), rather than a stick, is considered to have been birthed in Scotland during the 1400's (Bohn, 2007).

"Since golf's origin over 500 years ago, golf clubs have undergone tremendous change and development. Alongside improvements in materials, shapes and methods of manufacturing, a variety of ingenious clubs have been developed to encounter specific situations on the course." (Ellis, 1997). This evolutionary design process has seen the introduction of new technologies and materials, which in turn present new design possibilities.

Golf clubmaking can be described as an evolutionary design approach with a rich history of revolutionary innovative elements. The use of digital tools in the design and manufacturing process of golf clubs is therefore a logical evolutionary addition to existing fabrication techniques such as forging, casting and hand crafting. 
"A man's club's become almost members of his body, and certainly, if their capacity for conveying pleasure and pain to the senses is to measure the matter, the comparison is not overstrained." - Smith, 1908 
By exploring what contemporary technologies offer, we as designers, can respond to the needs and demands of modern golfers, which can be broken down in the following two categories:

Physical performance factors: how the club performs as a functional object, resulting in the change of an athlete's 'onfield' performance

Visual performance factors: how the club performs as an aesthetic object, resulting in the enhancement of particular notations or designed features.

One example is the introduction of titanium as a manufacturable material. Titanium's unique properties have resulted in innovative clubhead designs, and a new level of performance present in the modern game. As stated by Marc Jenkins (Jenkins, 2009), "titanium is 45 percent lighter than steel, therefore a titanium club does not surrender any power on a stroke because of the lightweight feel it provides." Titanium drivers are now standard in contemporary golf equipment. 
Fig. $4 \mid$ Illustration - Evolution of putter form
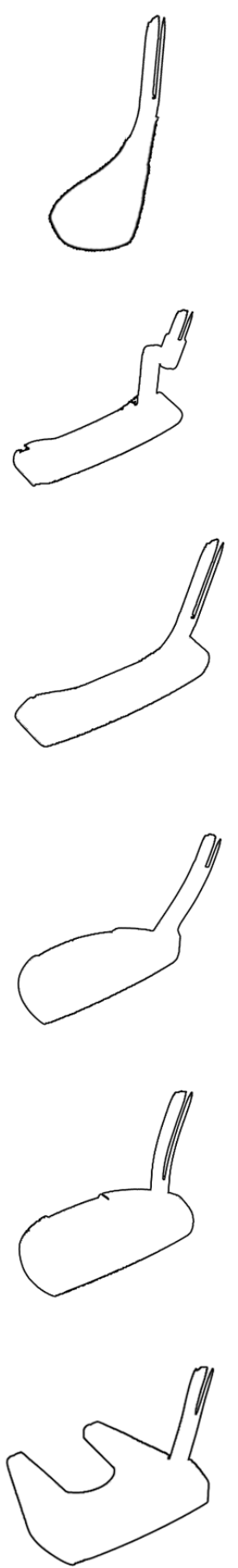


\section{THE PUTTER}

The putter is a golf club used specifically to make relatively short, low-speed strokes with the intention of rolling the ball into the hole, along the ground from a short distance. In comparison to other clubs (irons, wedges, and woods), the putter is unique in its form and function.

The clubhead often showcases a very flat, low profile and a low-loft striking face. However, this wasn't always the case (fig 4). The evolution of the putter in terms of materials and manufacturing processes is very similar to other club types. During the 16th century, the putter was fashioned out of hard-wood such as beech, and referred to as the 'putting cleek'. It wasn't until the first rubber ball, the 'guttie' was introduced in 1848 that putter heads were fabricated from metal (Bohn, 2007).

Golfers are forever experimenting with putters to find the one that feels absolutely perfect and gives them more confidence on the green - (Stetina, 2013).

The nature of putting makes it the most precise component of the game. Even the smallest of changes in accuracy, speed, and power can be the difference to whether a shot is made or not. Therefore a putter must be designed to provide the golfer with any technical advantage (stroke, glide, impact, spin) as well as promoting any technique improvement through customisation for individual stroke type. 

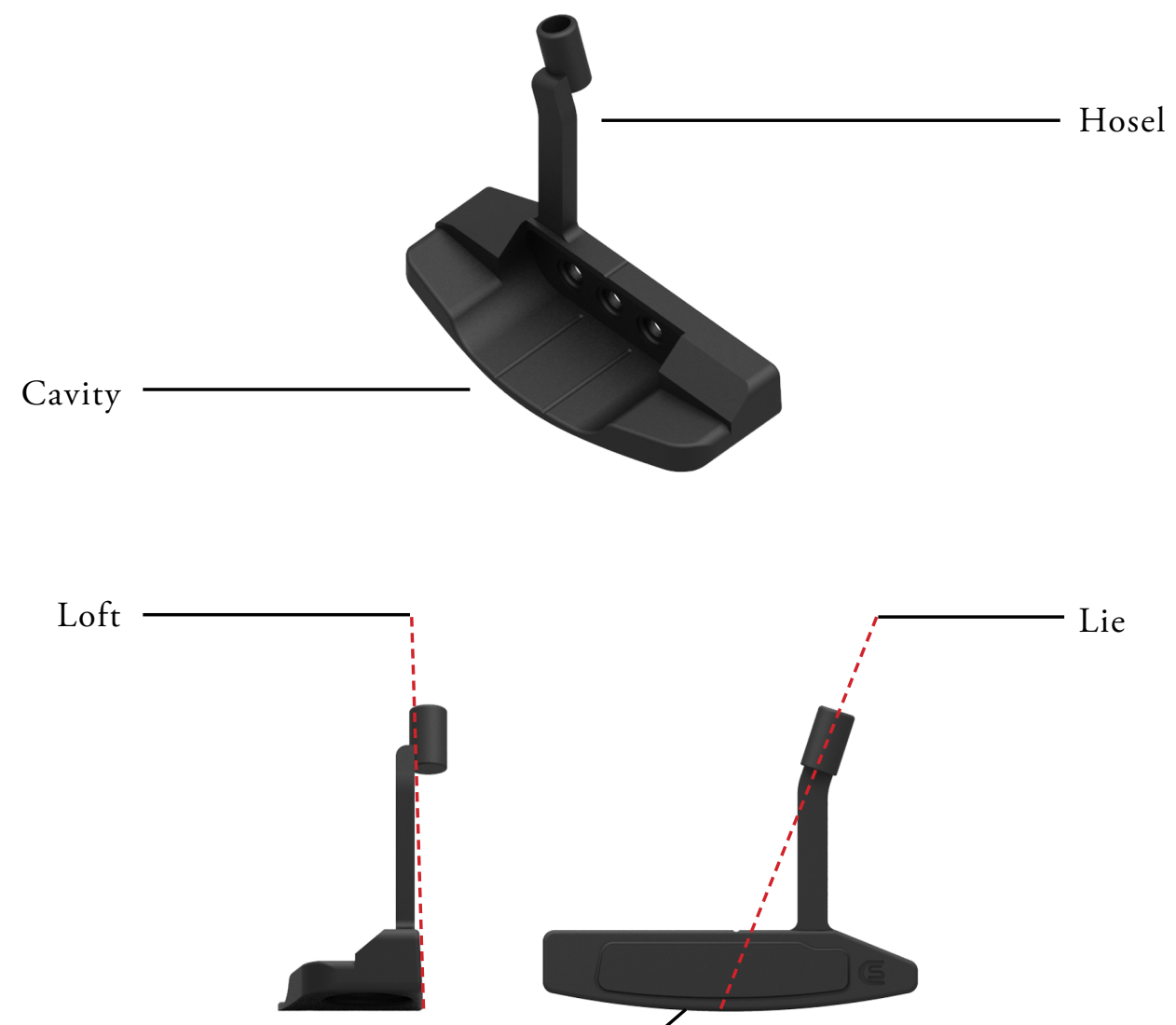

Sole
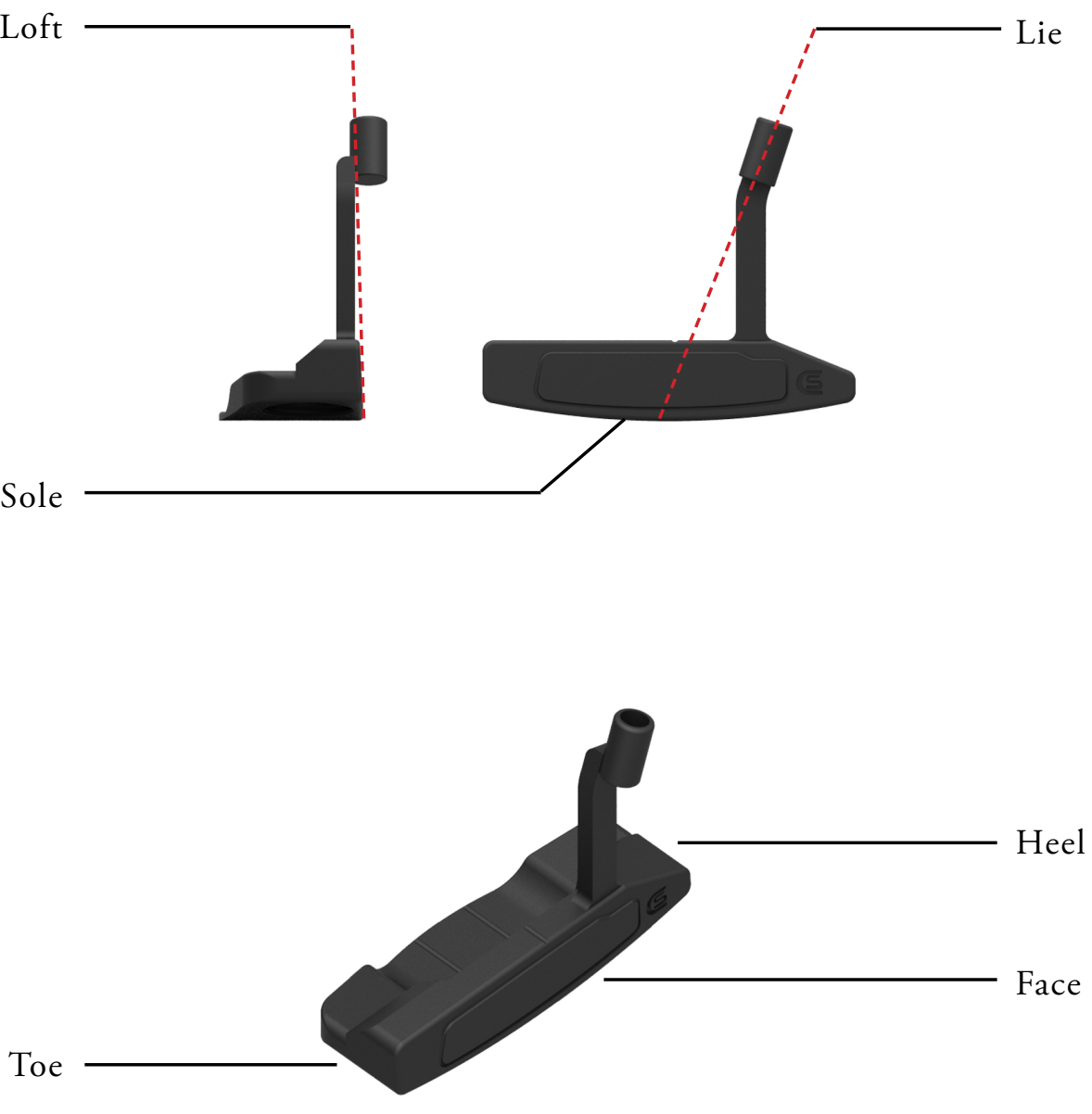
According to Ralph Maltby (Maltby, 2006) of Golfworks, commonly regarded as one of the premier golf equipment experts worldwide, there are five customisable variables that affect the distance and direction of your putt; shaft length, lie angle, loft angle, moment of inertia (MOI), and clubhead weight. Choosing the correct shaft length is based on the golfer's hand to eye placement in relation to the ball, so for the sake of this research, this study focuses on the four variables that directly affect the design of the clubhead. Customisation of these variables, or 'putter fitting' as it's known in the industry comes down to the individual golfer's stroke type.

Lie angle is entirely related to directional control. Variability in lie angle can determine whether a shot is pushed or pulled by changing the clubface plane.

Face loft angle is entirely related to distance control. Variability in loft angle determines the skid to roll percentage of your putt and can control whether you consistently hit the ball short or long.

Lie angle and loft angle are both limited to a single axis of rotation where the sum can either increase or decrease. On the other hand, due to the introduction of new technologies and rapidly changing levels of functional performance, moment of inertia and weight distribution have led to substantially more controlled clubhead weight and weight distribution. 
Fig. 6| High \& low MOI impact range

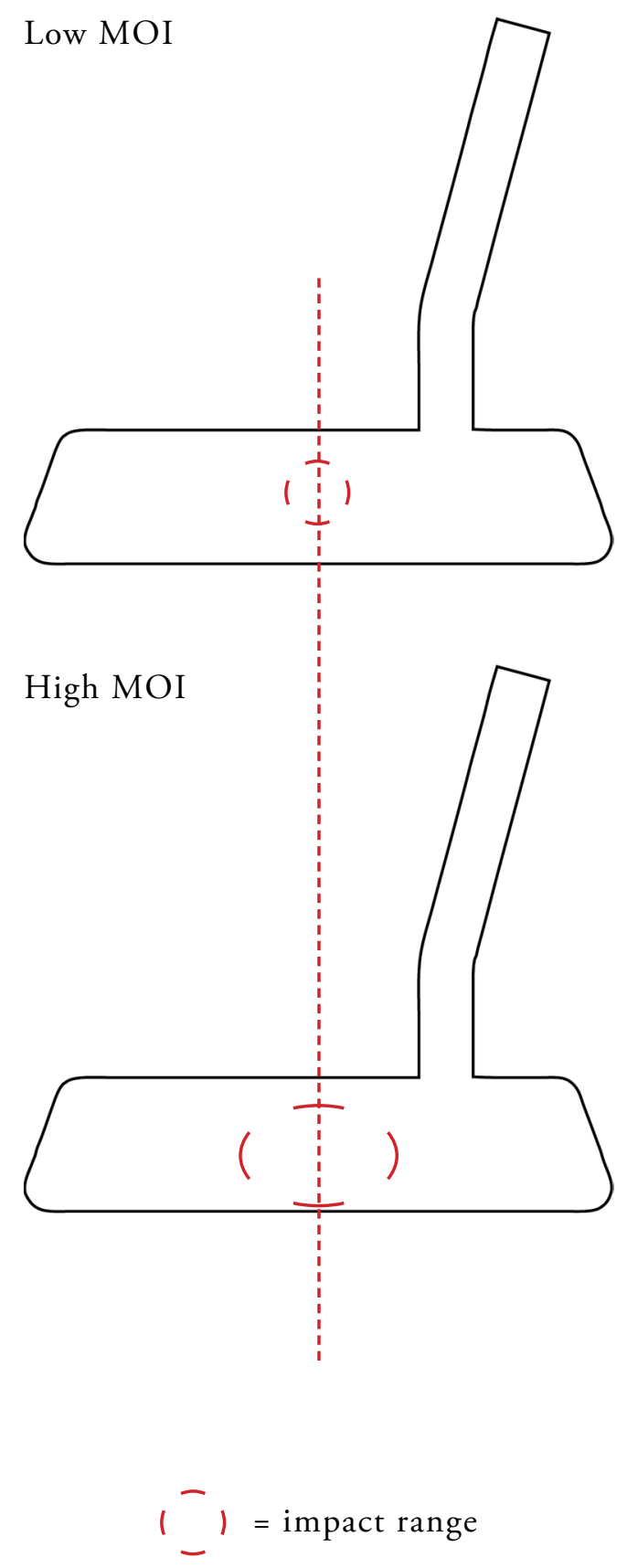




\section{WEIGHT DISTRIBUTION}

The distribution of weight is an essential aspect to consider when designing a clubhead for an individual. A precise, calculated distribution of weight suited to the player's stroke style can be vital in the success or failure of the design.

The most important variable in weight distribution is moment of inertia (MOI). MOI is a property of physics that indicates the relative difference in how easy or difficult it will be to set an object in motion about a defined axis of rotation. The higher the MOI of an object, the more force will have to be applied to set that object into rotational motion. Conversely, the lower the MOI, the less force needed to make an object rotate about an axis (Maltby, 2006).

When applied to a putting stroke, this essentially means the resistance to rotation created by off-centre hits. When the ball is struck from the centre of the club, the club doesn't rotate, and the ball goes straight. When the ball is struck on the toe, the toe kicks open and the ball is pushed away from the hole. When the ball is struck on the heel, the heel kicks open and the ball is pulled past the hole. This rule means that a consistent player, who always strikes the ball from the centre will have a low MOI (centered weighted club), whereas a less consistent player who strikes the ball all over the face will have a high MOI (perimeter weighted club). Impact range for wach type illustrated in fig 6 .

As discussed in The Ultimate Putting Program by Henry Stetina (Stetina, 2013), a professional golf instructor, a putter head requires ideal weight distribution for an individual's swing. He describes how "the combination of a golfer's stroke type and weight distribution of their putter determines their ability to control the speed and direction of their putts", proving the importance of customised or 'fitted' equipment. 
Fig. 7| Original Ping colour code chart

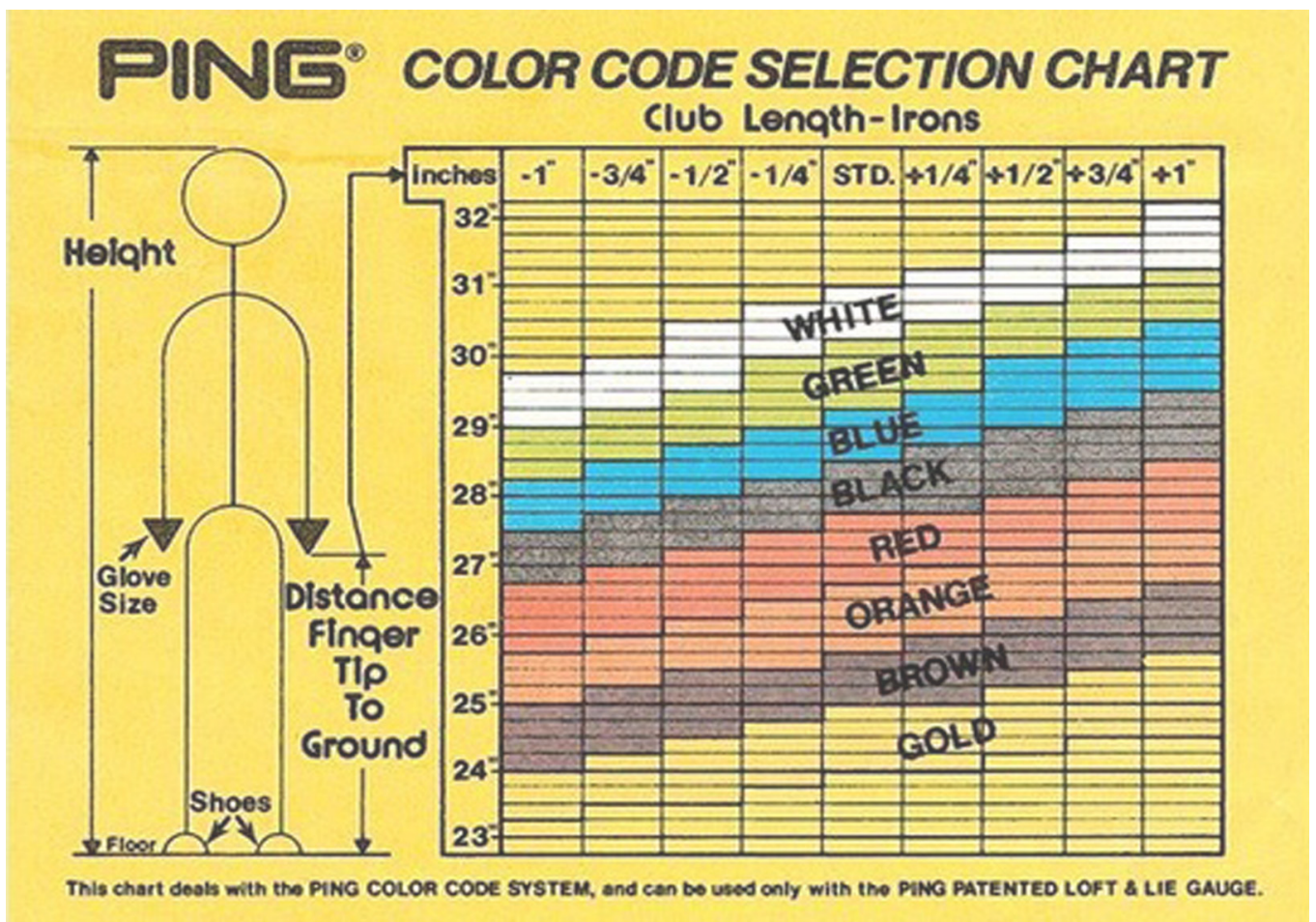




\section{MASS CUSTOMIZATION}

Customisation is the vanguard in business for both manufacturing and service industries. In production, mass customisation explores the middle ground between mass production and bespoke tailoring. The flexibility of computer-aided manufacturing systems has lead to the introduction of customised outputs for many products globally, combining the low cost of mass production with the adaptability of individualisation.

The nature of sport in the professional era has led to an increased level of competition and a burning desire to improve personal performance. Any advantage to improve performance is welcomed by an athlete, and this advantage can often come in the form of improved equipment and technology. In many sports, especially golf, ensuring that the equipment you use is designed to meet your individual needs is crucial to ensuring you can play your best, hence the increased demand for improved customisation of golf equipment.

The idea of mass customisation has trickled its way into the golf industry, with various companies exercising their own take on customisation. The earliest example of true customisation in the golf industry was the introduction of Ping's (one of the leading global golf equipment manufacturers) colour code system in 1972. Ping's new system encouraged every golfer from professional to amatuer to get 'fitted' clubs to play better and enjoy the game even more. Through the colour code system (fig 7), Karsten Solheim, founder of Ping, personalised the process of buying clubs for the first time. In today's industry, custom fitted clubs are the standard for all pro golfers as well as a large number of amateur golfers. 


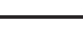

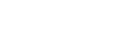


CHAPTER THREE 'PROGRAMME ANALYSIS' 
"Data is really the new voice of the athlete, and by listening to that voice, we can challenge the current paradigms and do something previously unimaginable" (Nurse, 2014). 
This chapter discusses the specific 'programme' of technologies that are used to explore the design criteria mentioned above, and to understand how they might allow the designer to modify, replicate, distribute and fabricate objects in ways we never thought possible. 
Fig. 8 | Swingbyte attached to club shaft

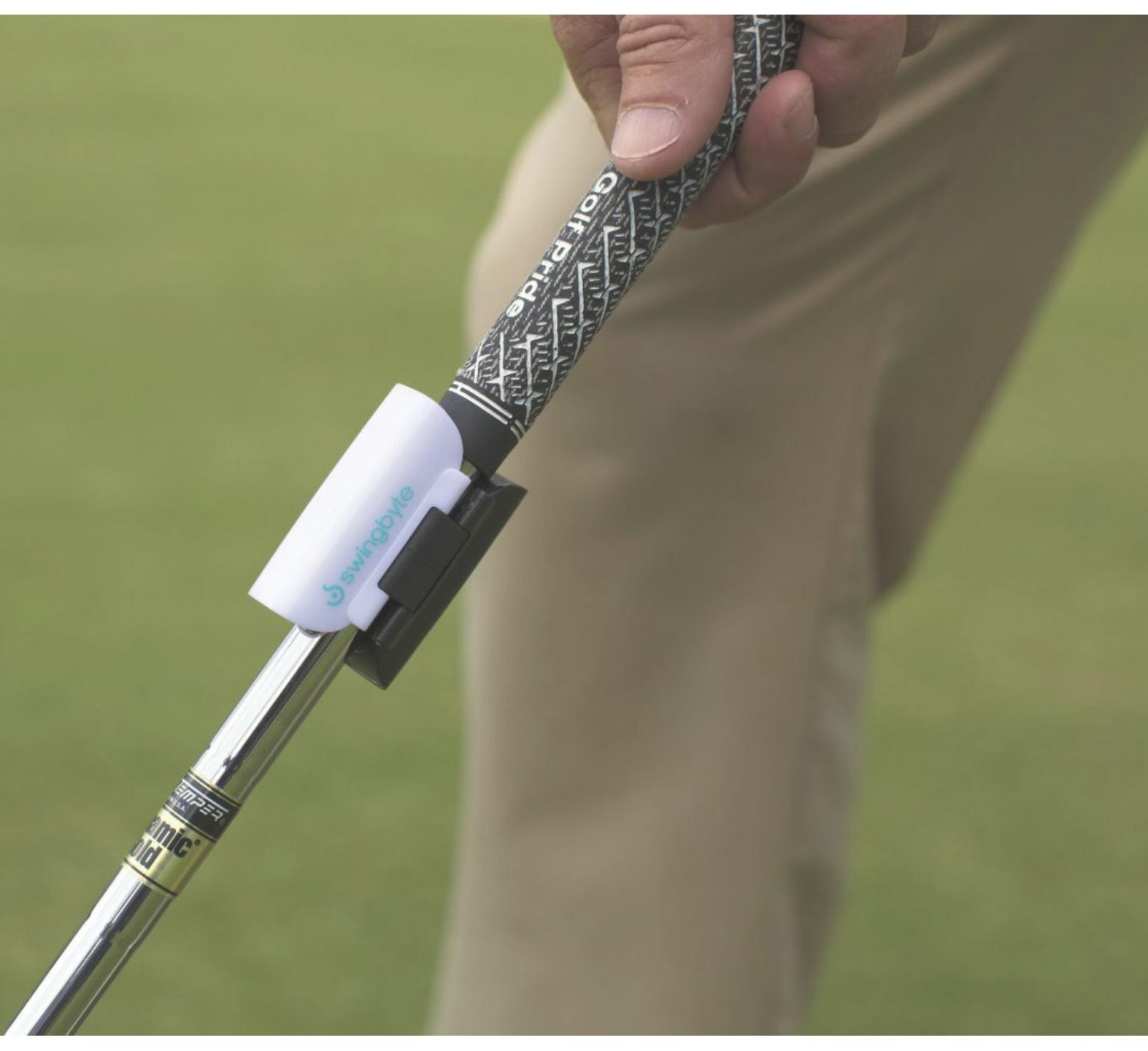




\section{DIGITAL DATA TOOLS}

The invention of mobile golf data tracking devices has resulted in a range of new tools that record, measure and display comparable data such as swing rhythm, tempo, speed as well as club plane angles, positions and direction. To appropriately determine effective data inputs, two data collection tools have been selected. Firstly, the Swingbyte 2 device (fig 8) which captures and displays individual golfers swing data in a visually easy to understand way. Simply attaching the device to the shaft on your club, below the grip allows the individual to play their natural swing without interference while simultaneously recording their swing/stroke data. The corresponding mobile app allows the user to select which club type and its specs to pick up data from driver to putter. The second tool is the iPing putting app, a wireless putting fitting and improvement tool. The app analyses and displays your putting consistency using three criteria: stroke type, impact angle, and tempo. The app monitors your consistency over time and gives you an average score.
We've done thousands of hours of player testing that show when a golfer's putter balance matches their stroke type, their consistency goes way up, and they make a lot more putts. (Solheim, 2015).

The iPing app isn't as accurate as the Swingbyte 2, however it is designed solely for measuring putting data whereas the Swingbyte is more precise, but also designed to accommodate every club type. Therefore data will be analysed as equally important from both and an average will be taken.

The Swingbyte 2, iPing and other similar devices are currently used by a vast number of professional golfers and coaches as a training tool to improve and monitor their swings using existing clubs. However, these tools have never been used to aid the design of the clubs themselves. The data from these devices will be analysed and translated into a medium that can aid the design of adjustable 3D modelling parameters. 
Fig. $9 \mid$ Typical parametric modelling interface

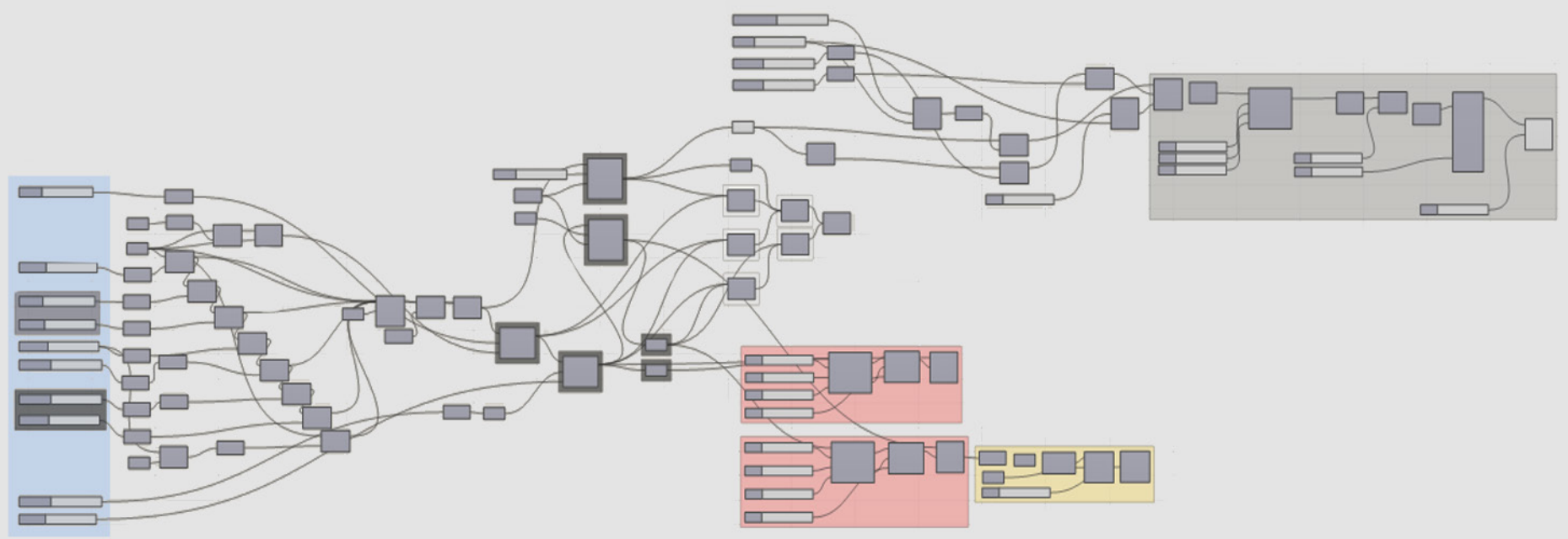

Individual data inputs

Distribution of weight

Form constraints

Club form output

Aesthetic output 


\section{PARAMETRIC MODELLING}

"A parametric solid can be defined as a solid whose actual shape is a function of a given set of parameters and constraints upon them. The objective of parametric modelling is to represent, manipulate, and reason in a computer about the three-dimensional (3D) shape of parameterised solid objects." (Hoffman \& Joan-Arinyo, 2002)

Parametric models are driven by relationships between components. These relationships are specifically chosen rules and constraints, which define aspects of a design. As discussed by Michael Kilkelly, "a parametric approach only works when the problem is well understood" (Kilkelly, 2015). Updating or modifying part of a model, has implications on the entire model as there is a complete bi-directional association between parts. Parametric modelling is often used to design objects based on real-world behaviours or attributes as models can adapt to conditions based on parameter and input choice.
Due to the association between parts, parametric modelling allows for numerous, rapid iterations of models (digital sketching) without the need to rebuild a new model each time. However, a parametric modeller must be aware of the characteristics of components and interactions between them in order to execute a model's potential effectively.

This research analyses and interprets the data taken from the devices mentioned above and merges this data against functional aspects of a putter. For example, if a player's data suggests that they consistently hit the toe of the face, weight would need to be distributed to form a toe weighted putter to counteract this. Fig 9 exhibits a typical parametric interface. This example is the model for preliminary design 5 . The colour code table shows the relationships between inputs, criteria and outputs. In relation to data driving function and aesthetic simultaneously. 
Fig. $10 \mid$ Intricate detail of Daniel Widrig's Grid

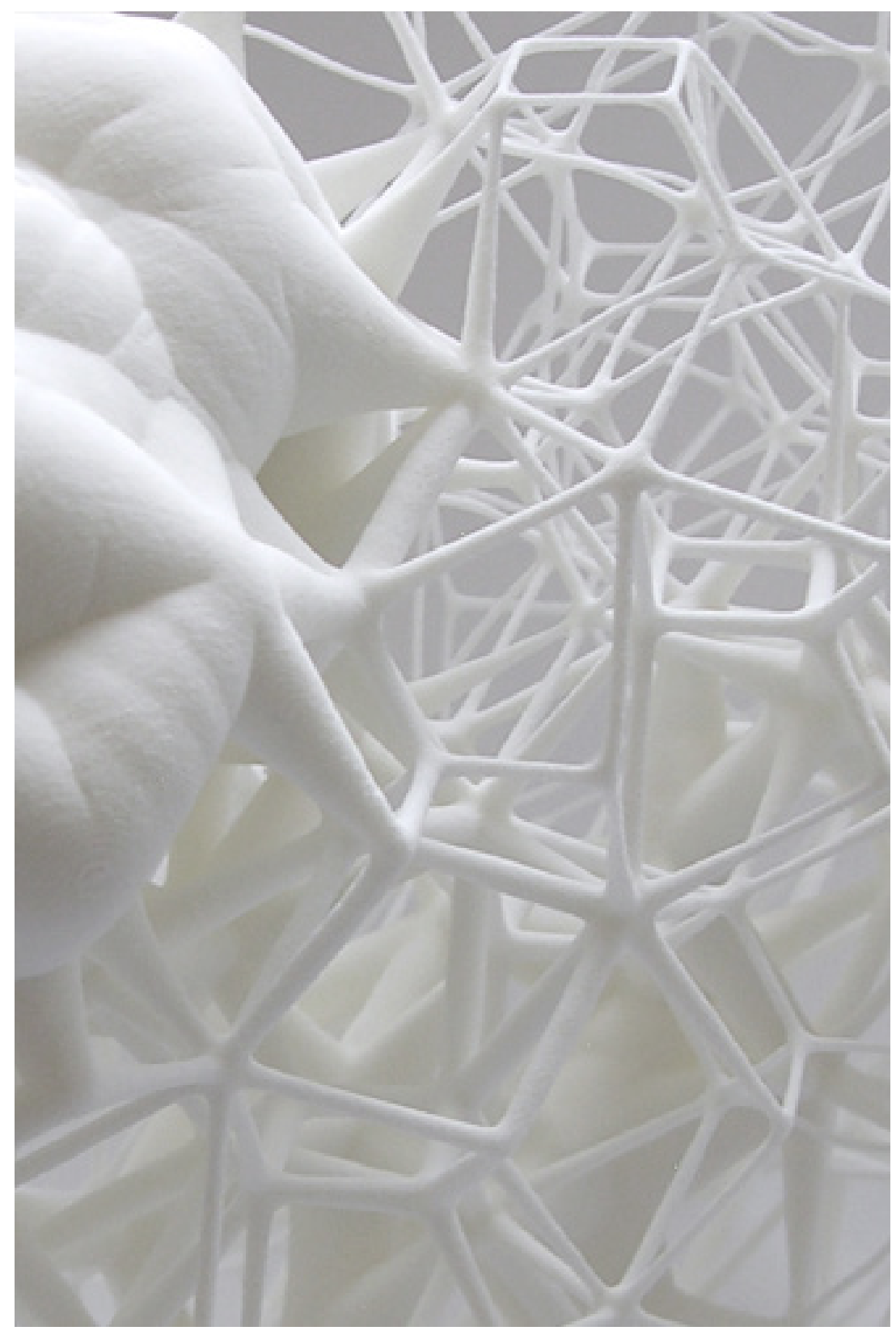




\section{ADDITIVE MANUFACTURING}

Additive Manufacturing (AM) provides the ability to fabricate almost any complex geometry (fig 10 ), using a range of materials and without great cost. The reduced need for existing tooling and efficiency of material make it a powerful tool in both manufacturing and design.

Joseph DeSimone, CEO of Carbon 3D (a revolutionary 3D printing company) describes the advantage additive manufacturing provides to produce intricate geometry through an experiment of his, "In its context it's not manufacturable by traditional manufacturing techniques. It has a symmetry which means you can't injection mould it, you can't even manufacture it through milling, therefore, this is a job for a 3D printer" (DeSimone, 2015).

Materials in AM are also developing at a rapid rate and now include the ability to print titanium and stainless steel, two of the fundamental materials currently used in the manufacturing of golf clubs. A study was undertaken in 2011 about the 'optimal design of a golf club using functionally graded porosity' (Ray, 2011). This engineering based study into the feasibility of electron beam melting (printing with powdered alloys), was an exploration into whether existing additive materials could be functionally used in golf clubs instead of traditional processes and materials. However, Ray's study was aimed at testing existing clubs designs. This thesis is distinguishable in the sense that it is driven to explore new possibilities in form and geometry.

Traditionally, manufacturing of golf clubs involved casting and/ or forging processes. These both limit the overall form that is to be fabricated as well as require large tooling costs. It is noted that there 
Fig. $11 \mid$ Zaanen, 3D printed putter
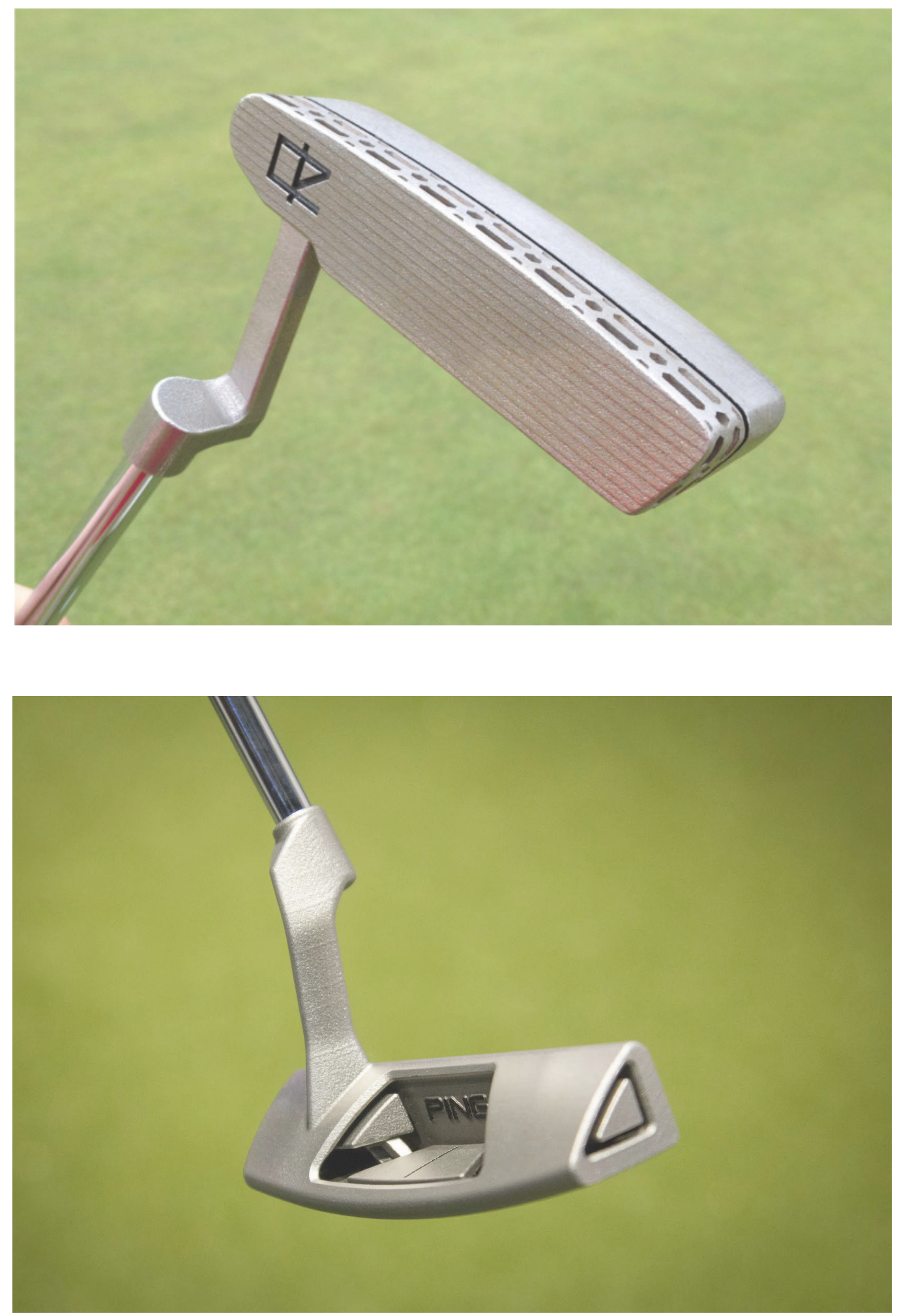

Fig. $12 \mid$ Ping, 3D printed putter 
is a gap where opportunity can be seen to push new functional aesthetics through input driven complex geometry. Due to the complex nature AM can offer there is opportunity to redefine traditional geometry and still comply with the USGA.

There are currently two known examples of metal, 3D printed putters. Both of these putters are excellent case studies for additive manufacturing, however theory application of other digital tools in less prominent. The first example (fig 11) by Zannen Putters explores "geometry that can only be created by 3D printing" (Zaanen, 2014) through a unique pattern behind the putting face. However, this example doesn't explore past the traditional form and exhibits no functional purpose behind the selected aesthetic.

The second example is Ping's 3D printed putter (fig 12). Ping have recently (Feb, 2015) announced their exploration into additive manufacturing through the release of this concept putter. This putter also takes a traditional approach in terms of shape with aspects of the putter having been designed to only be produced by additive manufacturing. However, in the opposite manner to Zannen Putter's design, Ping are much more focused on functional aspects therefore limiting their aesthetic exploration into what additive manufacturing could offer. (ref)

This research considers AM as a digital tool that allows for the production of complex forms to explore new digitally driven aesthetics. Salome Galjaard, an architectural engineer who's been working with AM and digital optimisation tools state's " $3 \mathrm{~d}$ printing will definitely have a big impact on the visual appearance of the world we live in." (Galjaard, 2014). 
Fig. 13 | Trabeculae by Shiro Studio (computational design) 


\section{COMPUTATIONAL DESIGN}

Computational design (aesthetic) is a direct result of the increased use of digital technologies as design tools (fig 13). It aims to bridge the gap between traditional product design thinking, natural design aesthetics and powerful mathematical digital tools.

Until now, products went through numerous conceptual and physical design processes that used valuable resources, of time and money. The continual development of applied emerging technologies has demonstrated the power of digital tools in design. Real world simulations and modifications are only a click away.

We as designers, can challenge old principles and break restrictions on form, geometry and aesthetic that were previously considered impossible or art. In the past, theories such as Louis Sullivan's Form follows Function (Sullivan, 1896) are challenged in the 21 st century. Rethinking the links between form and function and limitations of the past, is Sullivan's theory the only way to create meaningful design? This research poses the question, why can't form drive function?

The combination of digital 3D modelling software and computer-numerically controlled (CNC) manufacturing processes has seen the emergence of the trend that is, computational aesthetics. 
Fig. $14 \mid$ Hyphae 3D 1 by Nervous System

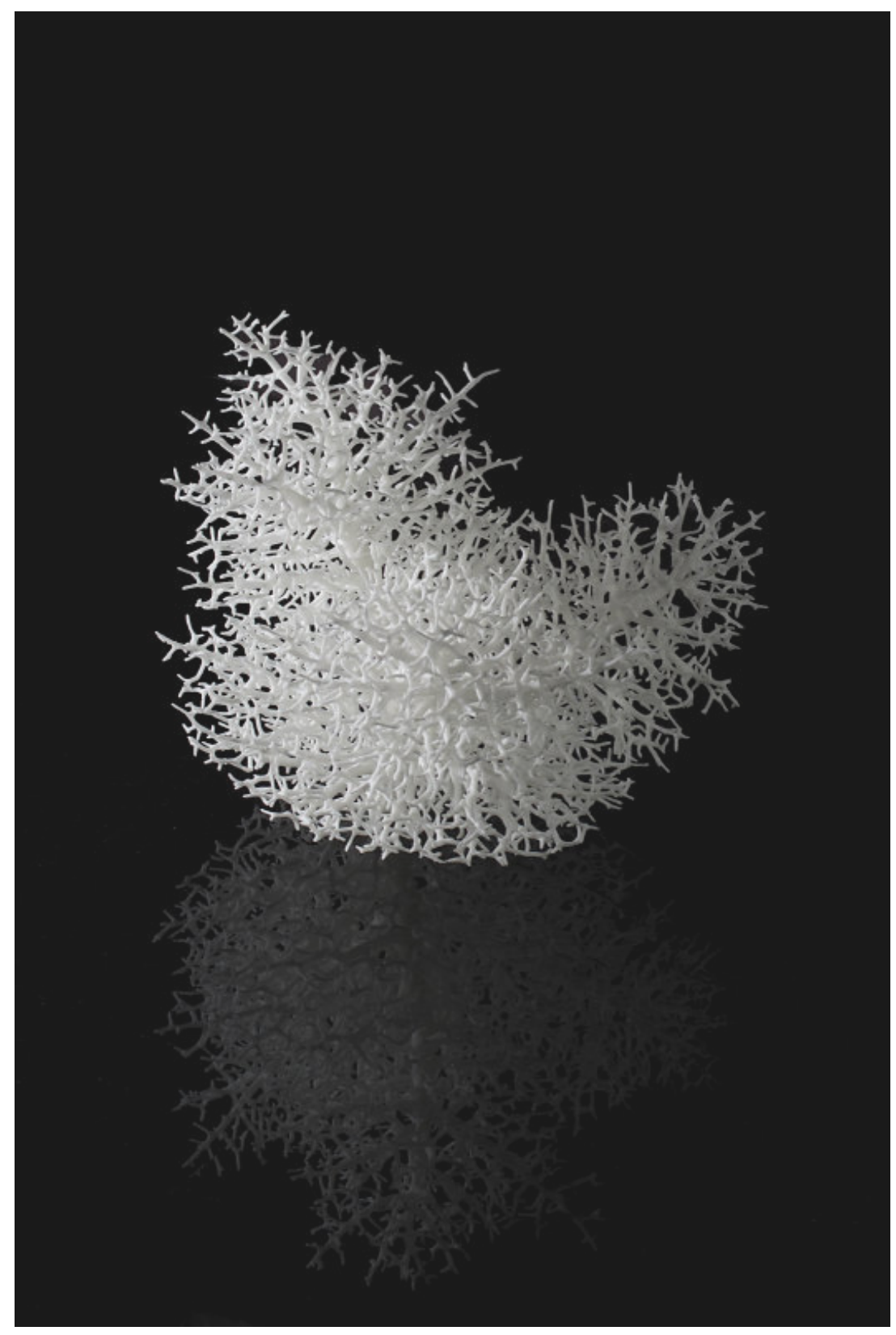


What makes computational design unique is the nature of mathematical thinking as the result of calculation skills of the computer, fig 14 is an example of generative growth rhythms applied to a branching tool, the result is an incredibly unique and intricate object. Concepts, ideas and aesthetics that were considered impossible to feasibly design not so long ago are now becoming a reality. The power of digital tools allows designers to push initial concepts and ideas past the limitations of their minds. However, one problem that has arisen from digital design is the uninformed and ignorant application of computational design aesthetics. Applying basic computational features and tools with no functional or meaningful reason, can in fact belittle the designs value.

This thesis aims to explore computational design aesthetics from not just an aesthetic point of view, but from a function driven approach, which in turn presents practical opportunities and a new approach to the design of golf equipment. 


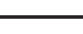

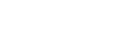


CHAPTER FOUR

LITERATURE REVIEW \& PROJECT REVIEW 


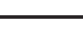

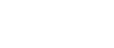


The selected literature addresses theories and methodologies specific to practise-based, exploratory research (research through design). This analysis interprets and tests the theories of Michela Turrin, Peter von Buelow and Rudi Stouffs to promote experimental design driven by digital technologies. 
Exploratory, performance driven parametric modelling

As discussed in the programme analysis, the combination of digital data tools and parametric modelling driven by the functional performance aspects of the putter is an innovative, performance driven exploration into the customisation of golf equipment. Therefore the question must be asked, how can parametric modelling effectively drive performance orientated design explorations?

Michela Turrin, Peter von Buelow and Rudi Stouffs (Turrin, Von Buelow, \& Stouffs, 2011) present a variety of ap plied and theoretical approaches to performance driven, parametric design explorations. Their collaborative paper; Design explorations of performance driven geometry in architectural design offers insights into the benefits of using parametric modelling to achieve a performance orientated process in design. Their specific focus is the benefits of these processes in architectural design, however the same underlying principles can be applied to the exploratory research in this thesis.

Their paper describes a wide range of computer aided conceptual design (CACD) methods used as both theoretical and applied design tools. Through analysis of these methods, the authors discuss the importance of initial design requirements, constraints and desired function.

One limitation however is they go on to state that "it is worth noting that the majority of these methods focus only on function of the design rather than its shape, and that most of them do not allow backtracking in the design process." 
As discussed in the programme analysis, depending on the nature and manner of parameters formulated, these limitations can be avoided through parametric modelling and further, be seen as potential opportunities for functional parameters.

The authors also mentions that: "by using performance orientated design, the level of interdisciplinarity, level of complexity, and the key impact that geometry has on the realisation of the performance related goals naturally increases." That is to say, through application of realworld performance orientated parameters, the quality, in terms of functional performance related characteristics of the output are improved.

An example of performance orientated design in this research is the application of functional characteristics of the putter, as discussed in the site analysis. Attributes such as loft and lie could be applied as singular parameters in a whole model, to be defined by an individual's preferences. This type of parameter promotes a custom fit and therefore it is assumed that performance will be improved. 


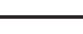

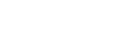


This project review analyses a variety of theoretical design concepts and professional design projects. They have been carefully selected based on their ability to provide insight into particular design tools and methods used in the exploration of research objectives. The specific case studies identified are unassociated with sports design, in an attempt to seek projects that push the limits of design tools and methods as identified in the programme analysis. 
Knotta Chandelier - Matter design. 2013.

Peroni picolla - Morgante, Andrea. 2013.

Brazil - Widrig, Daniel. 2009.

Structural joint - Arup. 2014.

Kinesis - Widrig, Daniel. 2011.
$1,2 \& 3$

\section{1}

$1 \& 3$

$1 \& 3$

$1,2 \& 3$ 


\section{RESEARCH OBJECTIVES}

This segment analyses existing case studies that provide insight into particular design tools and methods that are used to achieve the research aims and objectives of this thesis. The specific case studies identified are directly unassociated with sports design, in an attempt to seek projects that push the limits of design tools and methods identified in the programme analysis.

The principle objectives of this thesis are to develop a series of experimental design outputs that:

1. Integrate and synchronise emerging digital tools in the ideation phase of the design process

2. Utilize individual performance data as form defining inputs

3. Explore the potential of new aesthetics to enhance functional criteria

4. Preserve critical features of traditional golf club design. 
Fig. 15 | Knotta chandelier, Matter Design

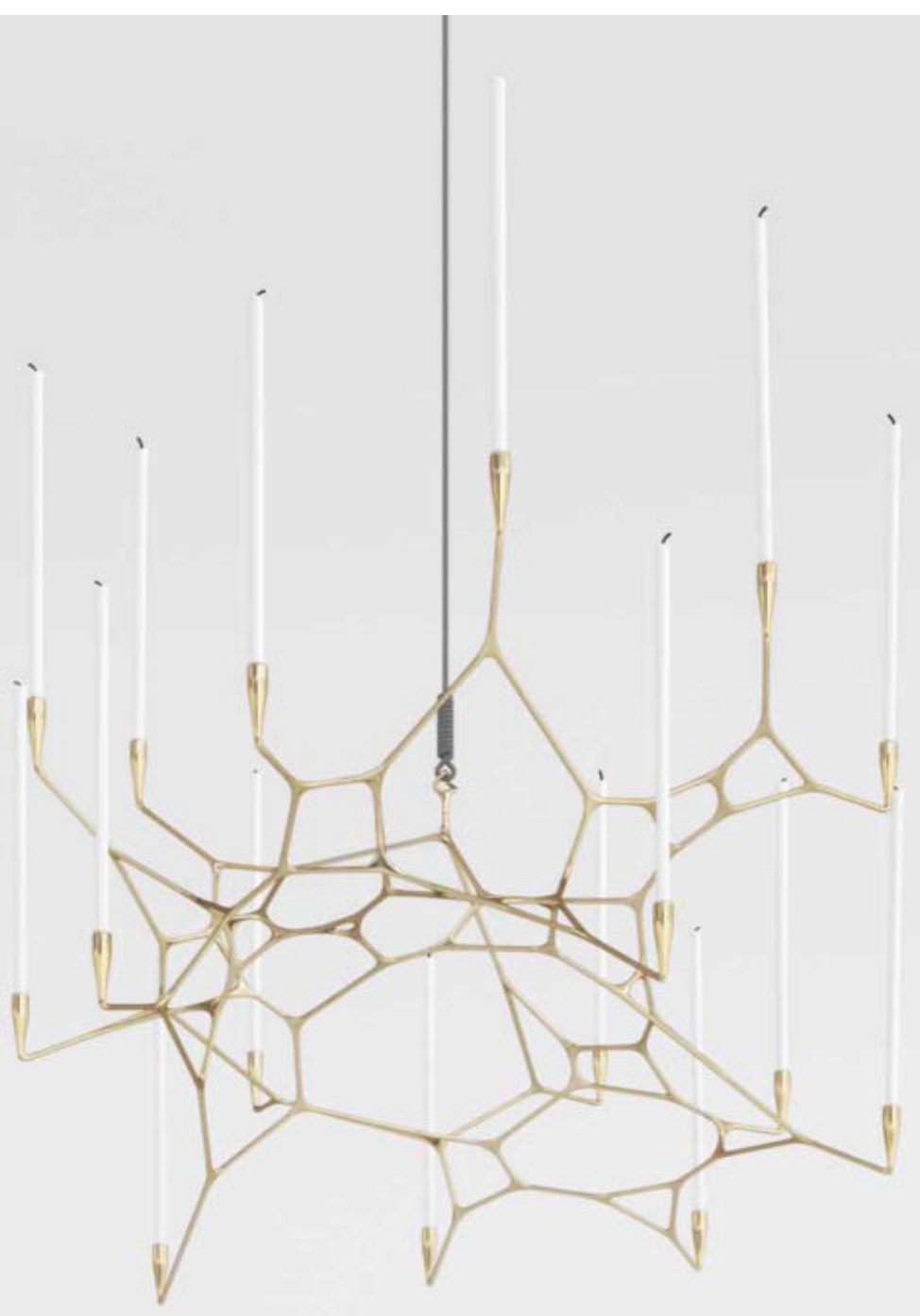




\title{
KNOTTA CHANDELIER, 2013.
}

\author{
MATTER DESIGN
}

Knotta (2013), by Matter Design is a brass chandelier that breaks traditional form of the radially symmetrical typology.

While the principles of symmetry aid in the construction of equilibrium to ensure a chandelier holds it's candles vertically, this constraint is no longer necessary in the digital era. (Matter, 2013).

Knotta Chandelier explores the idea of using digital, generative algorithms to produce a 3D model which adapts to changing inputs. Matter Design utilise digital software to develop two unique calculations. The first calculation generates "a dynamic relaxation of a string bundling, which produces a $3 \mathrm{D}$ branching network that serves to stabilise the system." The second "iterates through this dynamic relaxation re-determining the new center of gravity." The final output is said to be an "equilibrium" of the two calculations. The final object is first $3 \mathrm{D}$ printed, then cast in solid brass.

This case study explores the concept of adaptive, computational design aesthetic based on functional criteria. Matter Design test the traditional traits of product (chandelier) design through the application of digital tools. By utilizing features unique to digital tools, such as generative algorithms and optimisation techniques, Matter have created a adaptable relationship between digital and tangible. 
Fig. 16| 25.0 series by Andrea Morgante
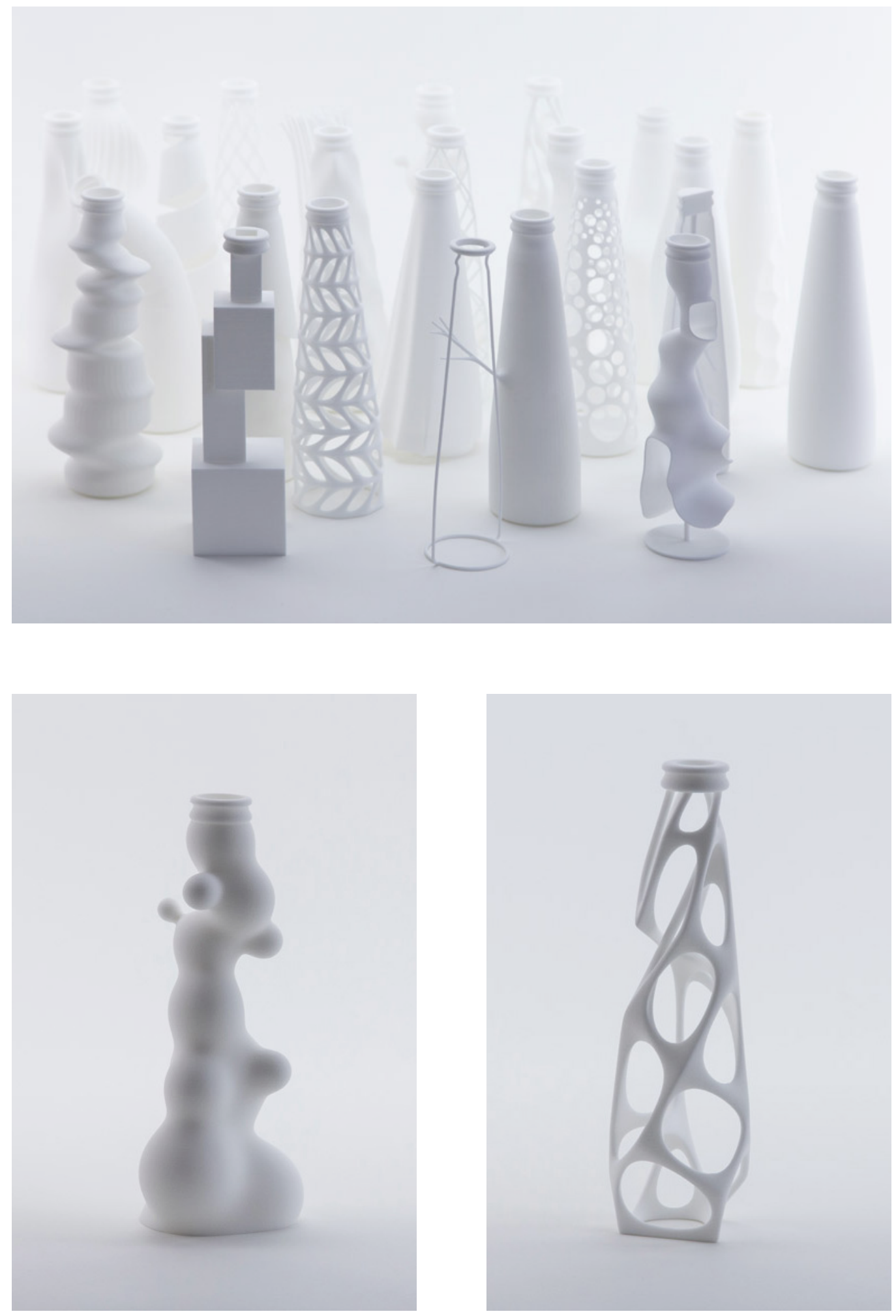


\section{$25.0,2013$.}

\section{ANDREA MORGANTE}

25.0 (2014), by Andrea Morgante of Shiro Studio is a installation displaying a collection of twenty-five individual 3D-printed objects, commissioned by M\&C Saatchi for Peroni Nastro Azzurro. The result is a subjective interpretation of Peroni's new "Piccola" $250 \mathrm{ml}$ bottle.

Each object is the result of a 'plastic mutation', where the original, intact shape of the Piccola bottle has been informed and digitally and genetically according to episodes relating to Italian design, architecture and Andrea's own memories growing up in Italy. (Shiro, 2014).

The 25.0 collection investigates the application of a range of digital sculpting methods on an existing geometry. The artistic approach, where every bottle "narrates a short story", has a more poetic nature about it, rather than a function driven approach. However, Morgante was able to effectively integrate a wide range of computational design aesthetics into the collection, resulting in a rich aesthetic exploration. Each of the 25 geometries were fabricated in a white polymer using $3 \mathrm{D}$ printing.

As seen in Fig. 16, this case study is a beautiful, comparative example of the breadth that digital tools offer. It pushes the boundaries of complex geometry and tests the limits of additive manufacturing through unique, delicate composition. 
Fig. 17| Brazil chair by Daniel Widrig

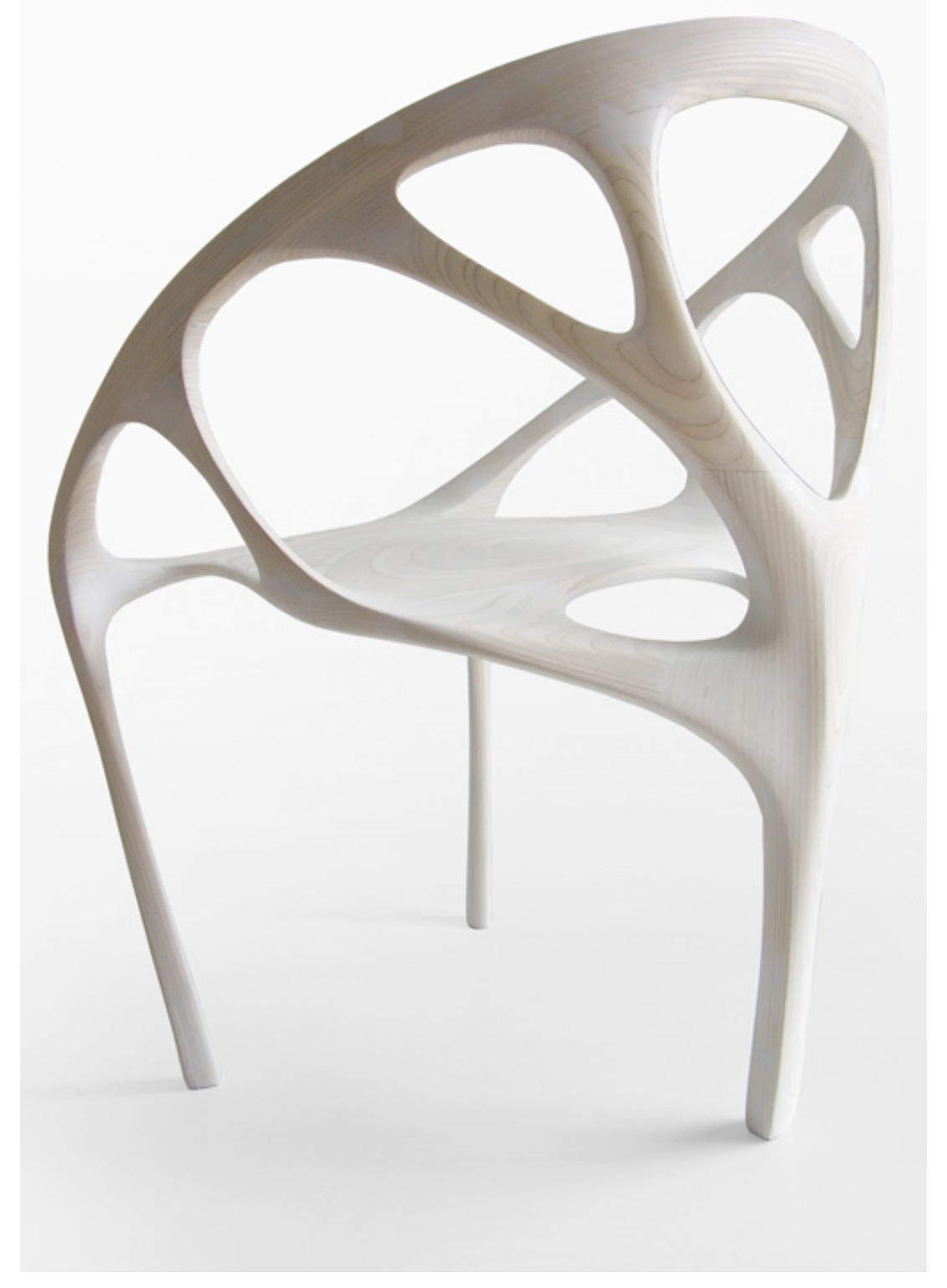




\section{BRAZIL, 2012.}

\section{DANIEL WIDRIG}

Brazil (2009), by Daniel Widrig is a limited-edition armchair built from laminated woods sheets via a 5-axis CNC router.

Featuring a structural bifurcation system, the chair has three legs that branch out from the floor and smoothly transform into armrests, a backrest and a seating surface in one fluid movement. (Widrig, 2009).

Brazil considers the synergy between CNC machining and digital optimisation tools. Widrig's three-legged chair is structurally and ergonomically optimised through digital dynamics simulation. The process allows for rapid, flexible design and development and "a straight-forward realisation of the complex geometry of the piece." Widrig has also used a 'digital-nesting' technique to split the chair into multiple components and cut them all at once from a single block of laminated wood, efficiently using limited resources.

This case study explores new opportunities in furniture design through the application of digital tools to change the perception of aesthetic function. The optimisation tools encourage the voronoi-esque aesthetic, which implies natural function. This idea has been further exploited through the singular, fluid bifurcation system. Ideas such as this are an important approach to utilizing digital tools and related aesthetics for new purpose. 
Fig. 18| Steel node iterations by Arup
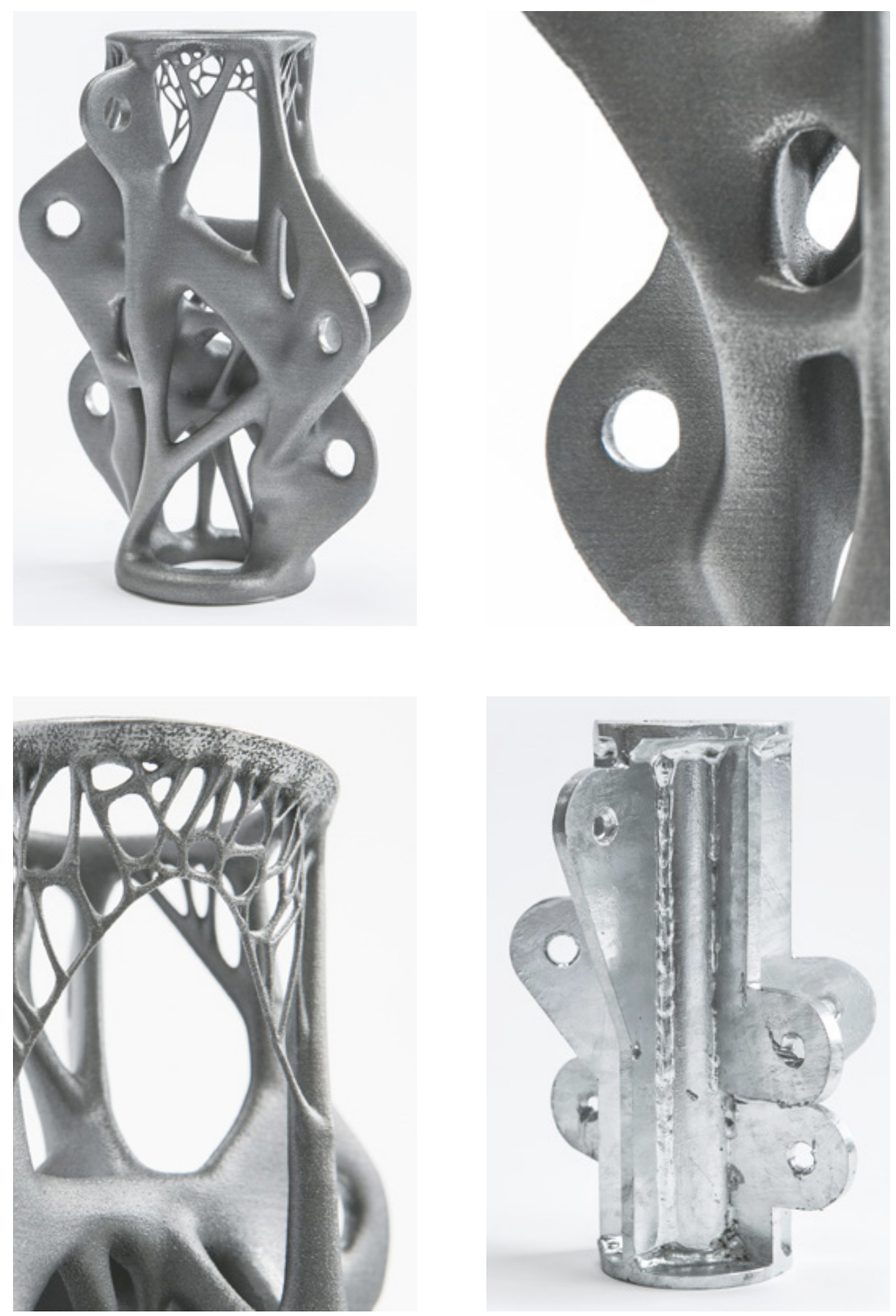


\title{
STEEL NODE, 2014.
}

\author{
ARUP
}

Steel node (2014), by Arup is a prototype 3D-printed steel construction join used to create more efficient structures.

This approach potentially enables a very sophisticated design, without the need to simplify the design in a later stage to lower costs. (Galjaard, 2014).

Arup's steel node explores a new design method for creating "critical structural steel elements" for tensile structures. They believe the approach is a completely new direction for both additive manufacturing, construction and engineering. The method is built around efficiency and optimisation, allowing the company to produce bespoke structural pieces while simultaneously minimising waste and costs. The node is still in preliminary materials testing stage, but Arup hopes to produce full size practical objects within a year.

This example questions traditional aesthetics and processes (fig 18 , bottom right) within architecture and engineering, pushing the boundaries of additive manufacturing and complex geometry (fig 18). The organic form is a direct result of digital mathematical calculation through optimisation techniques, a form that could not possibly be designed or manufactured without the use of digital tools. 
Fig. 19| Kinesis collection by Daniel Widrig
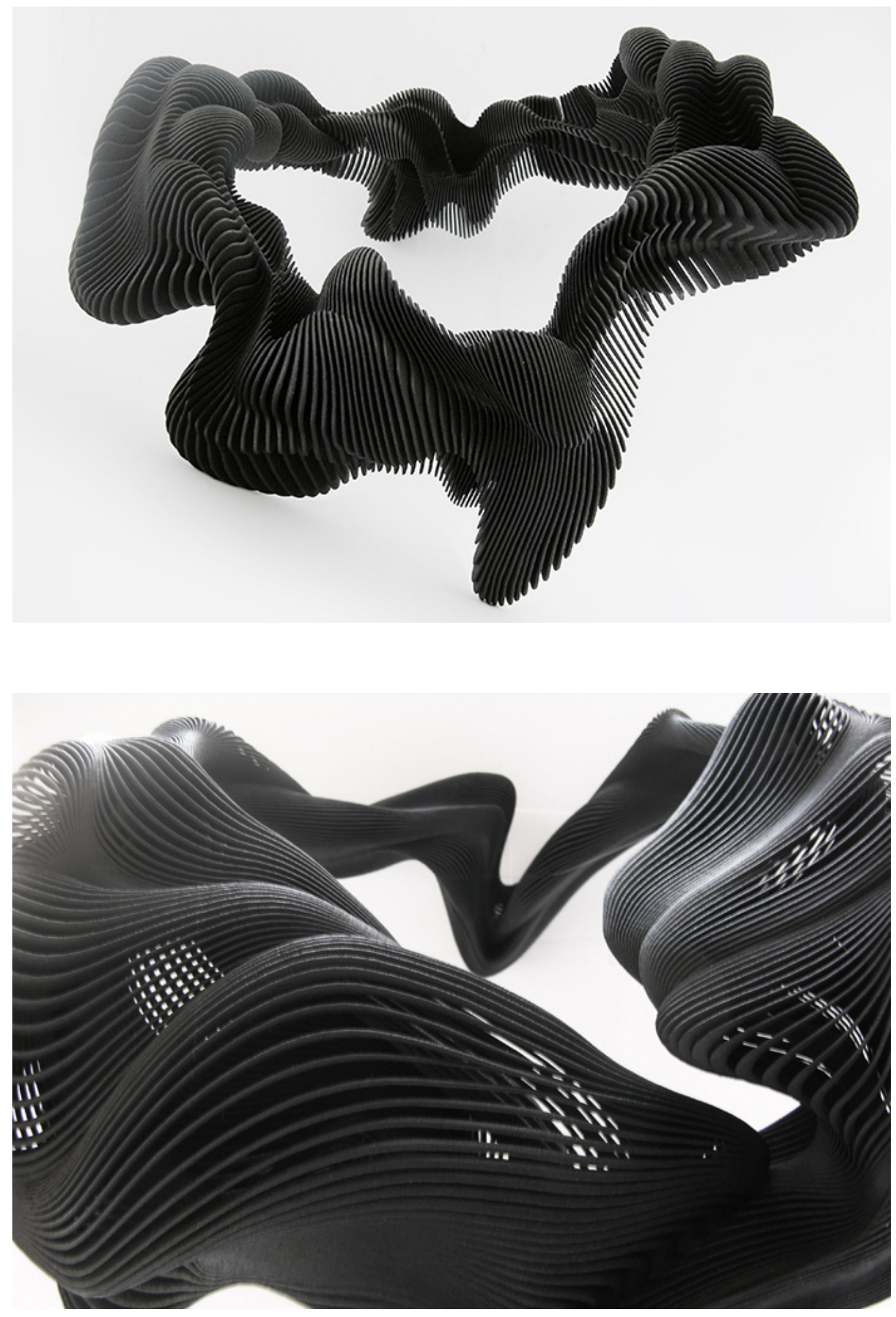


\title{
KINESIS, 2011.
}

\author{
DANIEL WIDRIG
}

Kinesis (2011), by Daniel Widrig is a collection of 3D-printed wearable sculptures, commissioned by MGX, Materialize.

We wanted to go a step further, and create customised objects that literally merge with the human body. (Widrig, 2013).

The Kinesis collection explores the concept of fabricating customised products based on a scan of the wearer's body, seeking the perfect fit. Widrig was able to analyse specific parts of the body through 3D scans, digital models, developing unique forms designed to "emphasize and exaggerate the body parts." The unique, individual, complex geometries were fabricated through a $3 \mathrm{D}$ printing specialist, using a selective laser sintering (SLS) process.

This case study explores new opportunities in product design through a unique combination of emerging technologies driven by individual inputs. Widrig pushes the boundaries of both customisation and traditional form in fashion design, through experimental application of contemporary digital tools, computational design aesthetics and additive manufacturing. Widrig is able to not only formulate complex forms as novel design solutions, but question traditional fashion design and pose new questions as to the potential aesthetic and function of a garment. 
Fig. 20| Visual map - computational design

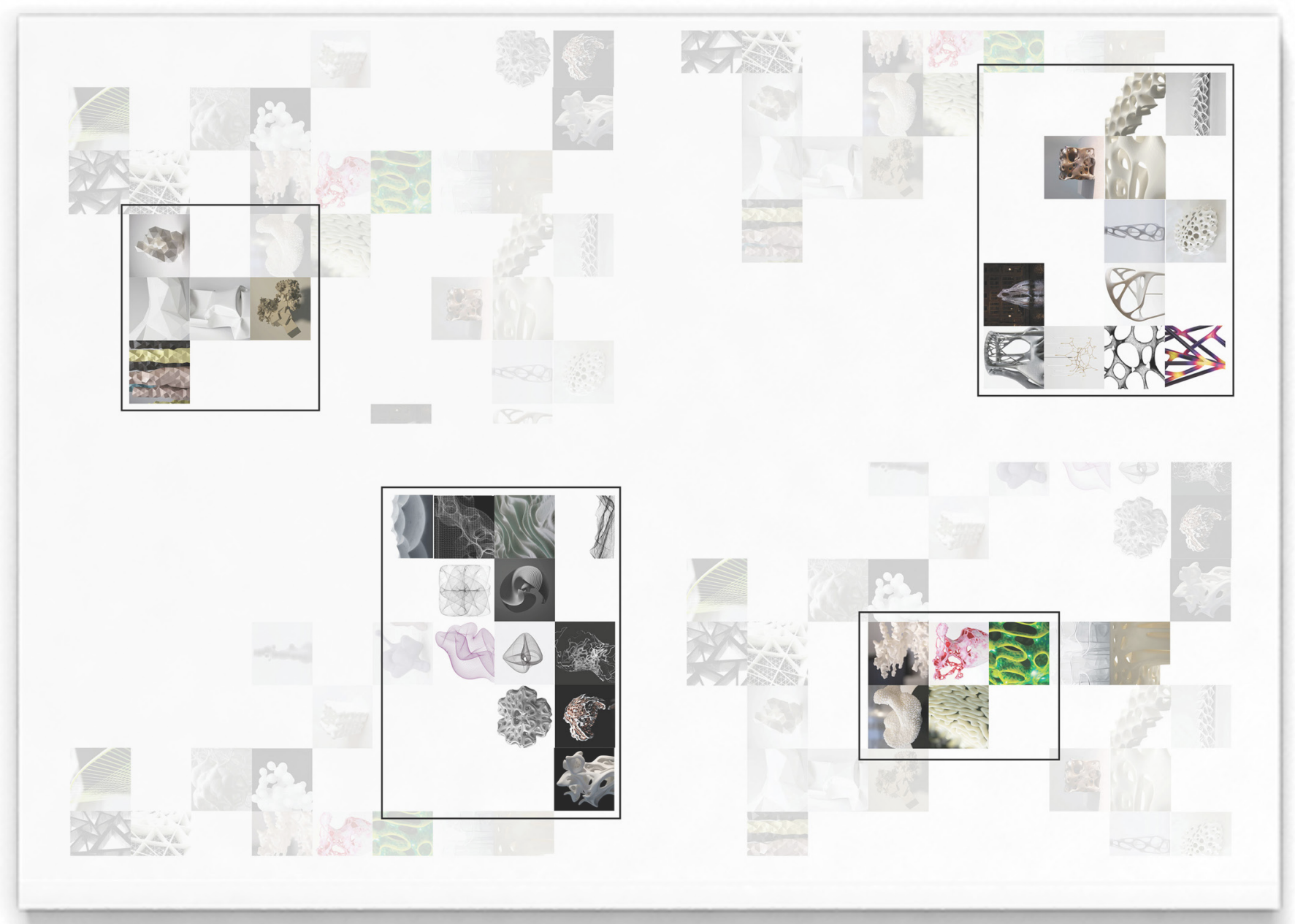




\section{VISUAL RESEARCH}

As discussed throughout the project review above, each case study offers a unique approach to utilizing digital tools in relation to various research objectives. Collectively, they showcase a range of computational design aesthetics realizable only through digital technologies.

In the next chapter, initial design experiments are conducted to test functional driven parameters in conjunction with computational aesthetics identified above. However the nature of digital tools, particularly those with parametric attributes suggest that an extensive range of aesthetic possibilities. Therefore it would be unjust to simply base the aesthetic exploration entirely on the findings above.

To explore a sufficient range of computational design aesthetics, a selection of visual research tools have been employed. Nobel \& Bestley (Noble \& Bestley, 2005) describe how visual research covers two main themes in relation to what might be termed 'analytical and propositional' methods: the visual deconstruction of existing work, and the development of new design strategies and methods.

In accordance with the case studies analysed above, thirty other examples, showcasing unique attributes to digital design have been selected, and categorised based on their individual aesthetics, style, and context. These images were laid out as a visual map (fig 20), that clearly identified visual trends within computational design. 
Fig. 21| Digital form guide

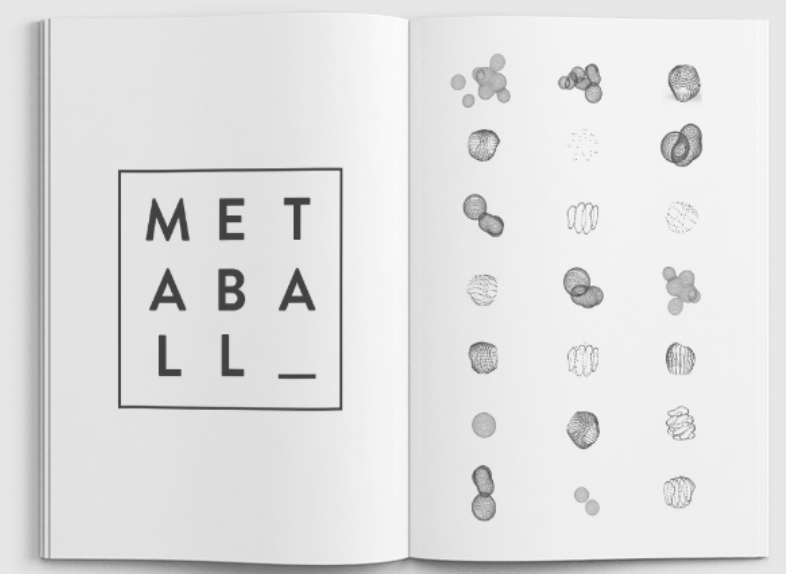


Visual mapping (Noble \& Bestley, 2005) helps to identify specific visual cues and styles and in this case six obvious trends within computational design. As there is no specific definition for each style, each group has been given a temporary title. Lattice, geometric, organic, curvaceous, voronoi and metaball. As exhibited in the project review, each category has its own potential to offer advantages in terms of functionality.

To better understand each group as a visual language and their potential for functional application, a booklet was created, the Digital Form Guide (fig. 21). The Form Guide not only visually communicates the deconstruction of each visual group, it was also created as a guide to making aesthetic based, functional choices throughout the experiment. 

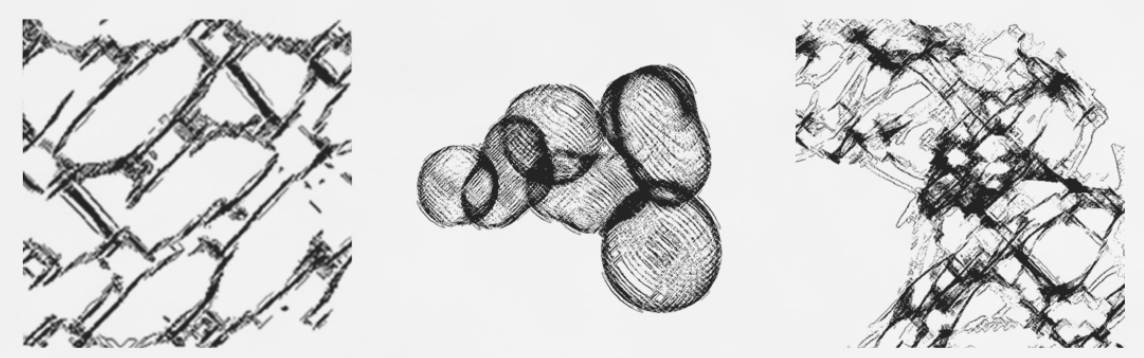

O这急

92 90000

000 20
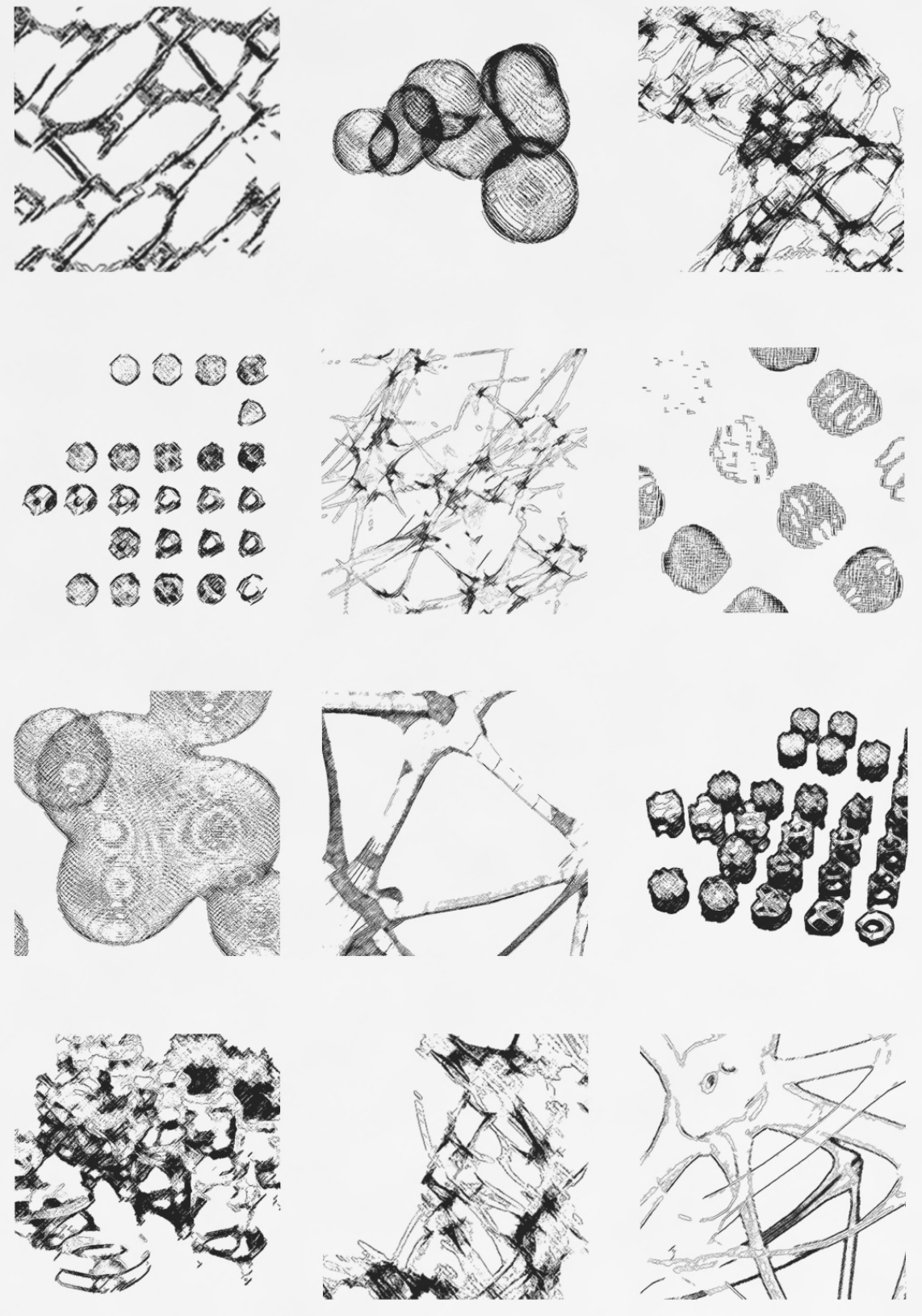
Fig. 22 exhibits a range of initial digital charcoal sketches by the author inspired by the examples in the project review and form guide. 


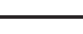

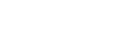




\section{CHAPTER FIVE}

\section{PRELIMINARY EXPERIMENTS}




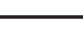

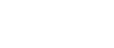




\section{FUNCTION VS. AESTHETICS}

This chapter exhibits a range of investigative design experiments inspired by case studies and theoretical design approaches described in chapter four. Each experiment probes a divergent feature exclusive to digital tools in relation to functional \& theoretical issues of club design addressed in chapter two. In a similar approach to the case studies in chapter four, this chapter seeks new opportunities through the application of computational design aesthetic as a result of digital tools. Traditional golf club design and shape are purposely disregarded throughout these experiments so that more open-minded approach is taken. However, even though overall form is disregarded, each experiment is still designed to consider both functional and aesthetic performance aspects. Designed using parametric modelling, these experiments are created to adapt and change effectively in relation to individual data. Individual experiments are laid out as an iterative series to aid in understanding the context, purpose and sequential process. 


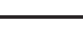

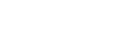




\section{SERIES ONE}

ATTRACTOR POINTS

$-79-$ 
Fig. 23 | Attractor points sketch

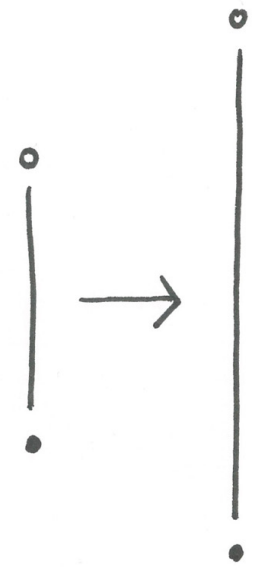

- POINT

- point aitractor

I DISTANCE

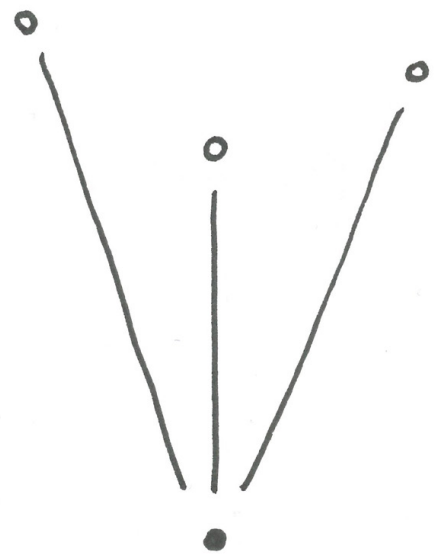




\section{ATTRACTORS}

at.trac.tor

$\mathrm{n}$.

1. Mathematics: A value or set of values toward which variables in a dynamic system tend to evolve.

The first series of experiments explores the technique of using attractor points in a parametric model. A point becomes an attractor point when a relationship is placed between it and another entity. In this case, distance is used to define the relationship. Therefore, when the coordinates of the point are adjusted, the distance is adjusted with it, giving a new distance output. The nature of parametric modelling enables further relationships to be implemented simultaneously (Fig 23). 

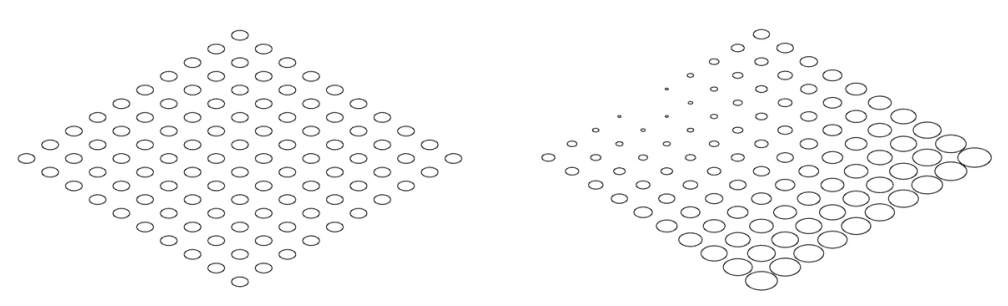

II

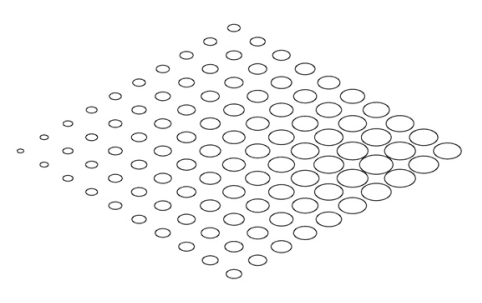

IV

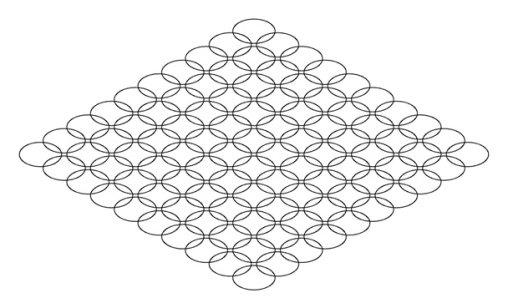

V
III

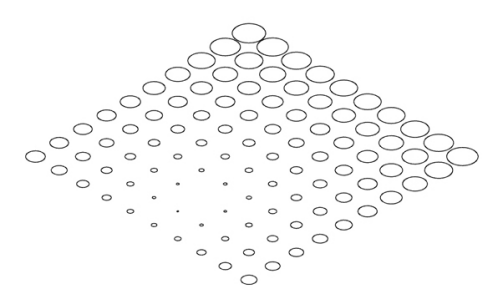

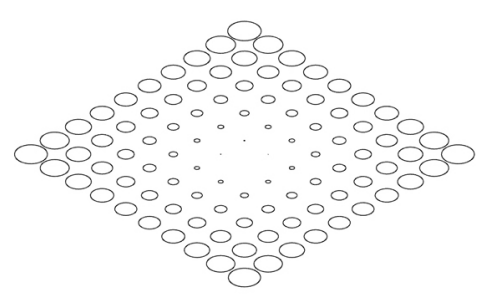

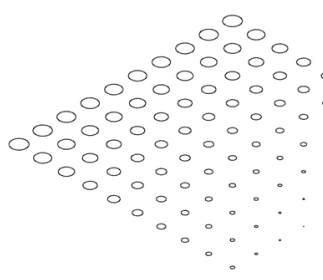

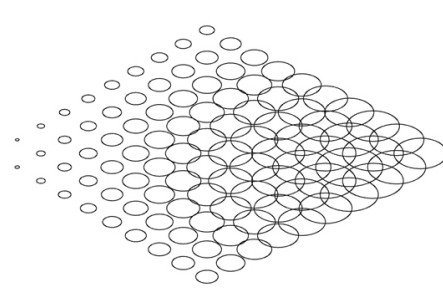

VI

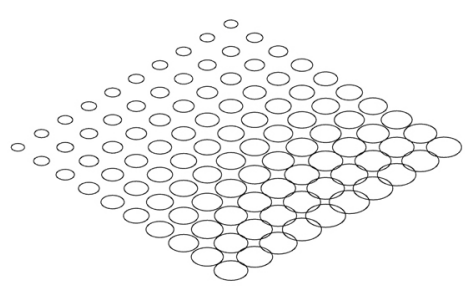




\section{ATTRACTOR POINTS 1}

The attractor points one series effectively demonstrates an example of these additional relationships. In this series, the relationship is defined between the first attraction point and separate $2 \mathrm{D}$ grid of points (rather than an individual point). The relationship is still defined by distance, but in this case the distance between the attractor point and each grid point individually, resulting in a new distance output for each point (fig 23).

The next parameter (relationship) was selected on its relevance to weight distribution. Circles were placed on each point of the $2 \mathrm{D}$ grid and a new relationship was established between the original distance outputs (between attractor and grid points) and the radius of each circle. Therefore, whenever the attractor point was moved about virtual space, not only would the distance output change but so would the radius size of the circle, this can be seen in iterations I, II, III and IV of fig 24.

However, it was discovered that without appropriate restrictions on the size minimum and maximum radius size, the circles would begin to overlap (iterations V \& VI). Restraints on minimum and maximum radius were added (VII, VIII \& IX). 

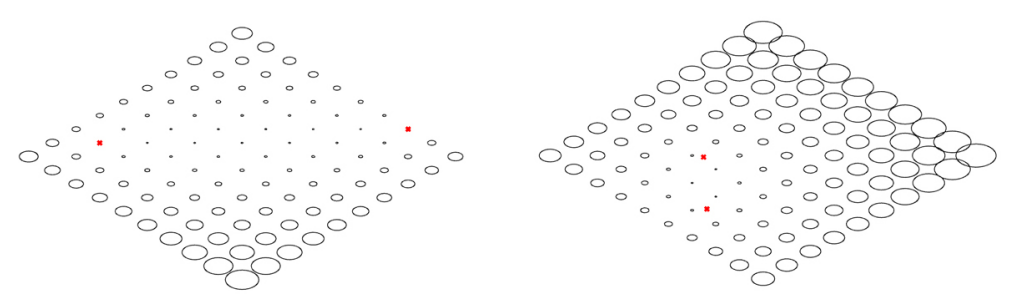

II

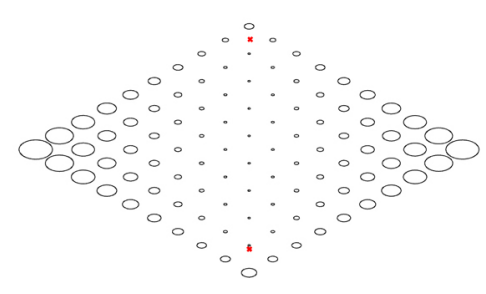

III

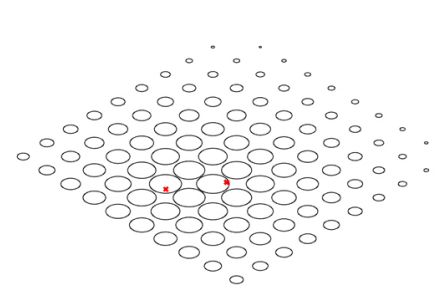

IV

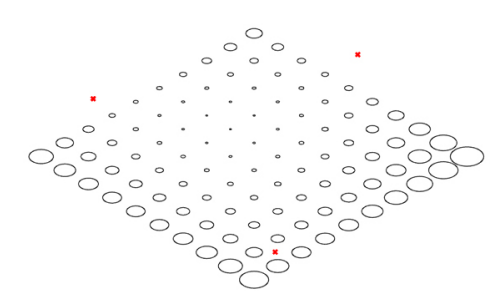

V

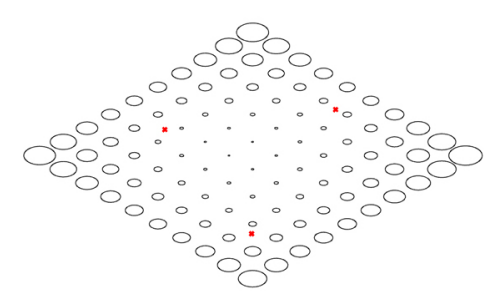

VI

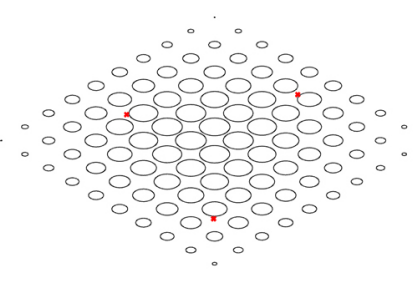

VII

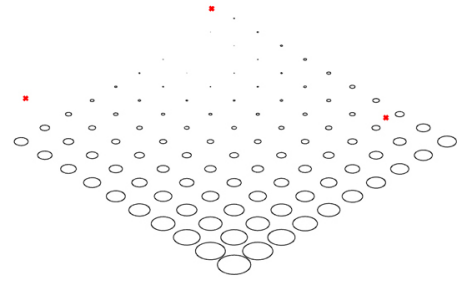

VIII

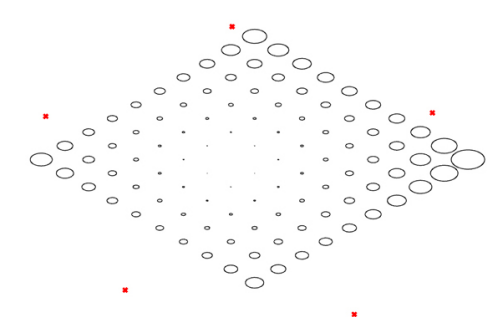

IX 


\section{ATTRACTOR POINTS 2}

The attractor points two series builds on, and refines the relationships employed in attractor points one. The first amendment was installing a second attraction point with the same genetics as the initial attractor point. The effect of having multiple attractor points is immediately obvious, as seen in iterations I, II \& IV of fig 25 (attractor points locations are highlighted in red). Having more than one attractor point allows for more complexity as across the grid, which in terms of weight distribution could be advantageous in relation to individual data inputs.

Iteration IV exhibits the ability to reverse the attractor data, as circle radius size increases as it nears the dots rather than decreases. Iterations $\mathrm{V}$ through to IX show variation using three, four and five attractor points. It has been noted that the more attractor points used, the less obvious the variation in radius size. 


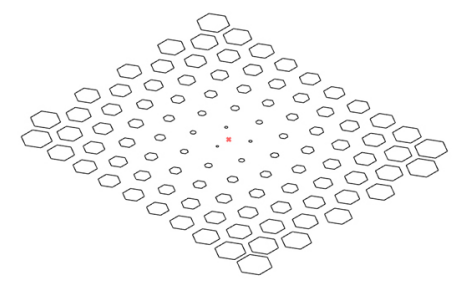

I

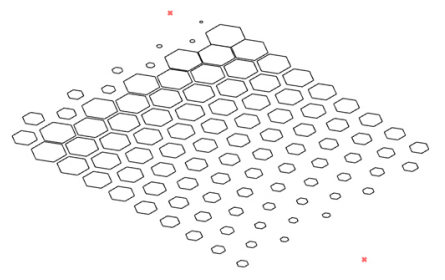

IV

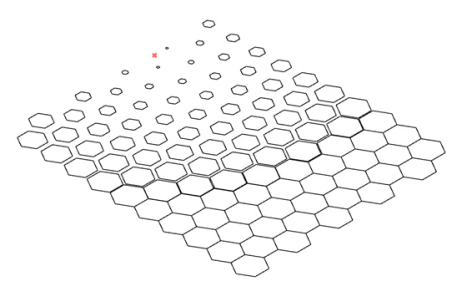

II

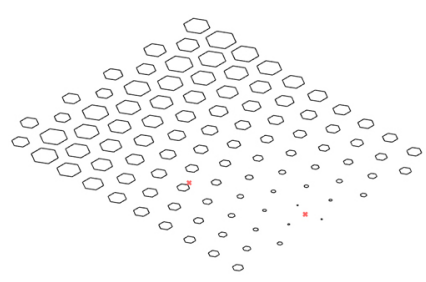

V

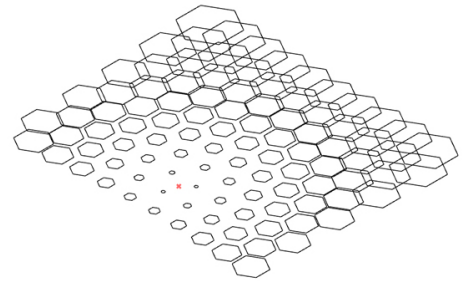

III

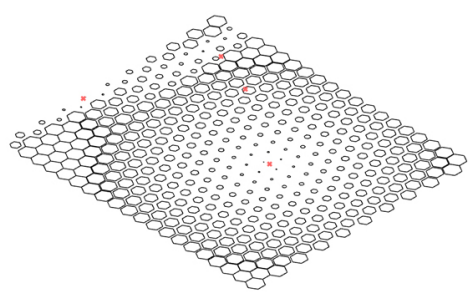

VI

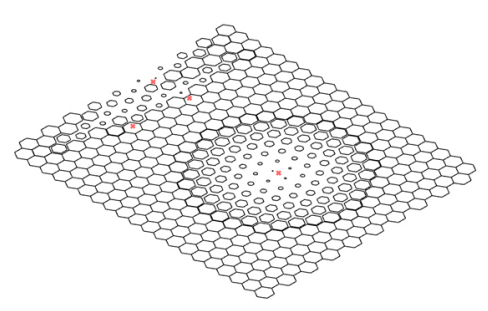

VII
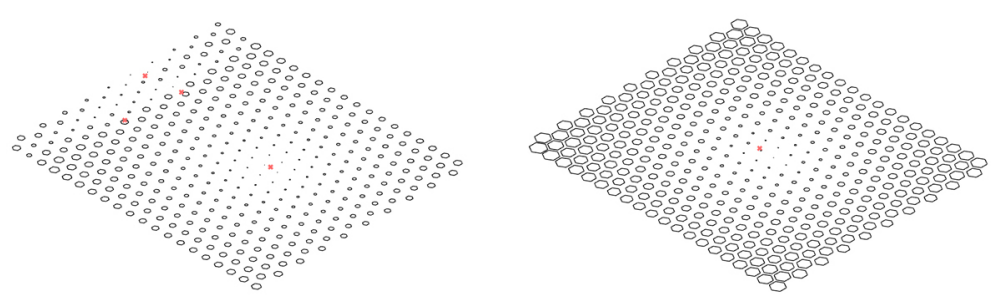

VIII
IX 


\section{ATTRACTOR POINTS 3}

The attractor points three series goes one step further and replaces the $2 \mathrm{D}$ grid of circles, with a grid of hexagons. The same relationships between attractor point and entity size were kept. Iteration I (fig 26) exhibit the use of single attractor point. However, due to the change geometry, as iteration III shows, new restriction had to be applied in terms of minimum and maximum size.

Iterations IV \& V exhibit the use of multiple attractor points, however it is noted that the order in which attractor points are assigned affects the quality of the relationship (seen in the contrast between IV \& V). In an attempt to increase visual complexity, Iterations VI through XI experiment with adjusting the number of hexagons, minimum and maximum values and the number of attractor points. 


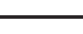

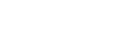




\section{SERIES TWO}

ATTRACTOR SURFACES

$-89-$ 
Fig. 27| Attractor surface sketch

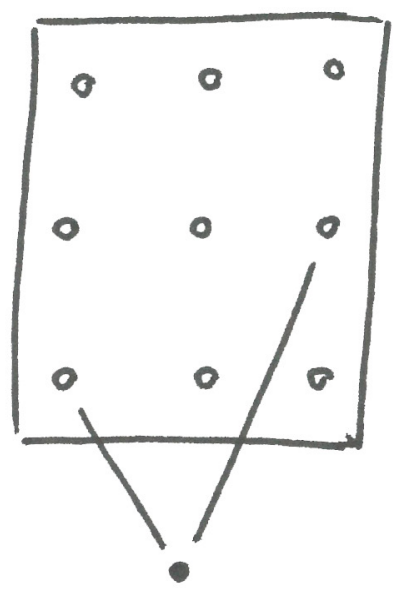

- POINT

- point aitractor

I Distance 


\section{ATTRACTOR SURFACES}

The second series of experiments also explores the technique of using attractor points, but rather than on a $2 \mathrm{D}$ plane, its is over a $3 \mathrm{D}$ surface. The attractor surfaces work on the same principles as the attractor points while keeping the same relations between designated points and distance outputs. One noticeable difference between the two series is the different methods needed to position (entity) centre points. With a $2 \mathrm{D}$ grid in series one, this is simply a matter of adjusting $\mathrm{X}$ and $\mathrm{Y}$ coordinates. When in comes to a $3 \mathrm{D}$ surface, this process must be done through UV mapping (process of mapping a 2D image on a $3 \mathrm{D}$ object). 


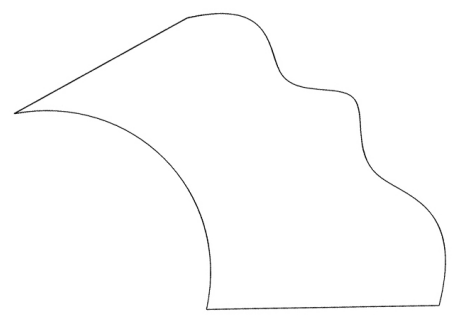

I

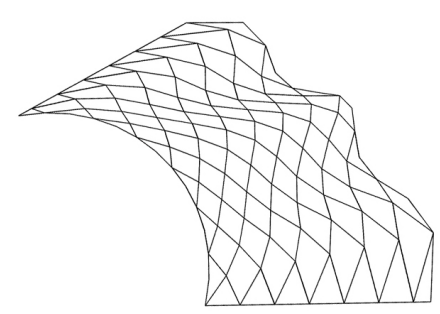

IV

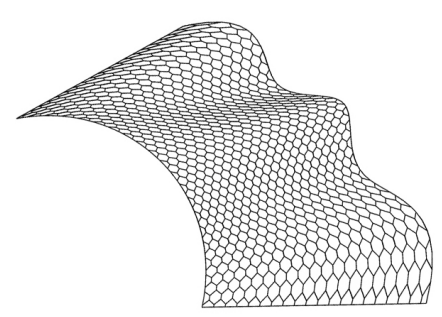

VII

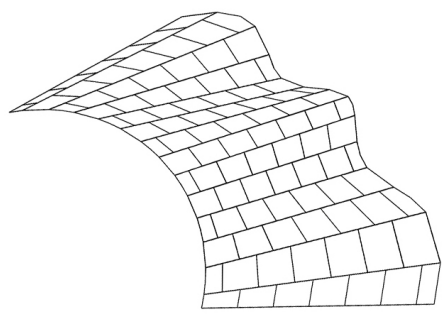

II

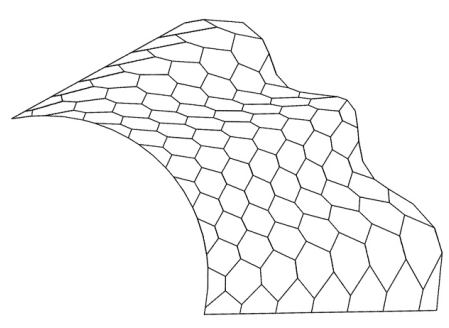

V

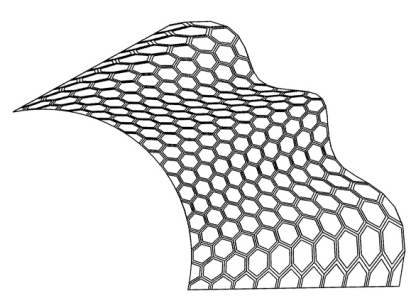

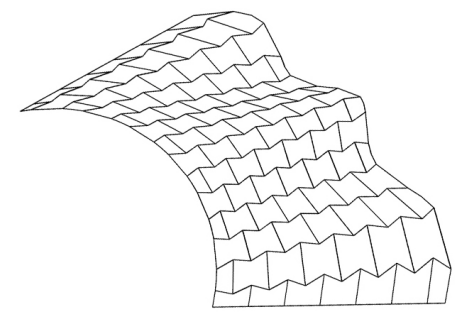

III

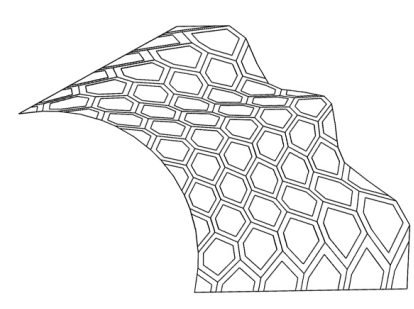

VI

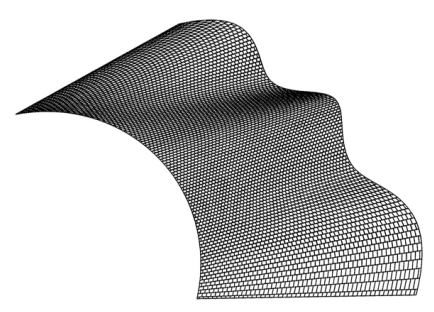

IX 


\section{ATTRACTOR SURFACE 1}

The attractor surface one series demonstrates a how UV mapping points across a free form surface (iteration I, fig 28) can bring a new dimension to attractor points. Rather than the simple, 2D shapes used in attractor points, these geometries take a 3D form that follows the mapped surface (iteration II). UV mapping divides a surface by a given number (panelling) while each division takes the form of a designated shape. One specific tool of interest, is the Hexagon Cell tool. It allows the overall output/shape of each panel to be determined by an number between 0-1, Iterations III, IV and V explore various form possibilities through its application.

Iterations VI through IX explore tools utilized in the attractor points series in combination with UV mapping tools. Iteration IV implements the scale tool, without the use of attractor points, while VII, VIII \& IX make adjustments to $\mathrm{U} \& \mathrm{~V}$ coordinates as well as the Hexagon cell tool. 
Fig. 29| Attractor surface sequence two

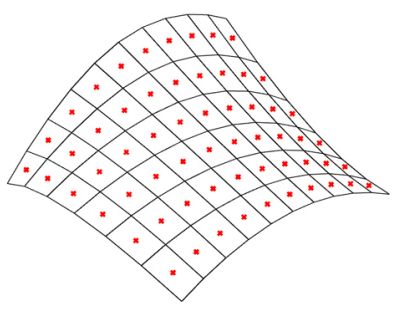

I

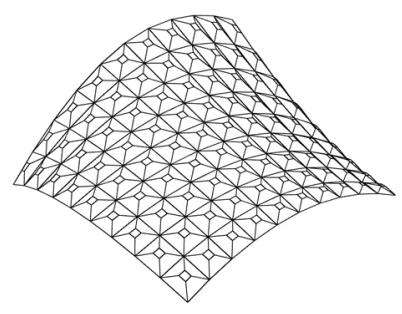

IV

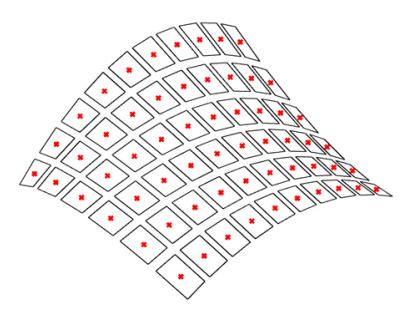

II

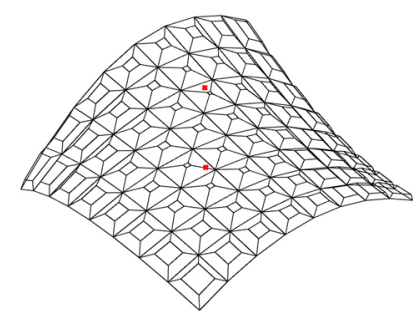

V

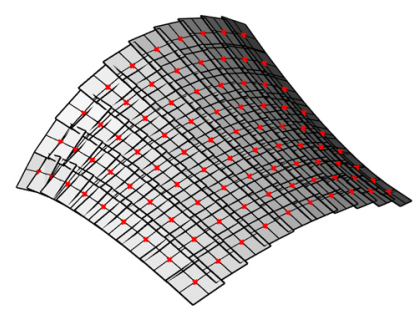

III

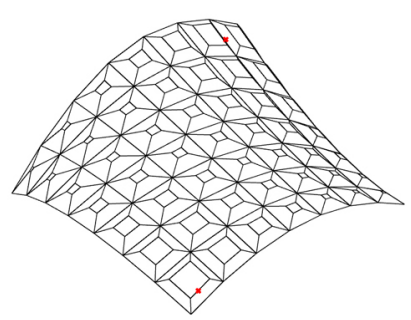

VI
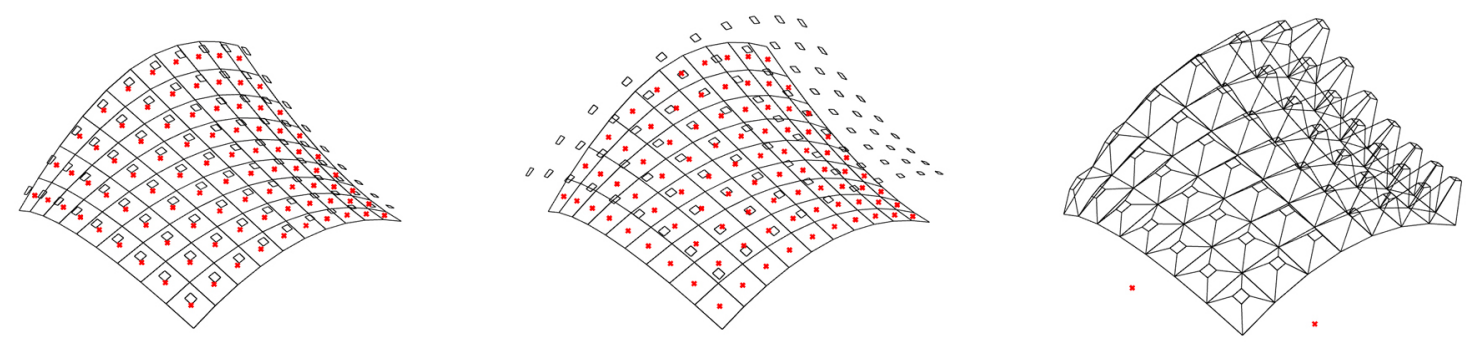

VII 


\section{ATTRACTOR SURFACE 2}

The attraction surface two (fig 29) sequence builds on, and refines relationships and tools explored from previous experiments. The first approach was to consider how these experiments could be more effectively applied in regards to weight distribution. This encouraged a push toward a more, 3D model, where weight could become a real factor. Iterations I \& II use UV mapping tools to divide the surface and scale the surface.

Iteration III exhibits the first failed attempt to make a loft between the original division and the scaled division while Iteration IV successfully completes a loft. Attractor points are applied in iterations $V$ and VI to adjust the scaled surface division while simultaneously continuing to loft. To further push the $3 \mathrm{D}$ factor further, the scaled surface division is offset from its original surface, shaped by point attractor's and lofted simultaneously, as shown in iterations VII, VIII \& IX. 
Fig. 30| Attractor surface sequence three

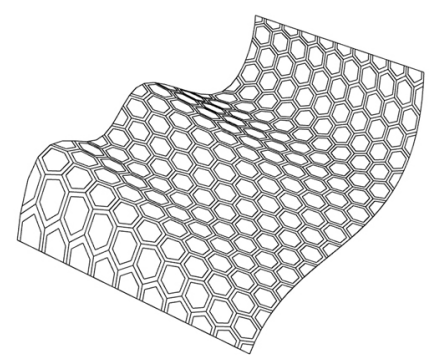

I

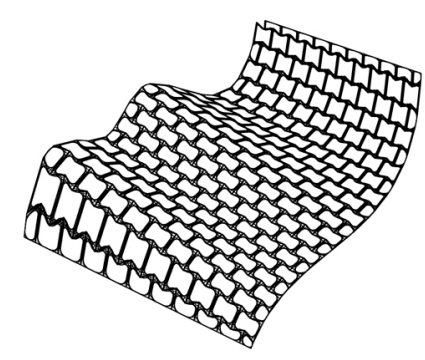

IV

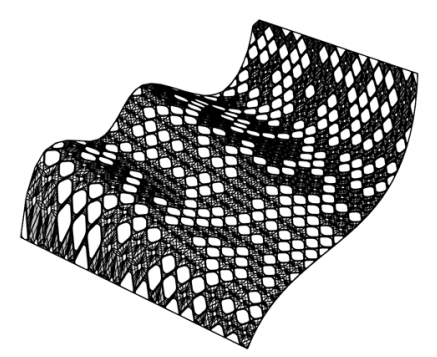

VII

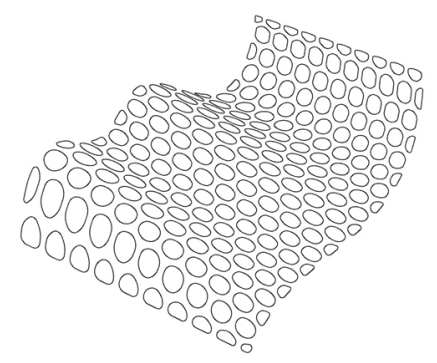

II

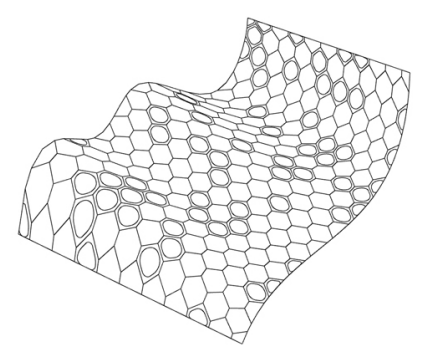

V
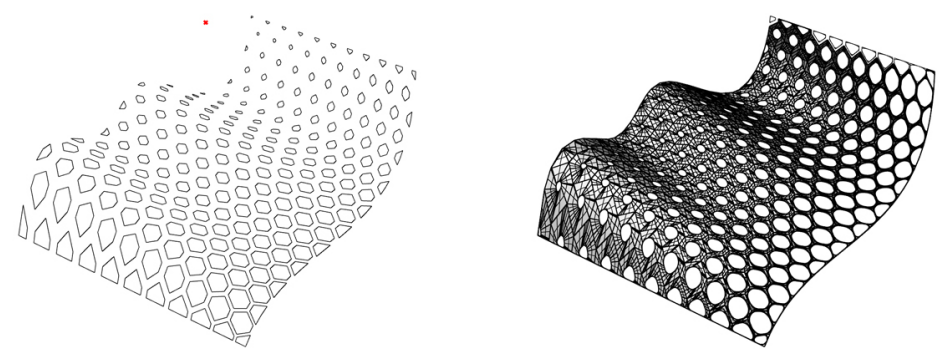

VIII

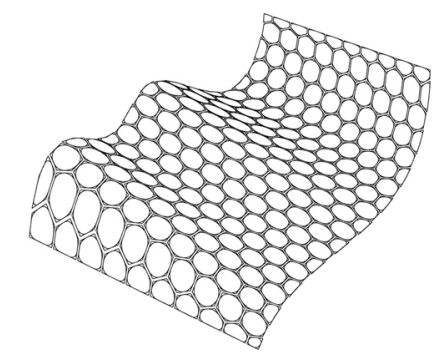

III 


\section{ATTRACTOR SURFACE 3}

The attraction surface three (fig 30) sequence explores further possibilities as discovered in the attraction surface two sequence. This particular experiment looks less at the functional aspects addressed in the previous two sequences and more at the aesthetic opportunities these tools can offer. Drawing on computational design aesthetics from the project review and digital form guide, these experiments aim to add more visual complexity to the functional parameters.

Iterations I through IV explore surface division shapes, loft thicknesses and densities. While V, VI \& VII test the idea of only specific functional data remaining visible, this type visual cue could enhance mental performance aspects. Finally, iterations VIII \& IX explore a combination of densities and scaled surface divisions driven by point attractor's. 


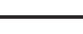

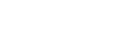


SERIES THREE

VORONOI MESHES 
Fig. 31| Voronoi patterning with attractor points

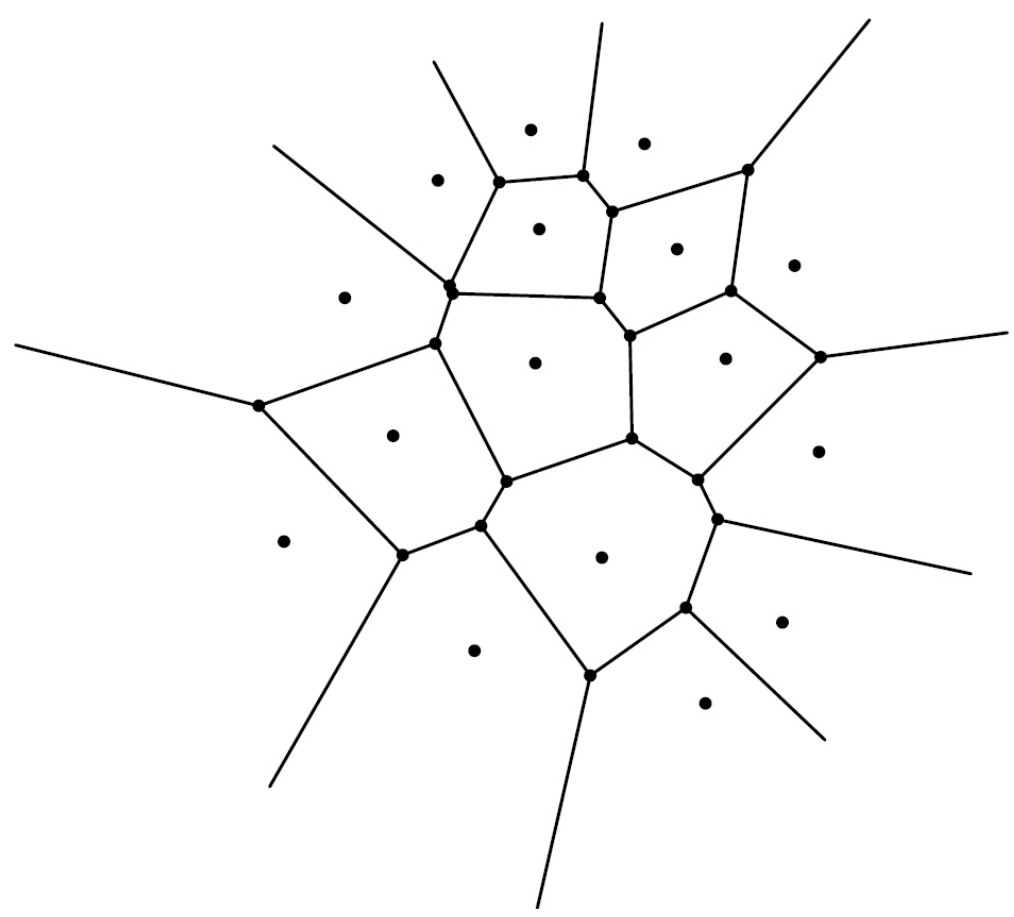




\section{VORONOI}

The third series of experiments looks to explore more natural forms, inspired by the voronoi, organic and curvaceous categories in the form guide. Although they have been categorised separately in the form guide it is noted that many of the organic and curvaceous examples from visual research are subsets of a Voronoi Diagram (fig 31).

The partitioning of a plane with a defined number of points into convex polygons such that each polygon contains exactly one generating point and every point in a given polygon is closer to its generating point than to any other (Weisstein, 1999).

The mathematics behind Voronoi diagrams are inspired by organic patterns therefore their application offers a very unique form seen in nature. Finally, this series aims to move beyond free form modelling surfaces and into virtual tangible objects (meshes). Meshes apply coordinates to virtual objects that can then be exported for physical manufacturing. 


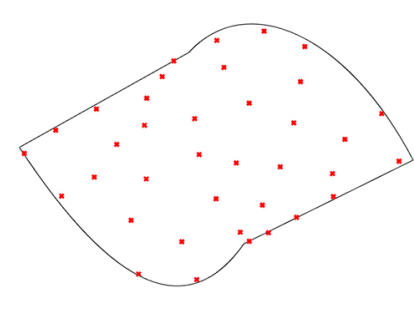

I

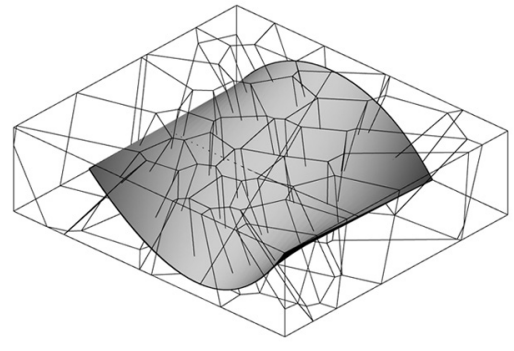

II

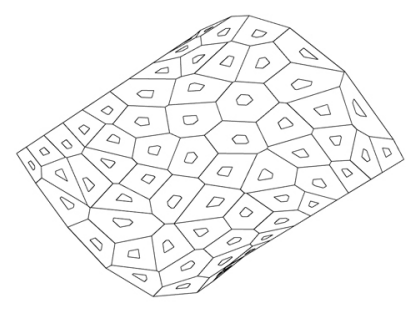

V

IV

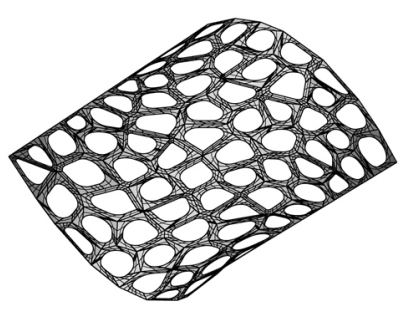

VII

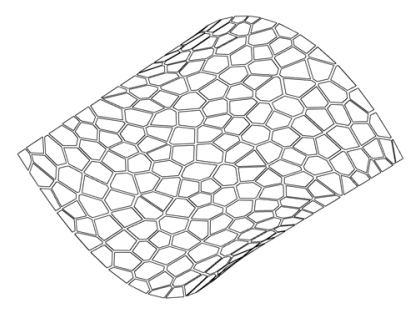

VIII

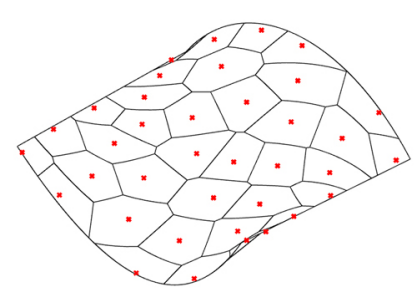

III

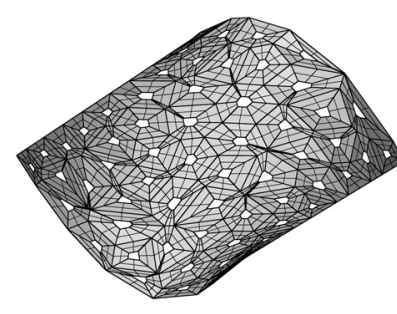

VI

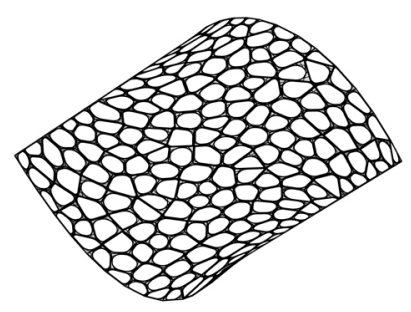

IX 


\section{VORONOI MESH 1}

The voronoi mesh one (fig 32) sequence exhibits the initial exploration into natural, voronoi based aesthetics. Rather than using attractor points, which may affect the mathematical calculation behind voronoi patterning, a random population of points has been implemented across the surface (iteration I). A voronoi feature was applied (II) to divide the surface based on this calculation (III). Iterations IV, V \& VI explore different visual languages through scaling and lofting. Iterations VII, VIII \& IX take these variations one step further by applying an organic rounding technique to the calculated geometry. 
Fig. 33| Voronoi mesh sequence two

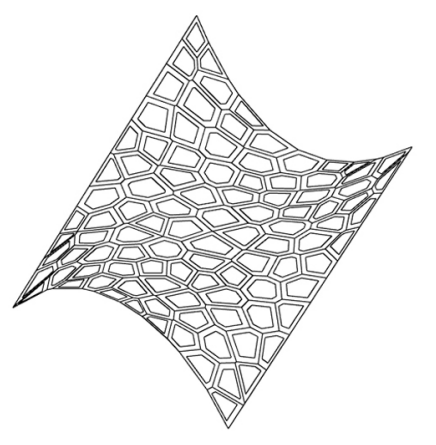

I

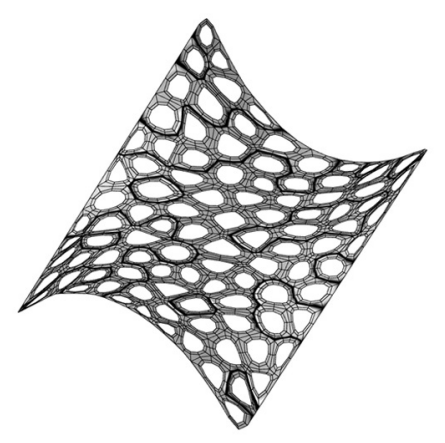

IV

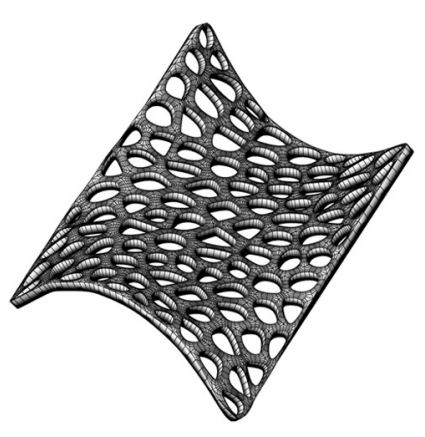

VII

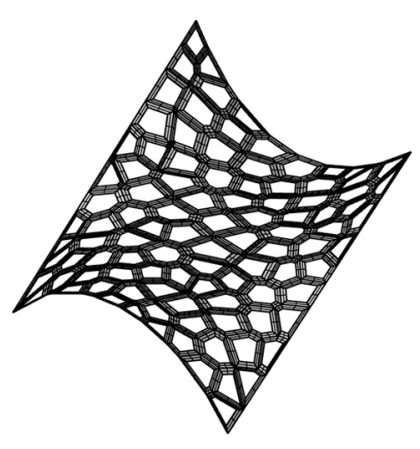

II

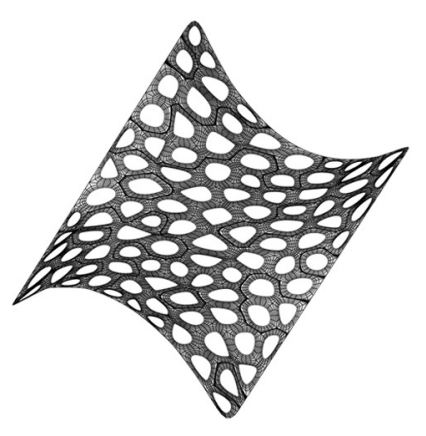

$\mathrm{V}$

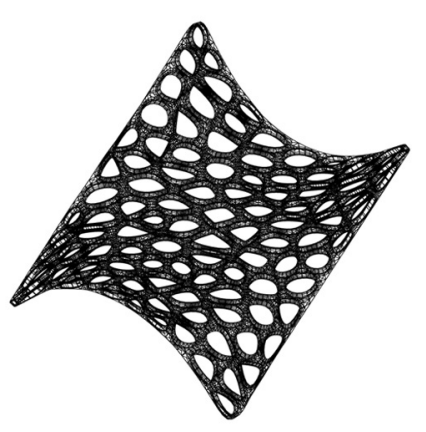

VIII

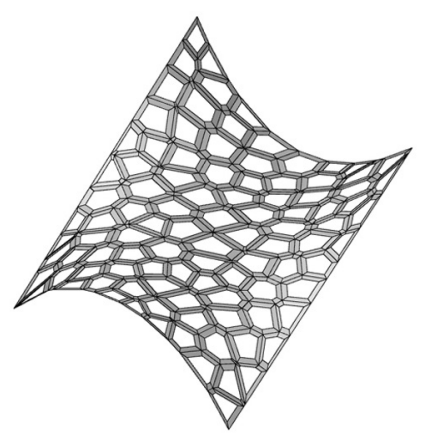

III

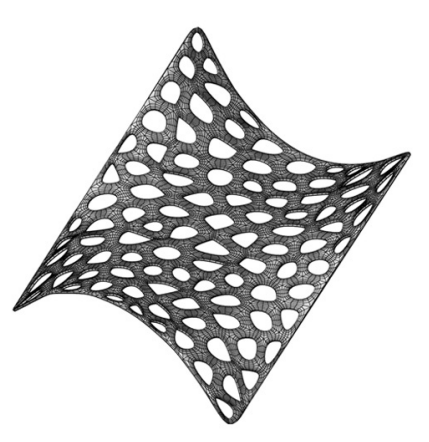

VI

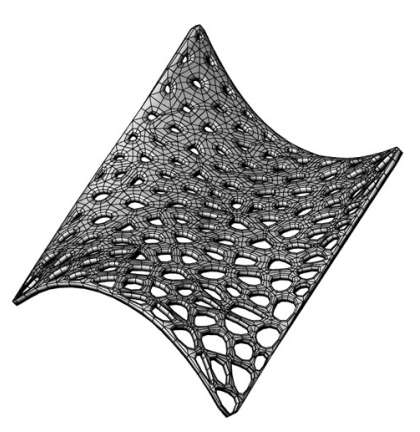

IX 


\section{VORONOI MESH 2}

The voronoi mesh two (fig 33) sequence focuses specifically on created a $3 \mathrm{D}$ mesh using aesthetics extracted from the voronoi mesh two sequence. Iterations I through $\mathrm{V}$ test visual complexity and organic aesthetic through adjustments in density, lofting options, rounding techniques and selective geometry. A powerful tool is then used to transform surfaces into a collection of vertices and polygons, otherwise known as a mesh (VI). The visual adjustment is not obvious until a thickening tool is applied to the mesh as seen in iterations VII \& VIII. These objects can now be exported for physical manufacturing. Iteration IX is an example where point attractor's are implemented to control the distribution of mass. 


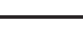

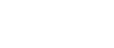


SERIES FOUR

3D ATTRACTORS

$-107-$ 
Fig. 34| 3D attractor sketch

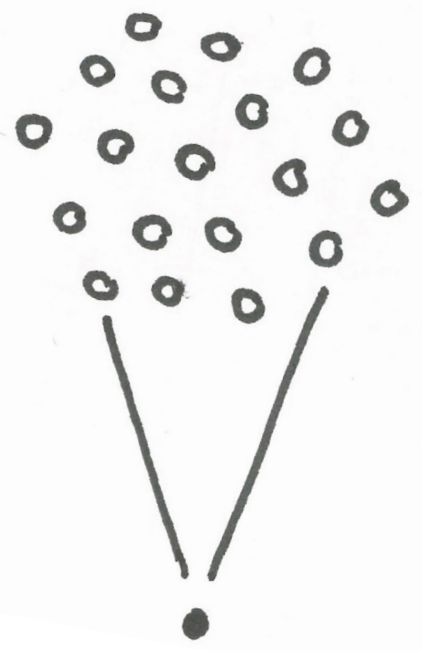

- POINT

- point aitractor

I Distance 


\section{D ATTRACTORS}

The $3 \mathrm{D}$ attractor series follows the same principles and ideas as series one; attractor points. However, two major changes are made throughout the series. The first sequence, 3D attractor's one applies a 3D geometry to each point, rather than the two-dimensional (2D) curves seen in series one. The second sequence $3 \mathrm{D}$ attractor's two take this idea one step further by applying geometries to a $3 \mathrm{D}$ cube of even points, rather than a single plane. The driving aim behind series four is to create a point attractor based 3D geometry which could be subtracted from separate base geometry to provide that base with a functional distribution of weight. Essentially designing the amount to be taken away. 
Fig. 35 | 3D attractors sequence one
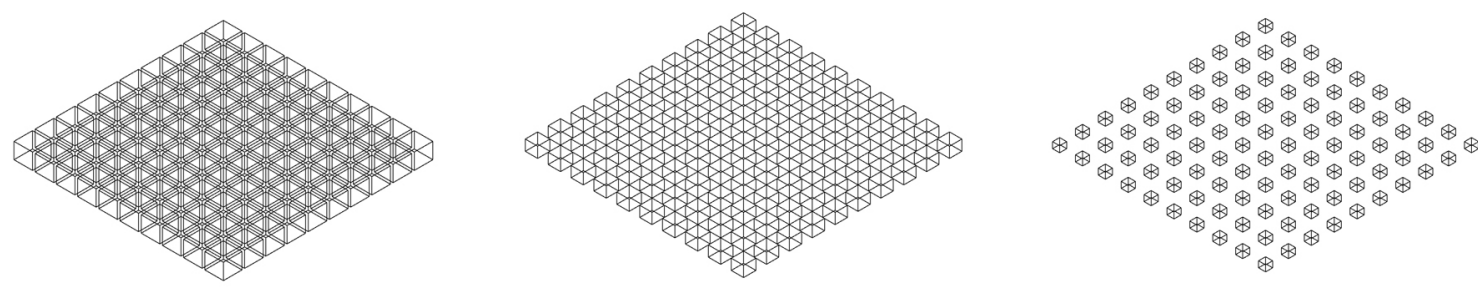

II
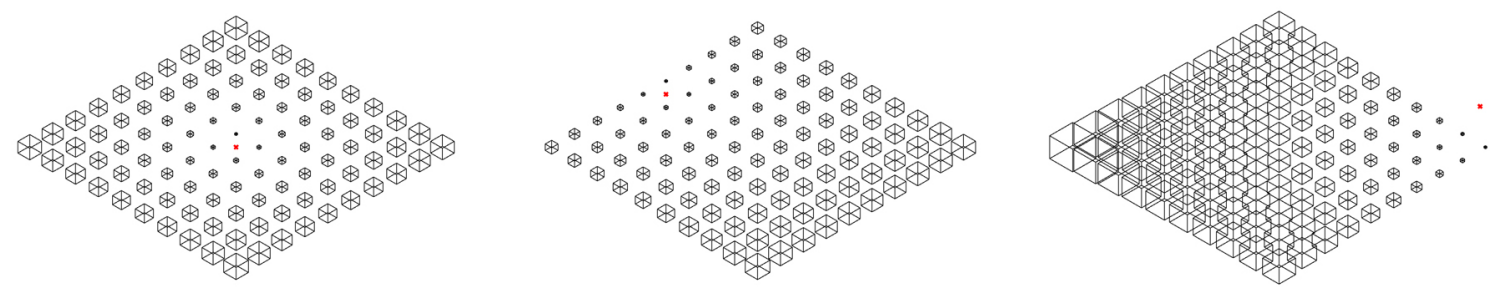

IV
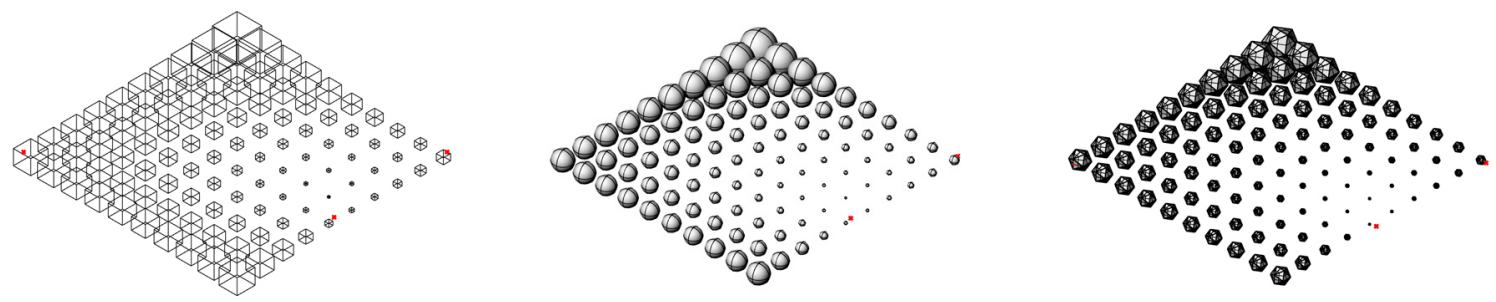


\section{D ATTRACTORS 1}

The 3D attractor's one (fig 35) sequence explores the use of attractor points to define scaling of $3 \mathrm{D}$ geometry. Iterations I, II III exhibit the scaling of geometry based on a $2 \mathrm{D}$ grid as centre-points, while IV through VII display the same scaling of geometry, however the scaling is determined between the centre point and the designated attractor point. Iterations I through VII explore these techniques using a simple cube as the defined geometry, iterations VIII \& IX explore the same techniques using a sphere and a dodecahedron $(12$ sided polyhedron). 

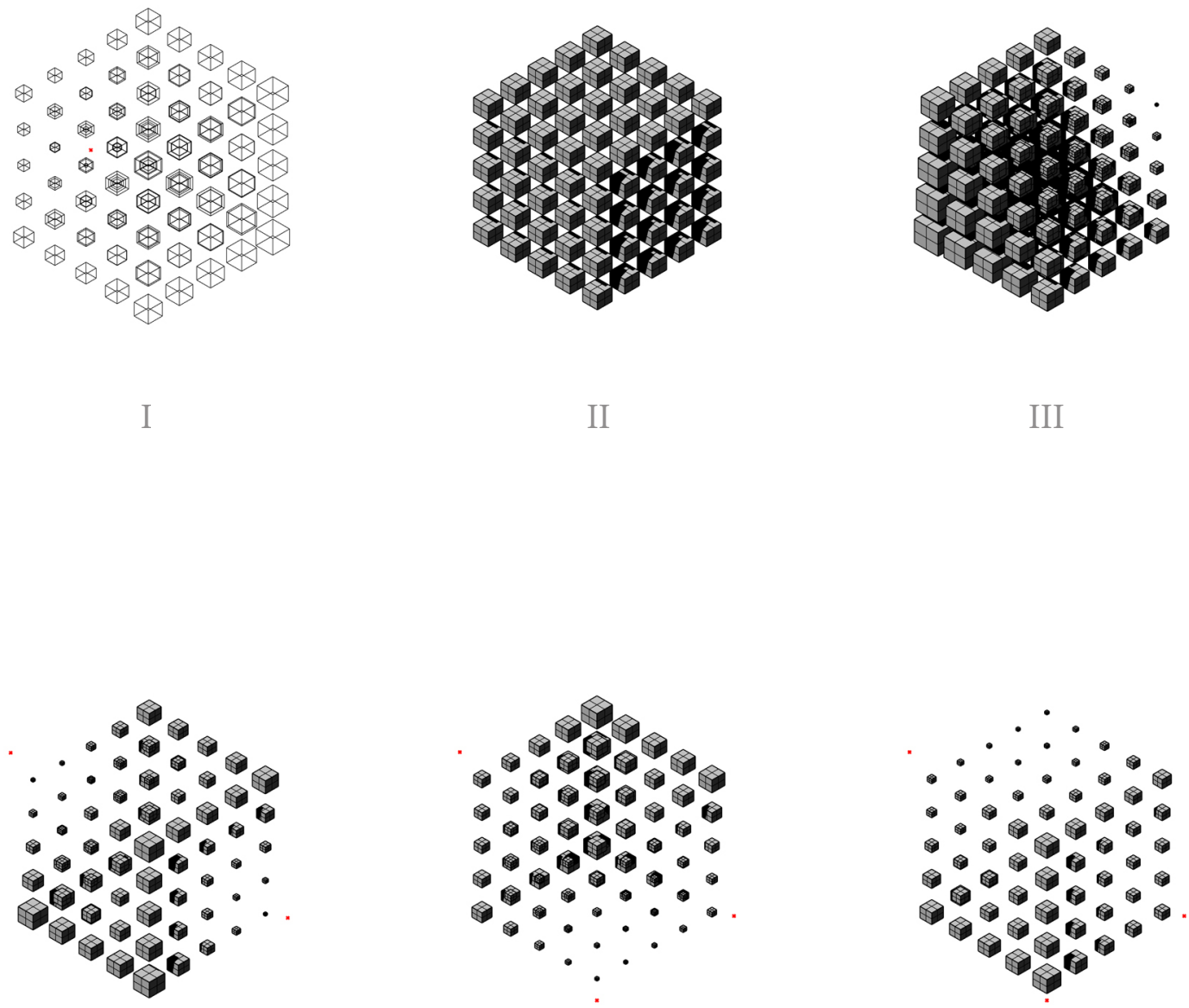

IV

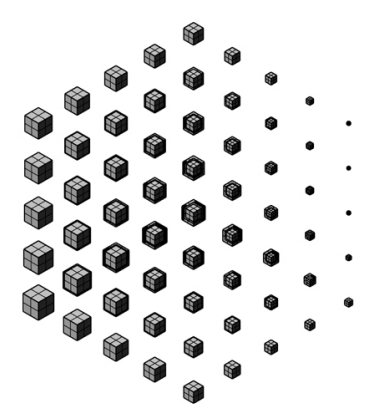

VII

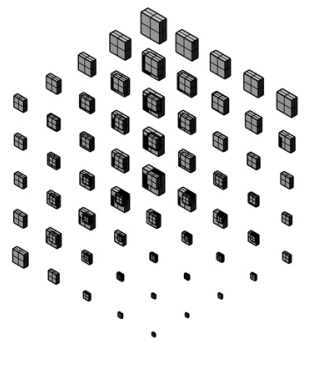




\section{D ATTRACTORS 2}

The 3D attractor's two sequence implements the same principles addressed in $3 \mathrm{D}$ attractor's one but on a more effective 3D platform. The grid of centre points is no longer limited to $\mathrm{X} \& \mathrm{Y}$ axis as further centre points are distributed along the $\mathrm{Z}$ axis, creating an even cubed grid of points ( $\mathrm{I}$ $\&$ II). This adds visual complexity and addresses further functional aspects of weight distribution. Iterations III through VII exhibit variations of weight distribution driven by various attractor points along the $X, Y \& Z$ axes. Finally, iterations VIII \& IX demonstrate the same function using alternative geometries. 


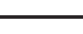

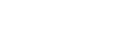




\section{SERIES FIVE}

SURFACE BOX MAPPING 
Fig. 37 $3 D$ attractors sequence two
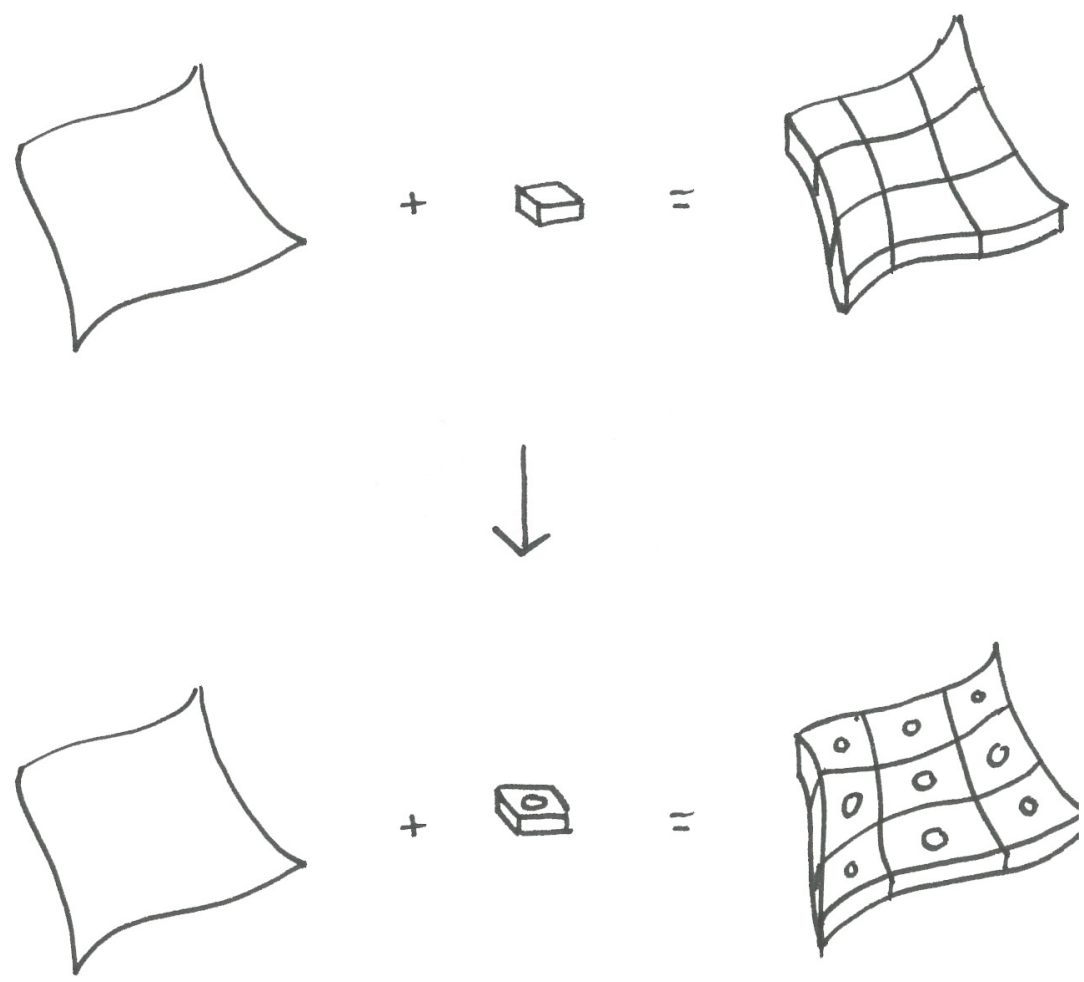


\section{SURFACE BOX MAPPING}

The surface box mapping series explores alternative methods for applying geometry to surface and object divisions. In a similar manner to the attractor surface experiments in series two, the free form surface is divided into a number of sections based on UV coordinates. However, instead of applying shapes to the divisions, a separate box geometry is mapped to the divisions (fig 37). This is effective for complicated forms as the mapped box morphs to the surface contours. If the external box is then modified, the corresponding mapped boxes are adjusted to inherit the same data. 


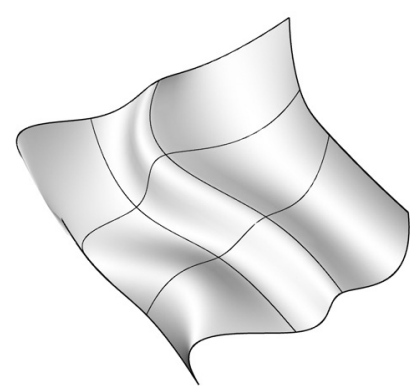

I

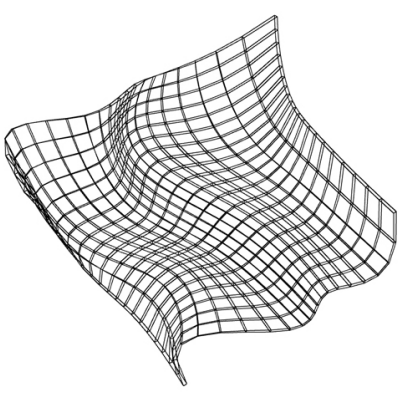

IV

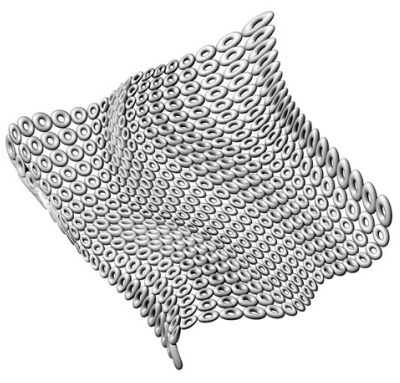

VII

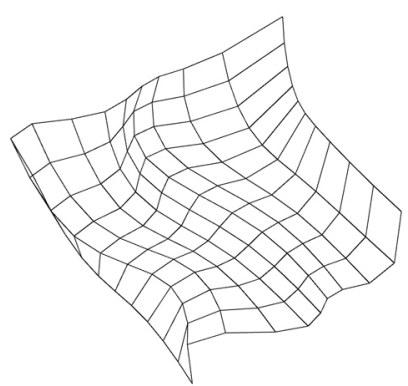

II

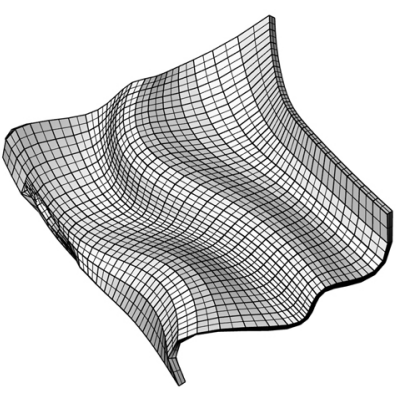

V

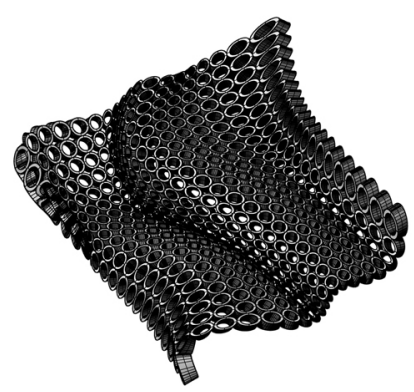

VIII

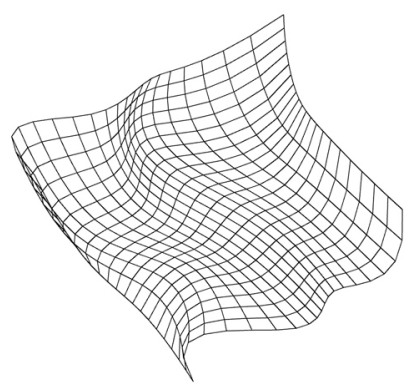

III

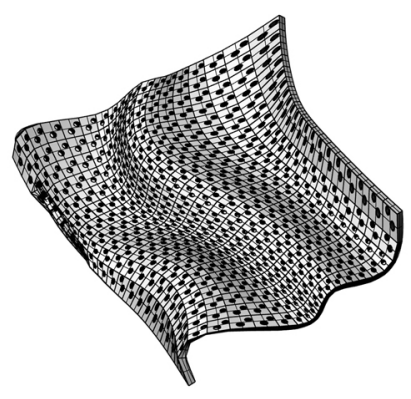

VI

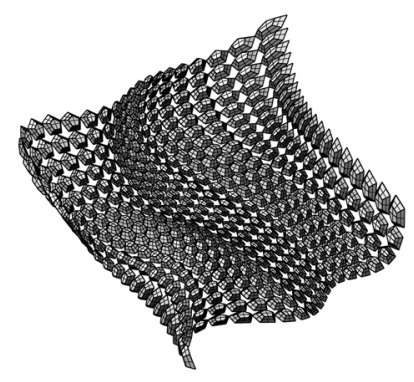

IX 


\section{SURFACE BOX MAP 1}

The surface box map one (fig 38) sequence explores how alternative box geometries respond to being mapped across a surface and the feasibility of these objects to be manufactured. It should be noted that if a free form surface is divided unevenly between $\mathrm{U} \& \mathrm{~V}$ coordinates, the division will take the form of a rectangle rather than a square, as seen in iterations I, II \& III. Iteration IV exhibits the a line drawing of where the box is intended to fill while $\mathrm{V}$ shows the solid object. VI through IX explore variations of this box, from a simple hole (VI) to a doughnut form. It is noted that any form that doesn't connect to at least four flat sides is unable to be manufactured. 


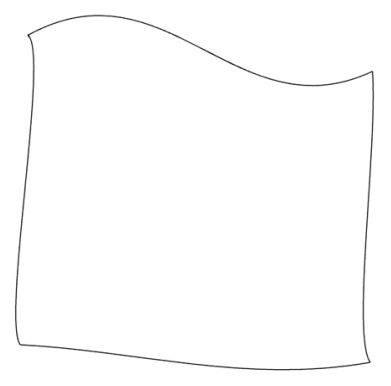

I

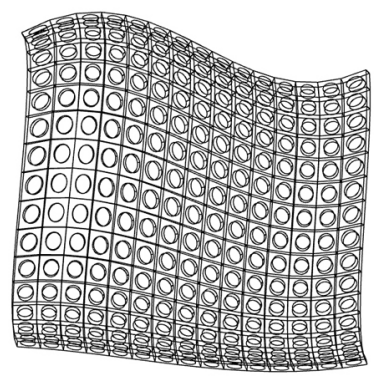

IV

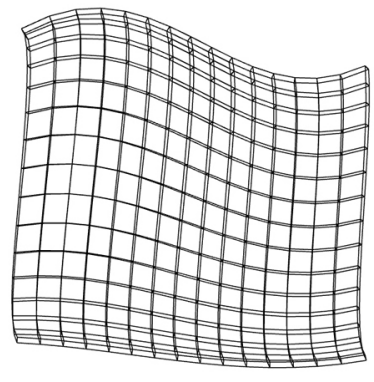

II

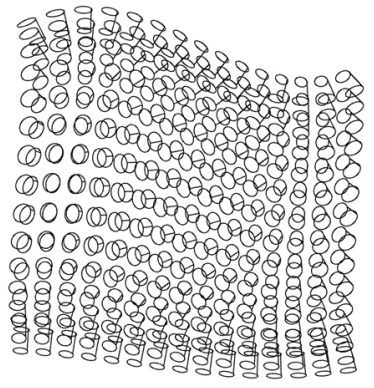

III

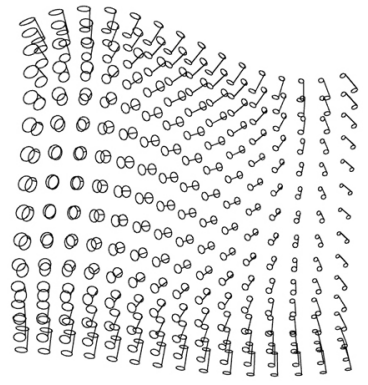

V

VIII

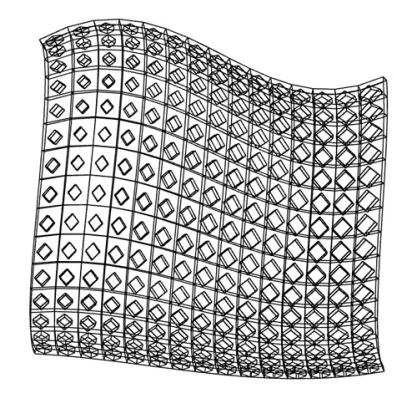

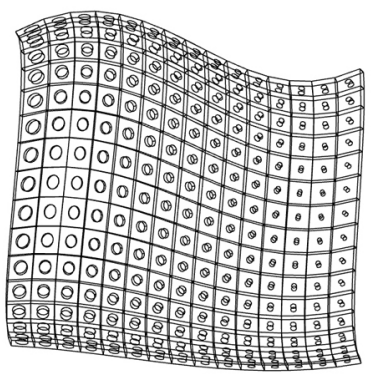

VI

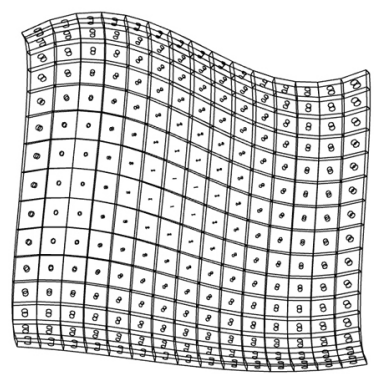

VII 


\section{SURFACE BOX MAP 2}

The surface box map two (fig 39) explores the basic box mapping technique explored in surface box map one, however integrates the attractor point system for distributing mass. Using attractor's with box mapping is quote different as there is no point grid, and item to adjust is a separate entity. The free form surface (I) is divided by UV mapping coordinates (II), but instead of applying a standard box, the geometry to be adjusted is used (III). The box is then applied with the cylinder subtracted from the original form (IV). A relationship can then be placed between the size of this cylinder and the distance between attractor point and divided surface. Iterations V through VIII display the results of adjusting the attractor point location and associated restraints. IX shows the form and a manufacturable mesh. 


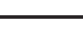

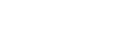


SERIES SIX

3D MESH FORM

$-123-$ 
Fig. 40| 3D mesh sketch

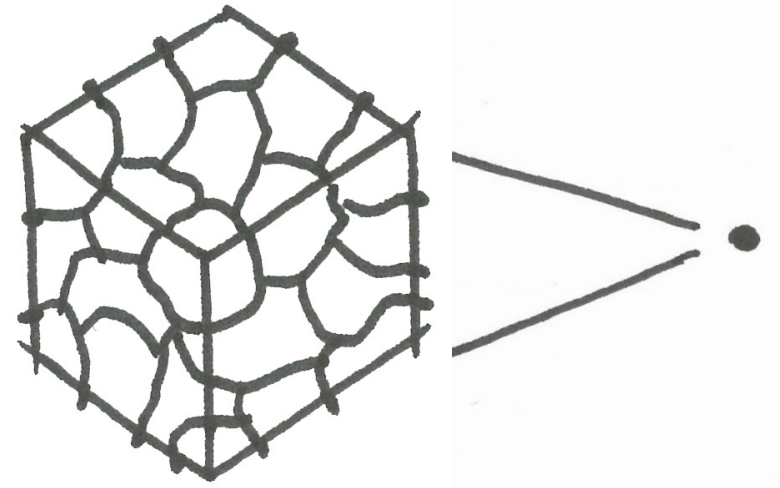




\section{D MESH FORM}

The mesh-form series explores the application of mesh tools on the outer surface of a $3 \mathrm{D}$ object. These objects are divided into a series of curves which can be further transformed to produce mesh objects. Mesh-form one explores piping tools to create a cylindrical structure from a series of curves while mesh-form two takes a divergent approach exploring a range of tools from all previous experiments. This series provides the first experimental insight into true $3 \mathrm{D}$ objects. 

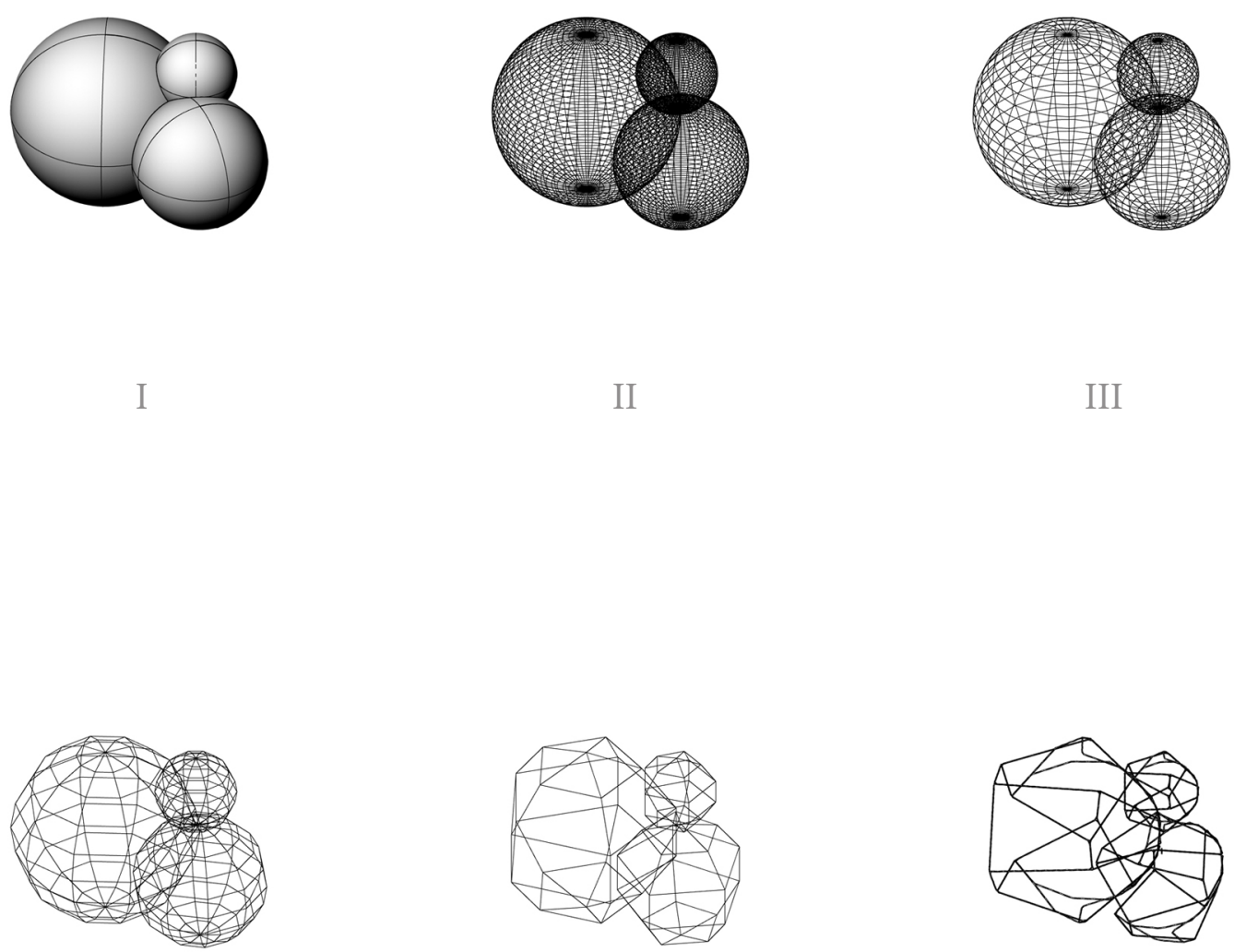

IV
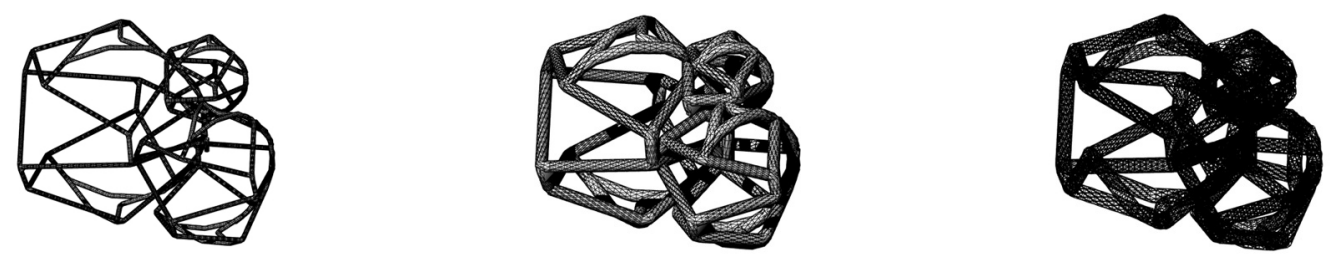


\section{MESH FORM 1}

The mesh-form one (fig 41) sequence explores breaking down an object to its absolute essentials. Inspired by the geometric category of the digital form guide, each iteration reduces a surface into a 'low-res' series of curves (iterations I through $\mathrm{V}$ ). Wire-frame thickening and 'topologizer' tools have been implemented to discard any excess curves and give them a solid thickness (iterations VI through IX). It is noted that this would be an effective tool for optimizing functional geometry. 

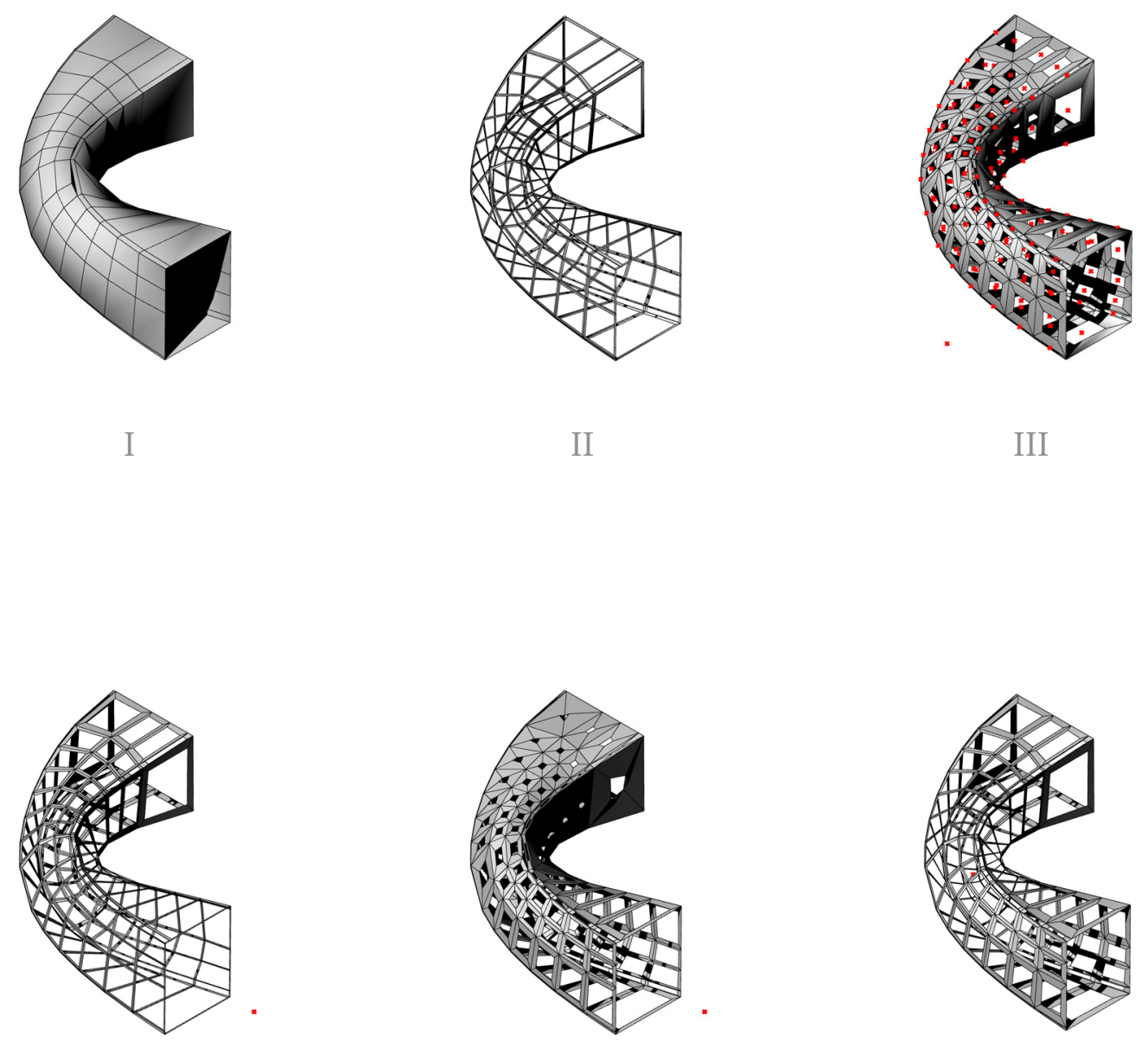

IV
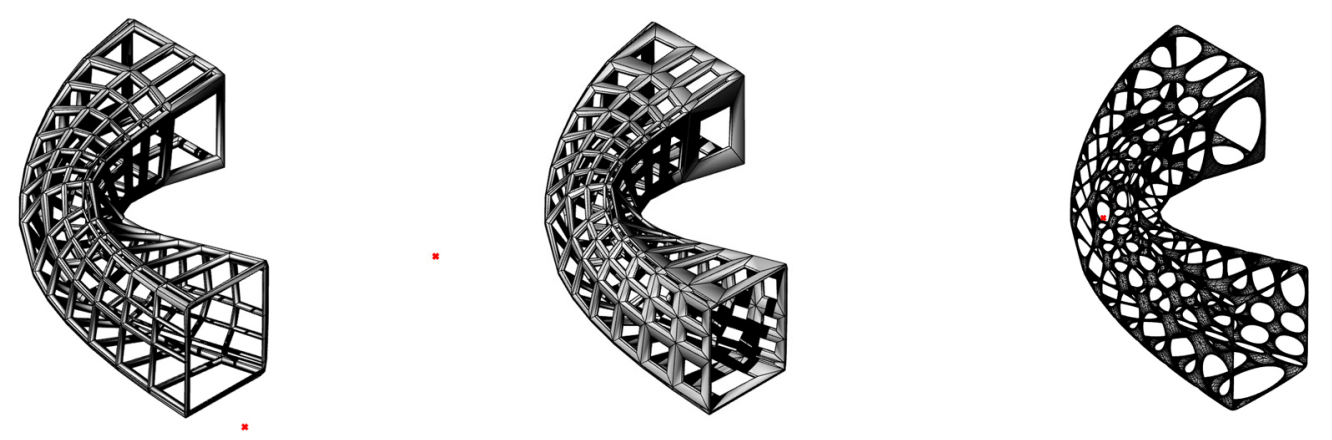


\section{SURFACE MESH FORM 2}

The mesh-form two (fig 42) sequence combines a range of techniques employed throughout multiple previous experiments. The $3 \mathrm{D}$ object is divided by UV coordinates (I) and minimized into a wire-frame model (II). The wireframe model then uses lofting techniques explored previously between scaled geometry and surface division curves. Attraction points are added in conjunction with appropriate constraints to test theoretical weight distribution (III, IV, $\mathrm{V} \& \mathrm{VI})$. Geometries are then transformed into a mesh and given a thickness for manufacturing purposes (VII \& VIII). Finally iteration IX employs an organic pattern as mentioned in another experiment. 
Fig. 43| Mesh form sequence three
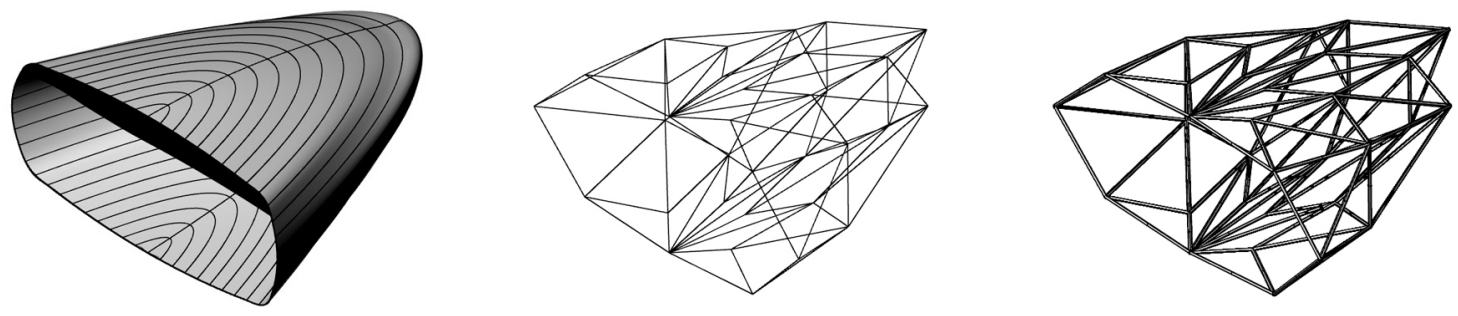

II

III
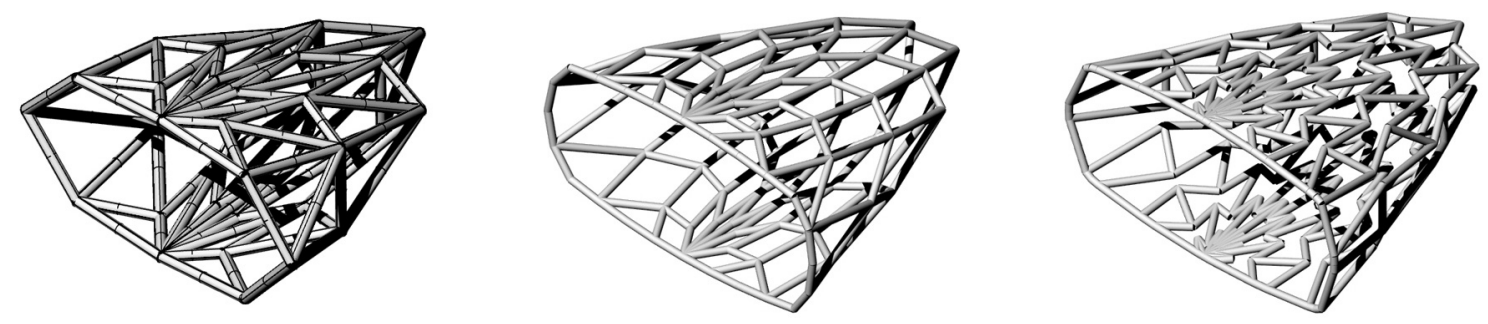

IV
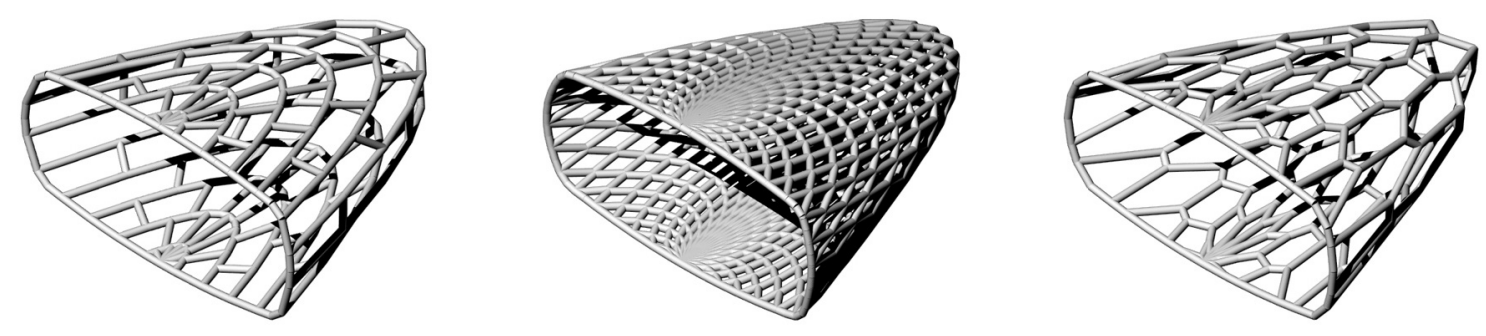

VII 


\section{SURFACE MESH FORM 3}

The mesh-form three (fig 43) sequence combines the same techniques as mesh form three. However, in this case it is applied to a geometry that could begin to resemble a golf club/putter. It was determined from this experiment that although it is a visually interesting form, it would not work very well for the distribution of weight and other functional criteria.

Fig. 44| Visual composition of experiments

(see next page) 

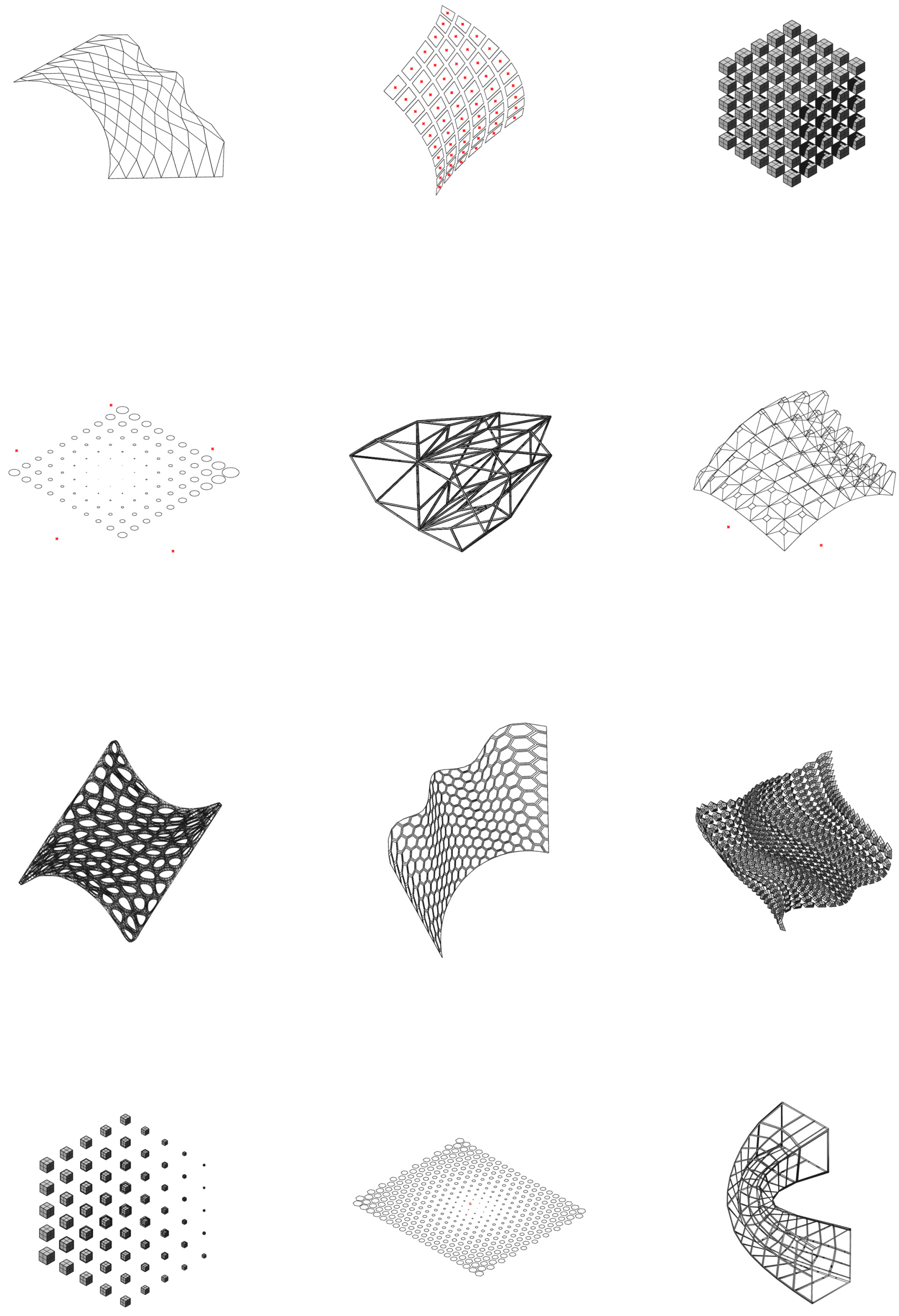

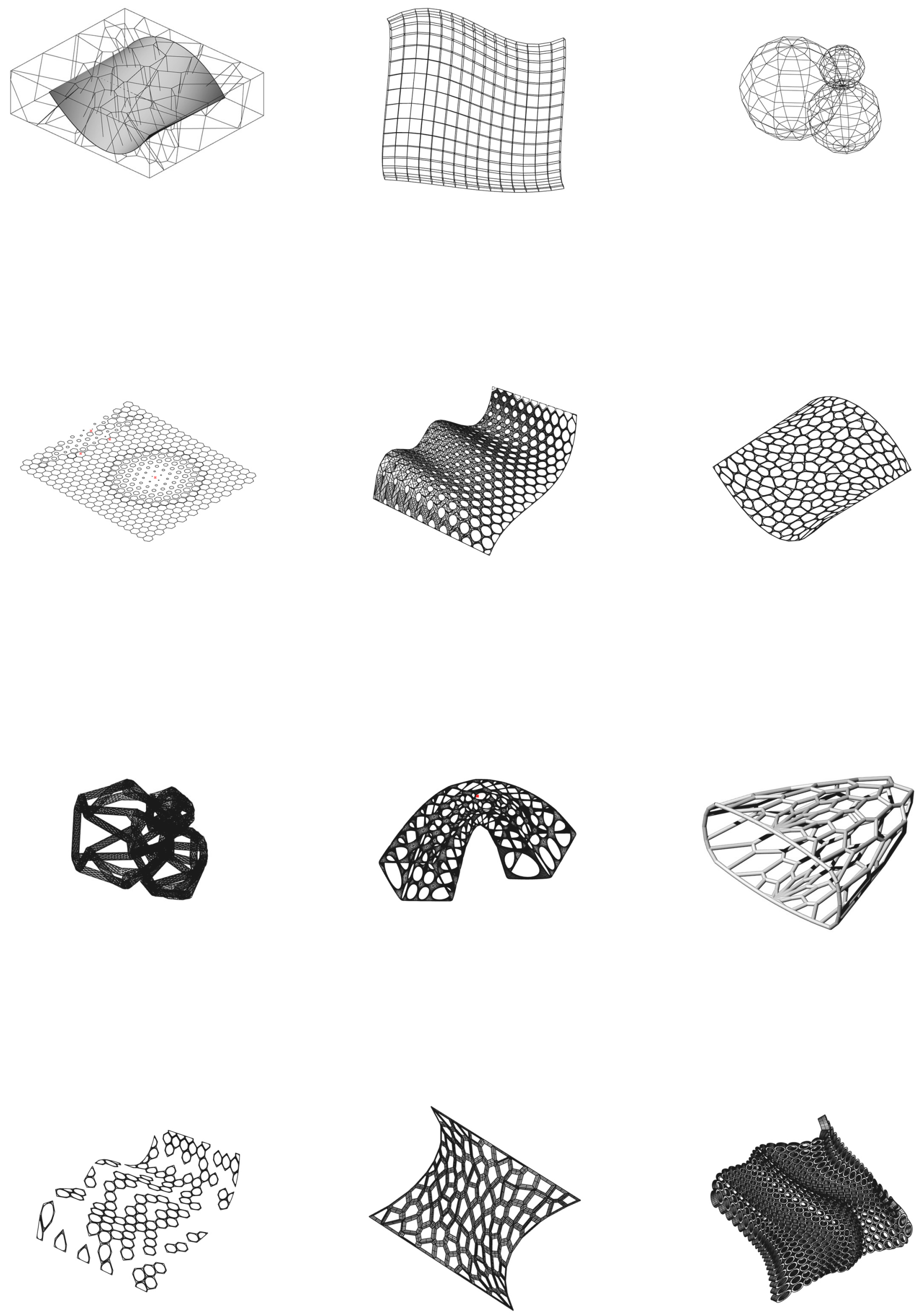


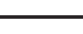

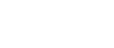




\section{CHAPTER SIX}

APPLIED EXPERIMENTS 
Fig. 45 | Applied experiment sketches

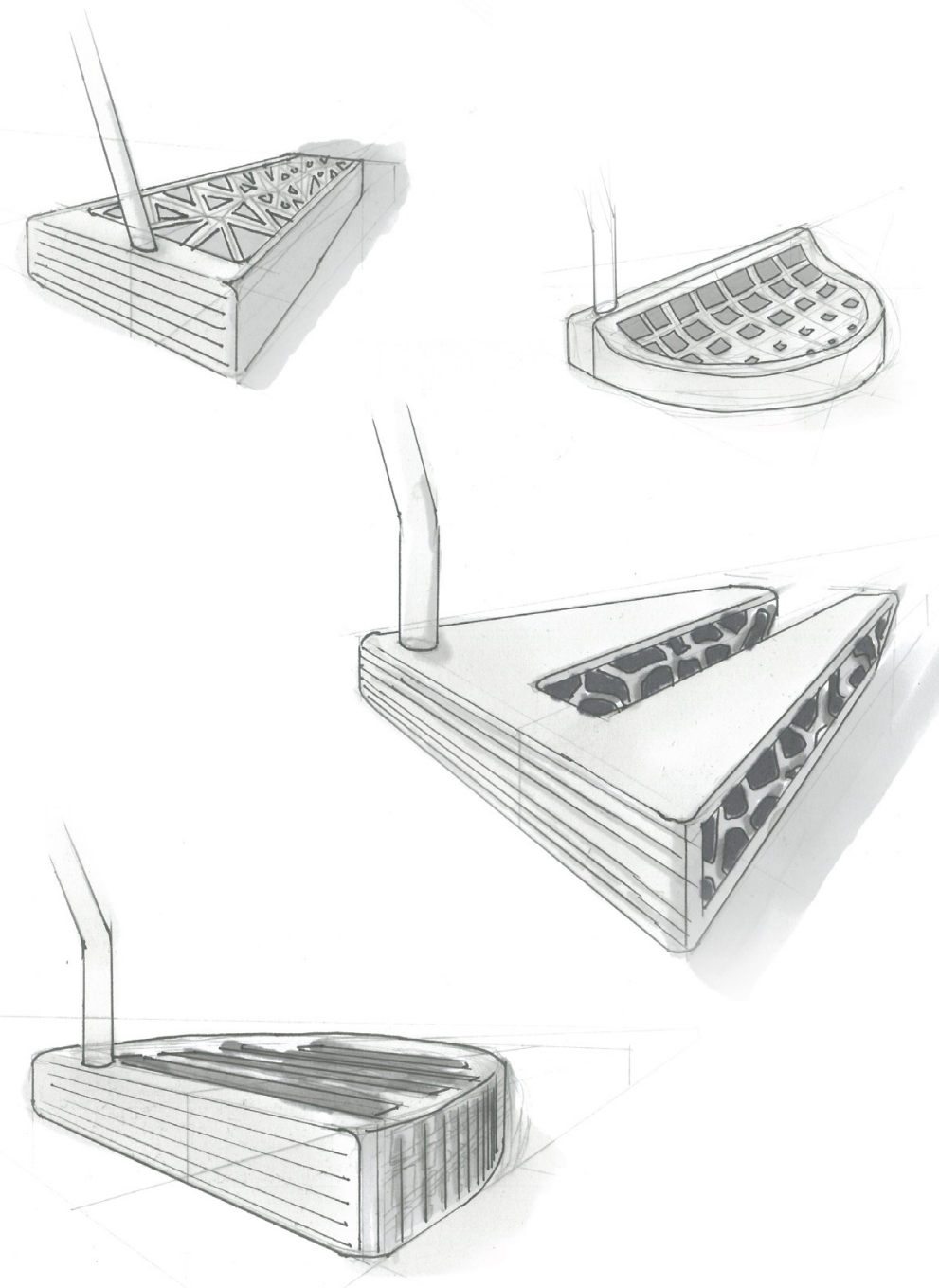




\section{APPLIED EXPERIMENTS}

The applied experiments chapter takes functional issues surrounding golf club design and test these criteria using digital tools described in the programme analysis. These discrepancies include loft, lie, weight distribution and dimensional restraints put in place by the United States Golf Association (USGA). These issues will be confronted by applying productive parametric modelling techniques used throughout the initial experiments stage. 


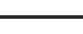

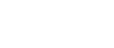




\section{USGA PUTTER RULES}

The United States Golf Association has many rules in place for every club in the bag. Their main objective "is to ensure that technological advances in the design and manufacture of golf equipment are in the best interests of the game" (USGA, 2015). This research will abide by these rules and use them as guidelines for designing parametric models.

The club head's width, measured from the outermost portions of the putter's heel and the toe, cannot exceed 7 inches $(177.8 \mathrm{~mm})$.

The club head's height, measured from the sole to the uppermost portion of the club head, may not exceed 2.5 $(63.5 \mathrm{~mm})$ inches.

The club head's width must be greater than its depth, measured from the face to the back of the club head.

The club face's width must also be greater than or equal to two-thirds of the club head's depth.

A putter is defined as "a club with a loft not exceeding ten degrees designed primarily for use on the putting green.

As with all clubs, the angle of a putter's shaft relative to the ground cannot exceed 80 degrees when the club is in the address position. 
Fig. 46| Comparable loft \& lie
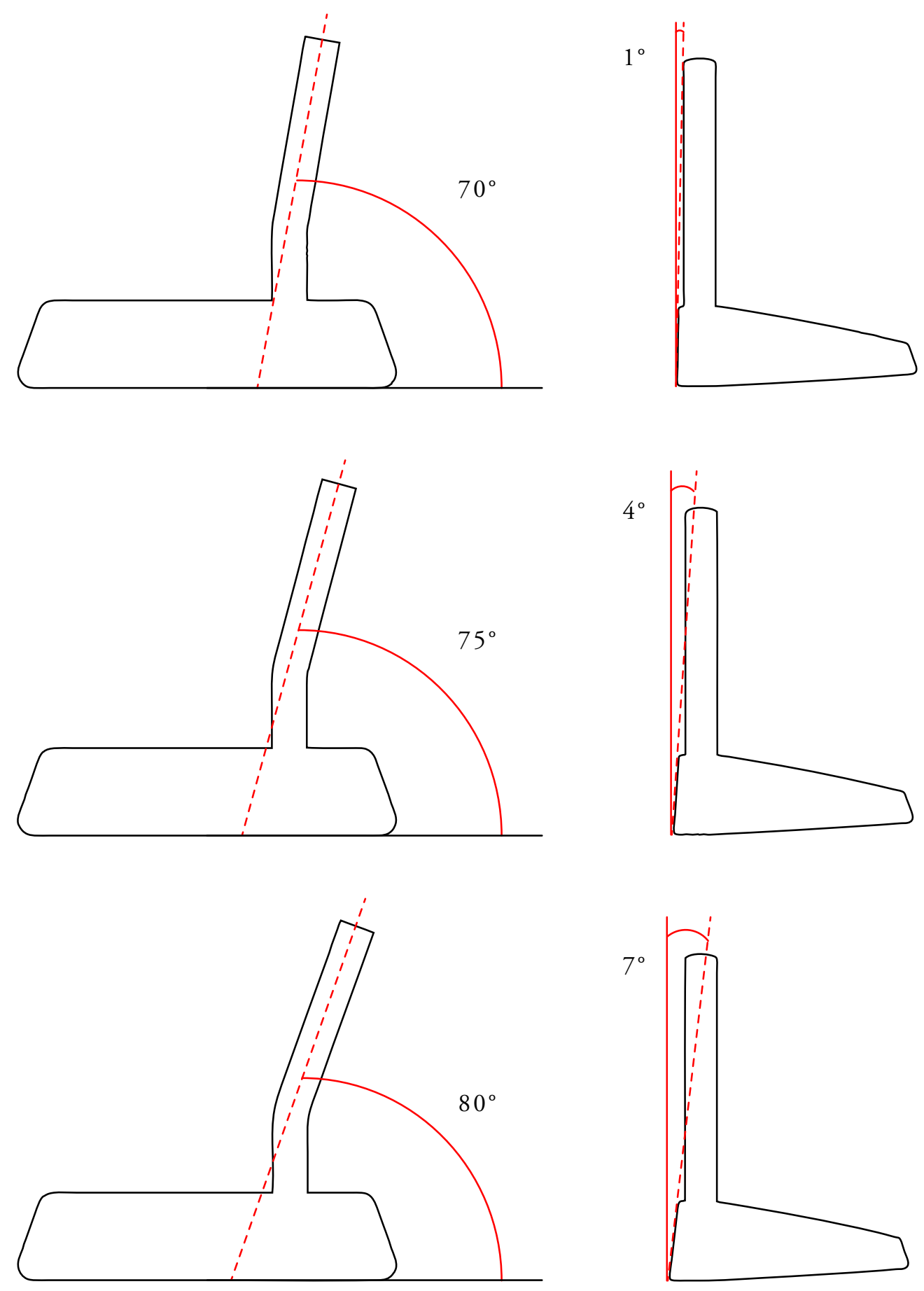


\section{PARAMETRIC LOFT \& LIE}

Lie angle and loft angle are both limited to a single axis of rotation where the sum can either increase or decrease. Therefore, a simple angle parameter controlled by a number slider can cater for any individual The only restraints being the minimum and maximum angles as conforming with the USGA rules.

Lie angle is entirely related to directional control. Variability in lie angle can determine whether a shot is pushed or pulled by changing the clubface plane.

Face loft angle is entirely related to distance control. Variability in loft angle determines the skid to roll percentage of your putt and can control whether you consistently hit the ball short or long.

Fig 46 shows a comparative image of both of these variations that would suit different user requirements. 


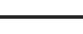

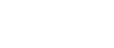




\section{CLUB FORMS}

A number of basic putter head forms were trialled with specific constraints, relationships, data inputs and computational aesthetics.

In the first example, the club takes its form as an abstracted geometry of putters in the present market. Parameters such as the USGA rules, loft and lie were applied to all experiments, but the theoretical data was independent with each club. Each iteration considered how digital aesthetics discovered in the preliminary experiments could aid the geometry in distributing weight appropriately.

The following example shows a range experimental of aesthetics applied as functional inserts to work with the parametric clubs mentioned above. Each experiment combines individual data with the computational design aesthetics explored in the preliminary experiments.

Finally, examples of both club forms and aesthetic inserts are combined in an experimental range, over a number of iterations. 
Fig. 47 | Parametric iteration options (functional criteria)

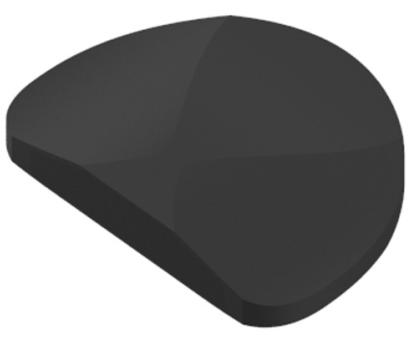

I

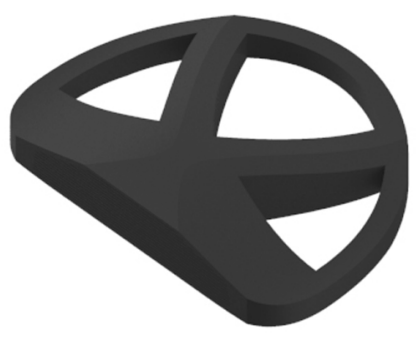

IV

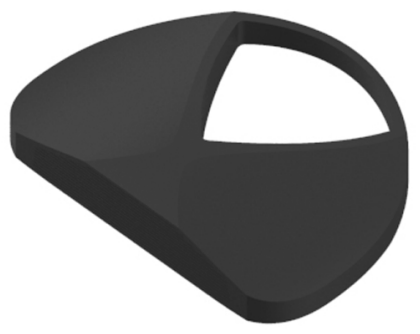

VII

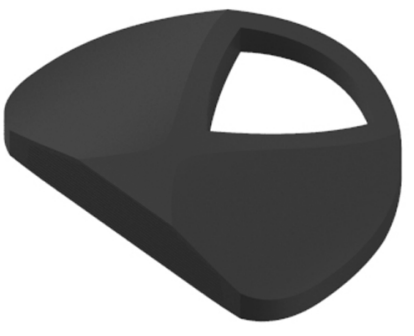

II

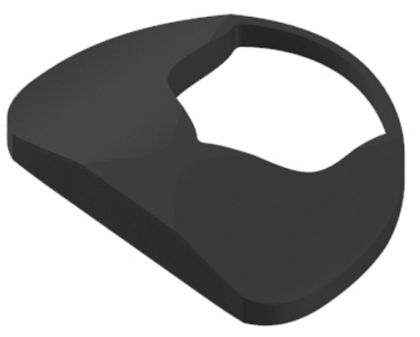

V

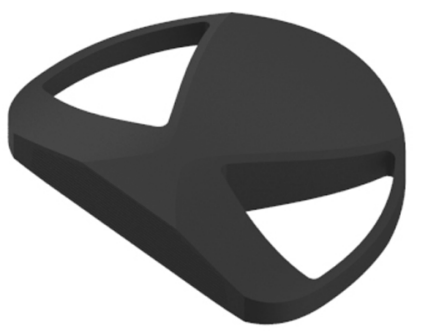

VIII

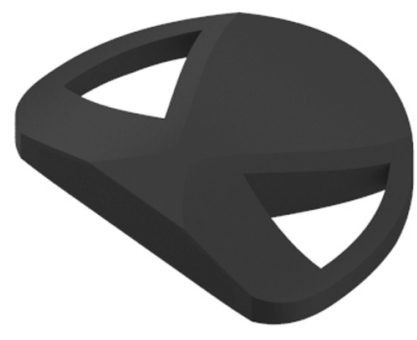

III

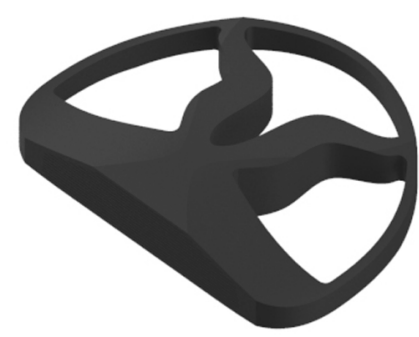

VI

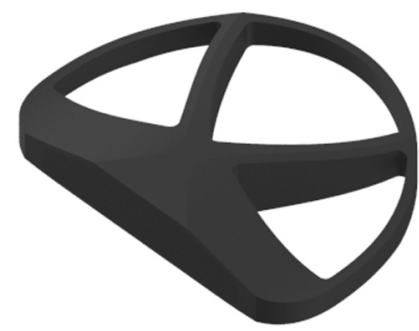

IX 
The sequence of iterations in Fig. 47 explore the potential for weight distribution around the clubhead of a typical malletstyle putter (I). Each iteration represents a different player and a different stroke style, based on their data. For example, iteration III would be suited for a player who requires a centre weighted club, with a central COG whereas iteration $\mathrm{V}$ would suit a player who requires a perimeter weighted club with a forward COG. Each adjustment shown in this sequence is the result of existing parameters and constraints set in a parametric model. At this stage the clubs are determined $50 \%$ by function (physical performance aspects) and $50 \%$ aesthetic preference (mental performance aspects). The next step is to apply the parametric inserts that meet both functional and aesthetic criteria for customisation. 


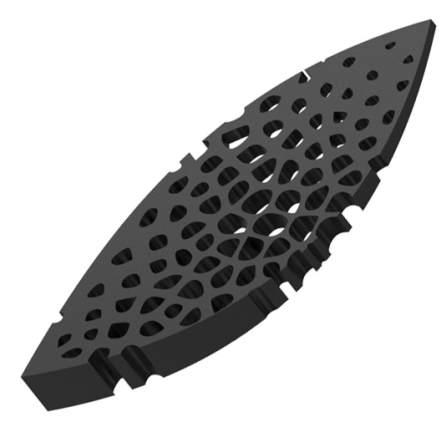

I

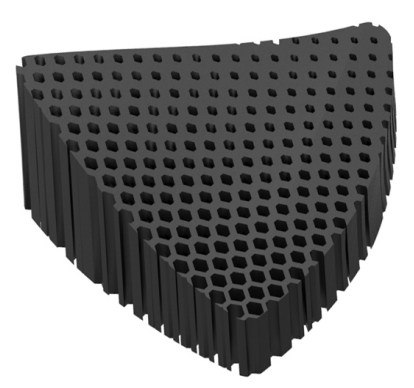

IV

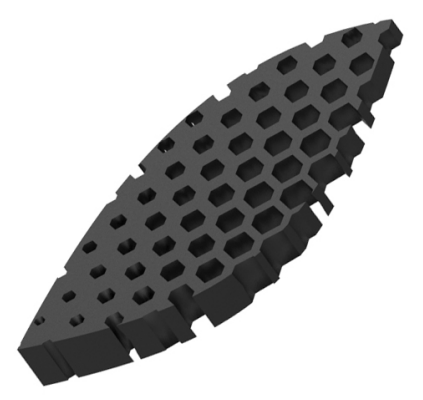

VII

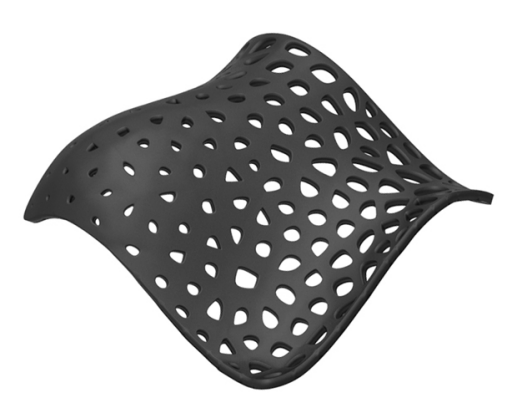

II

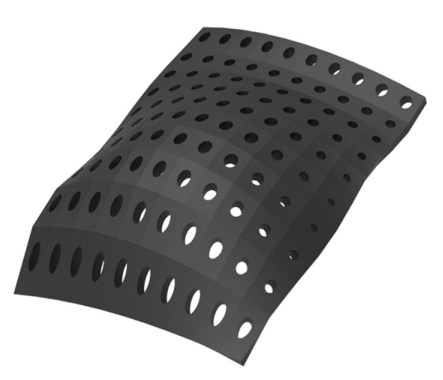

$\mathrm{V}$

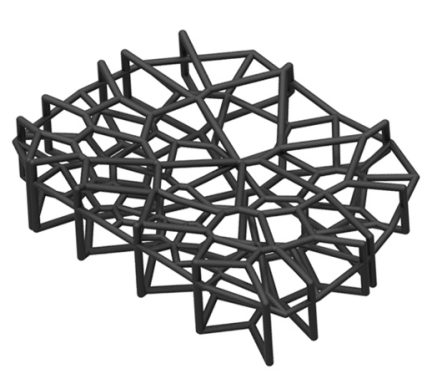

VIII

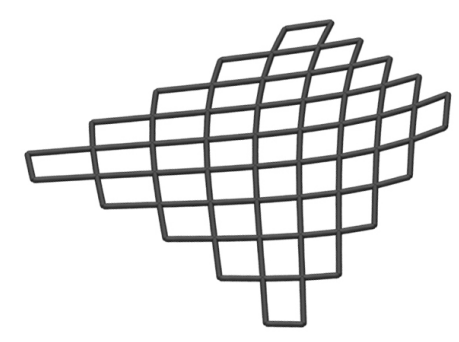

III

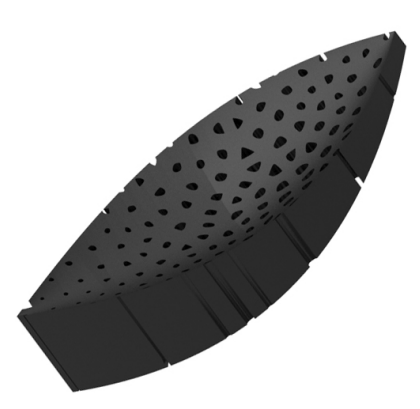

VI

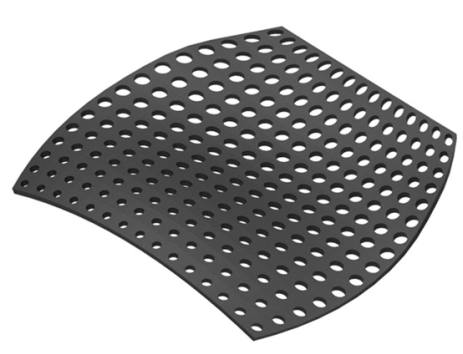

IX 
The sequence of iterations in Fig. 48 explore the computational design aesthetic potential to meet functional criteria of an individual's data. The examples shown are only a handful of the iterations made. Each one is an example of combining computational design aesthetics (preliminary experiments) with $3 \mathrm{D}$ geometry for inserts, such as seen in Fig. 47 and driven by individual user data. The next step is to combine these inserts with some of the parametric club forms. 

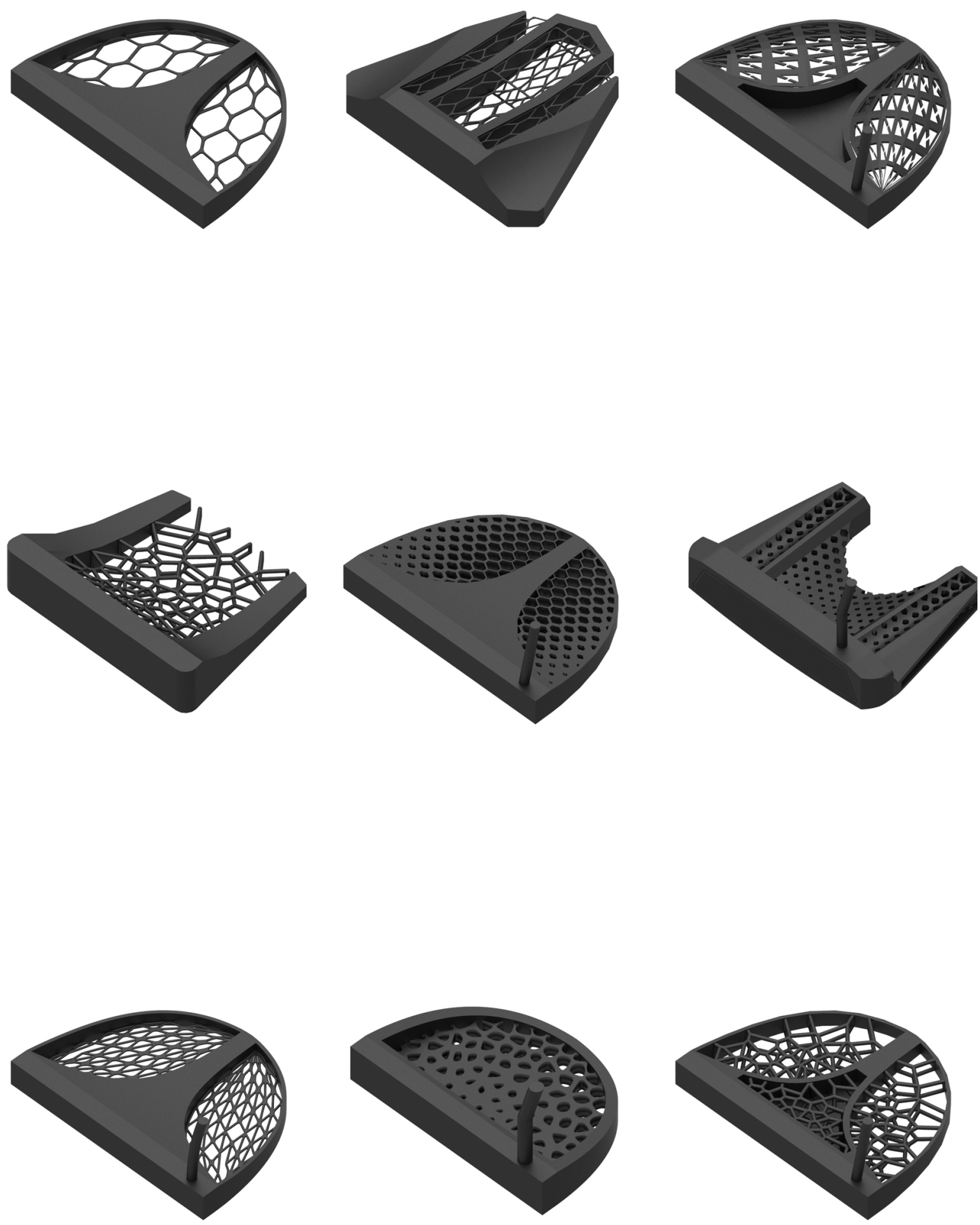
This final step is the most important when it comes to customisation. It combines the general data related geometry with the more refined data based insert. Resulting in an output unique to the individual's mental and physical performance criteria.

Fig. 49 exhibits a range of various putter head concept designs. Each putter explores a unique combination of computational design aesthetic and weight distribution. These examples are only a handful of development concepts, but each was just as helpful as the next to move into the next stage.

Each concept, like the preliminary experiments provided valuable information on the effectiveness and quality of digital tools. These were then able to be taken into the next experiment and so forth.

The next chapter presents preliminary designs that draw on the collective knowledge of all experiments to date. 


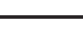

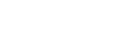




\title{
CHAPTER SEVEN
}

\author{
PRELIMINARY DESIGNS
}




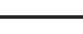

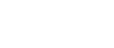




\section{PRELIMINARY DESIGNS}

Five applied experiments were selected based on their differentiated club form (determined by a different set of data) and choice of computational design aesthetic. Each preliminary designs explores the advantages and disadvantages of applying a specific aesthetic to drive functional performance.

The selected designs have been intentionally constructed to be manufactured as a 1:1 scale, tangible object. These models were fabricated at Shapeways (the world's leading 3D printing service) in a white, strong \& flexible nylon plastic. White was specifically chosen so the form could be examined without any other visual distractions.

Each of the five preliminary designs have a face loft parameter of $3^{\circ}$ and a lie angle of $71^{\circ}$ to match the swing data of the designer, therefore controlling consistency across the range. 
Fig. 50| P1 function + aesthetics

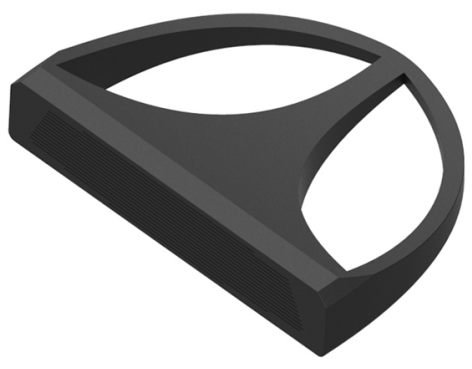

$+$

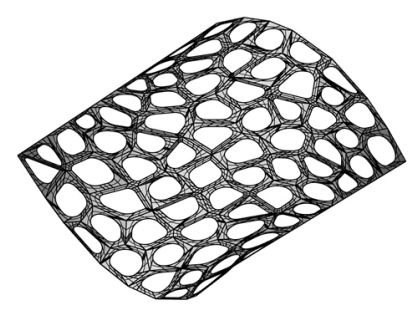

$=$

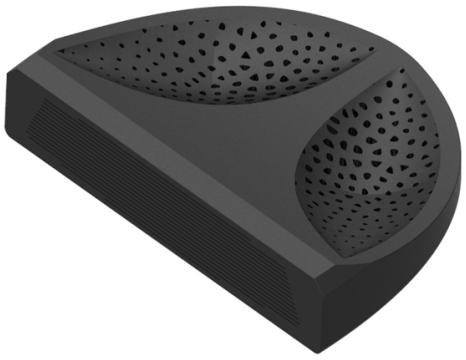




\section{PRELIMINARY ONE}

Preliminary design one explores the functional potential of organic voronoi aesthetic. Drawing on findings from series three (voronoi meshes) and series one (attractor points), this experiment aims to effectively utilize the mathematical power of voronoi diagrams.

This experiment employed two fundamental parameters: the opening size of the voronoi geometry and the overall mass of the voronoi geometry which were determined by how much weight was needed in a particular area and would work simultaneously to produce the geometry (both defined by attractor points).

It was found that the combination of parameters worked very well together in terms of general weight distribution addressing individual data requirements. However the application of voronoi aesthetic caused some problems leading up to the design. Due to the nature of voronoi modelling tools, the automation of mathematical function made it hard to be extremely accurate in terms of exact weight required.

When an attractor point was moved, the voronoi cells would find the best new fit around those points. This proved inconsistent as even the slightest of changes to the coordinates of a point could give two, quite different geometries. 
Fig. 50| P1-3D printed model
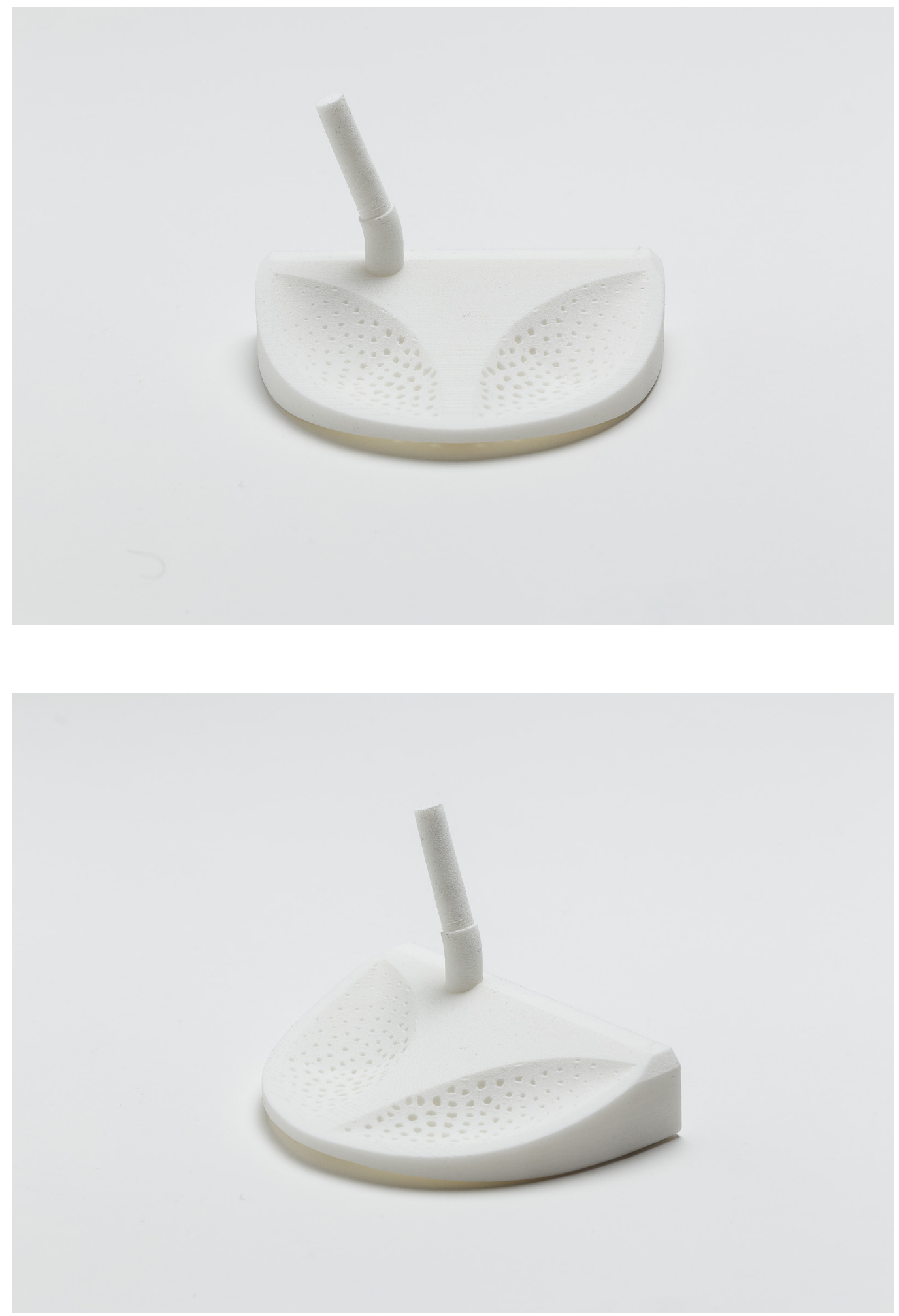
Fig. 51| P1 object and detail shots

$-157-$ 
Fig. 52| P2 function + aesthetics

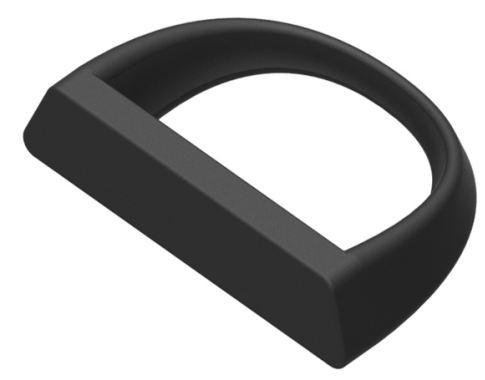

$+$

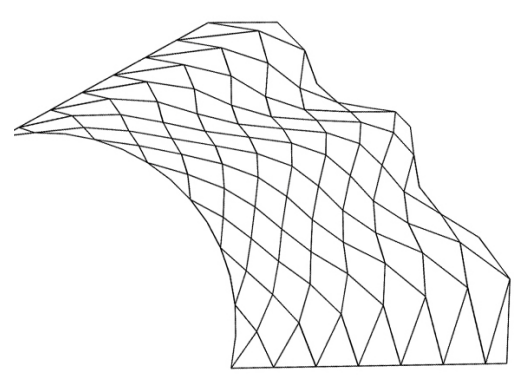

$=$

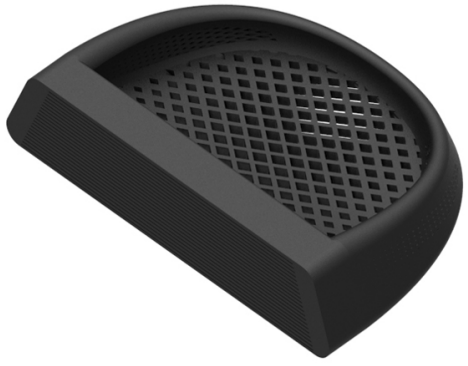




\section{PRELIMINARY TWO}

Preliminary design two explores functional potential of diamond panelling tools across a geometries surface. Utilizing knowledge and implementing parametric scripts tested in series two (attractor surfaces), this experiment aims to visually communicate intended function.

This experiment applied two major parameters: the scaling of diamond aesthetic would increase where weight needed to be reduced and a reflection of this was applied to the framework of the club. The intent behind this was to produce a geometry whose function could be read from angle.

This particular club inherited data from what would be an inconsistent player. As described in the site analysis, a player who strikes the ball all over the face requires a high moment of inertia or perimeter-weighted club. It was found that the first parameter was successful in creating a visual language to express the particular function defined by the individual data.

However, it was also noted that the second parameter was not so successful. Although it made for a aesthetically pleasurable detail, it lost its functional purpose. This was due to the scale of said geometry as such an intricate form could not be effectively fabricated. 
Fig. 53| P2-3D printed model
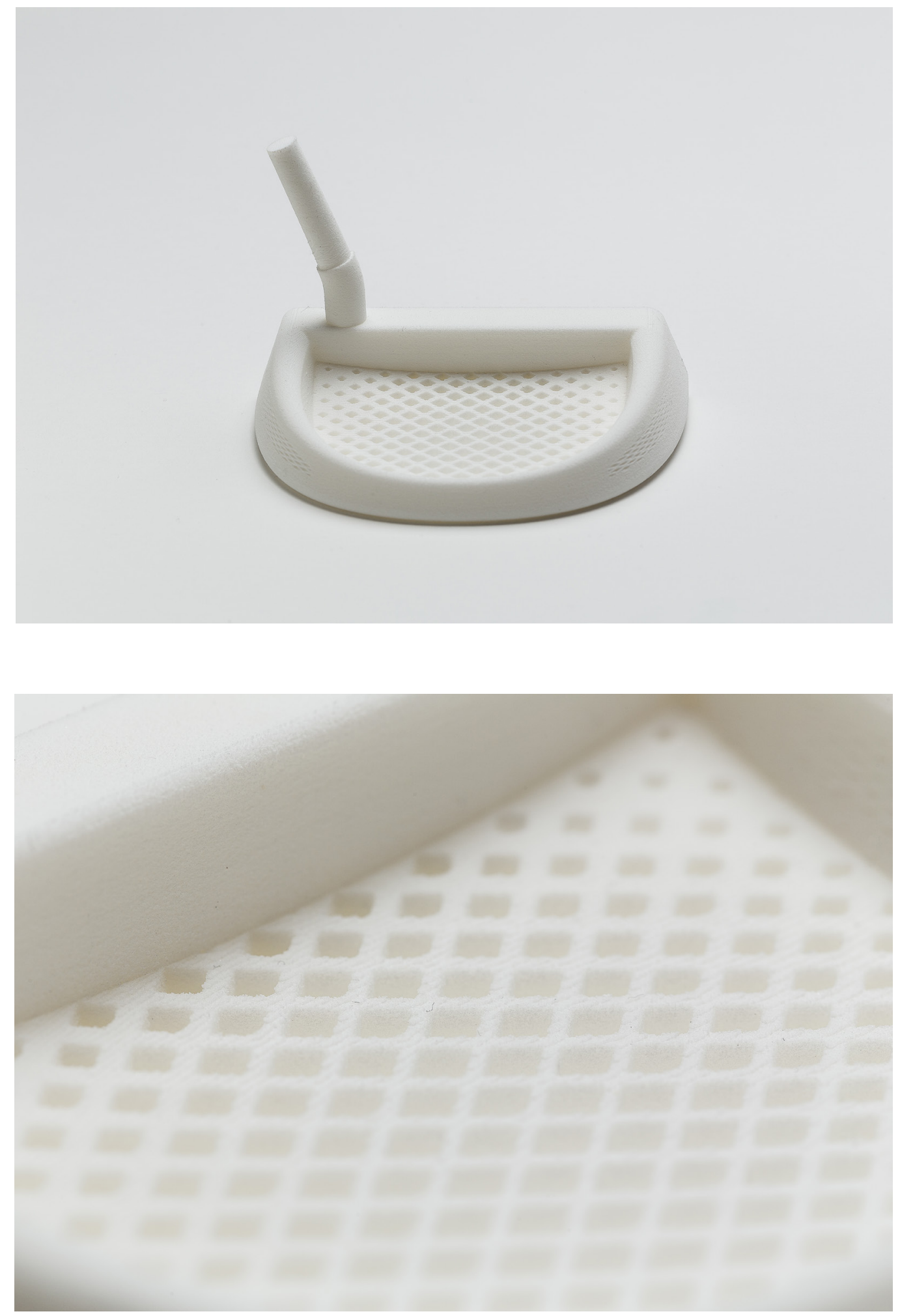
Fig. 54| P2 object and detail shots
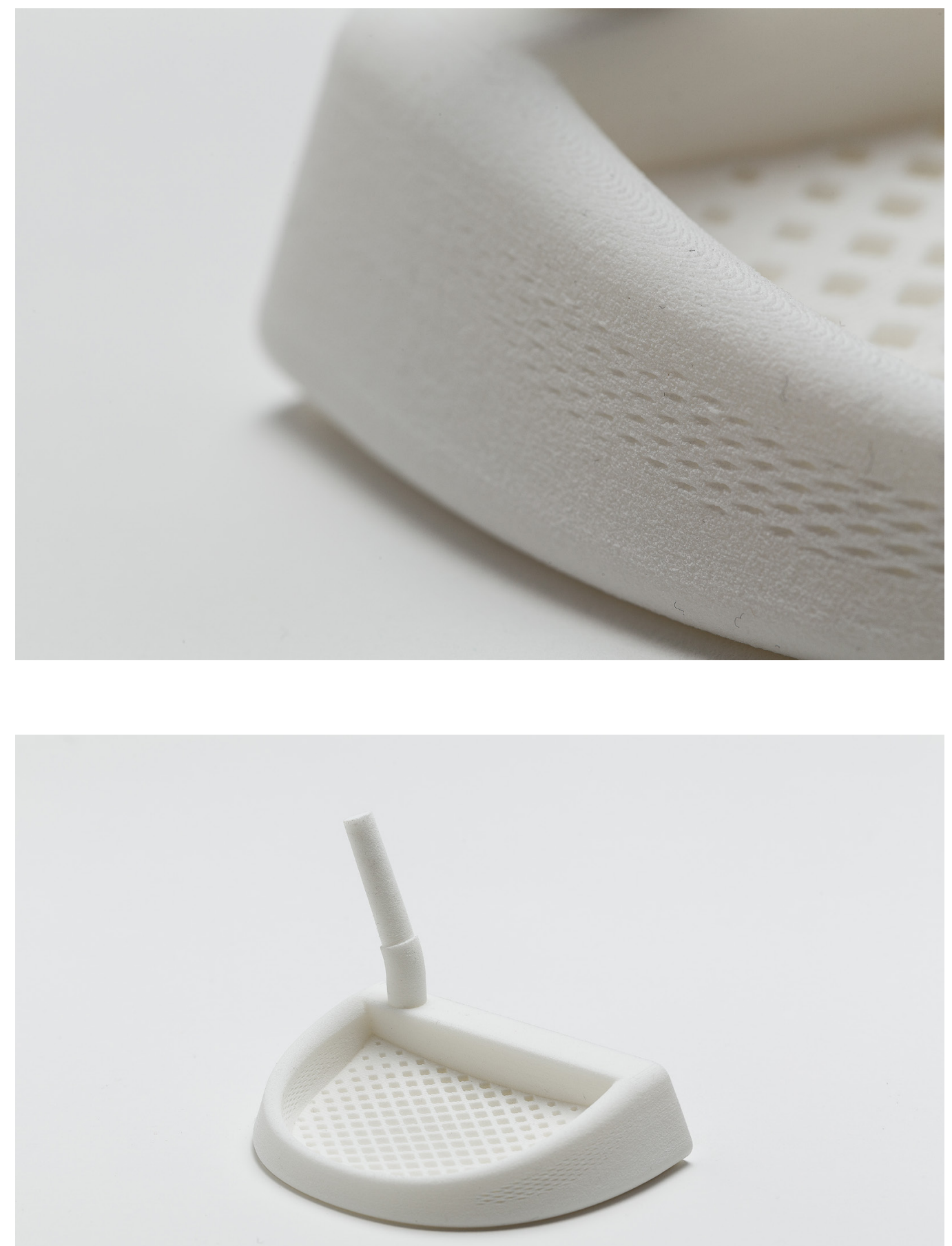


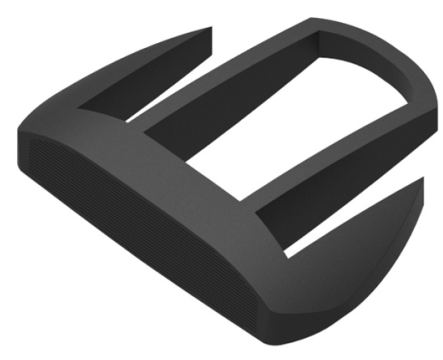

$+$
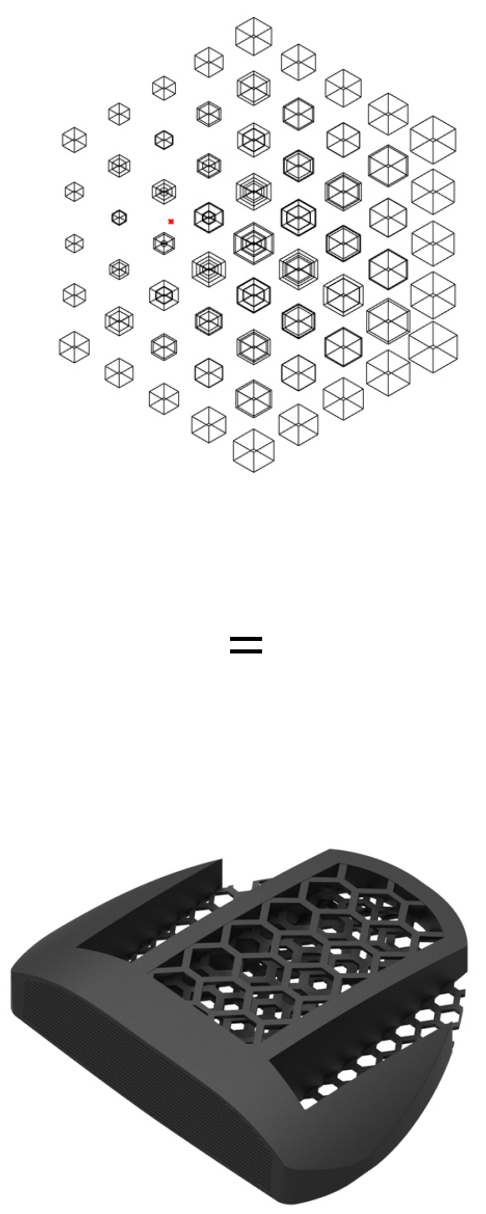


\section{PRELIMINARY THREE}

Preliminary design three explores the functional potential of layering geometry based on gradual density. Serendipitously inspired by the visual language of iteration I in 3D attractor cubes 2, this experiment aims to distribute weight through multiple surfaces, to create an extra 3D complexity.

This experiment trialled a parametric script which combined algorithms used in mesh form 2 and attractor surface 1 experiments. The main parameter employed adjusted the scaling of each layer of within the central geometry based on the required positioning of the centre of gravity. This parameter was determined by attractor points moving up and down the $Z$ axis. In this particular model, the centre of gravity was positioned low and central to the geometry.

It was found that the layering technique worked well in creating both an extra 3D complexity as well as an aesthetic exclusive to computational design. The layering technique also worked well in effectively positioning the centre of gravity, however it was limited in distributing weight functionally around the rest of the clubhead. 
Fig. 56| P3-3D printed model
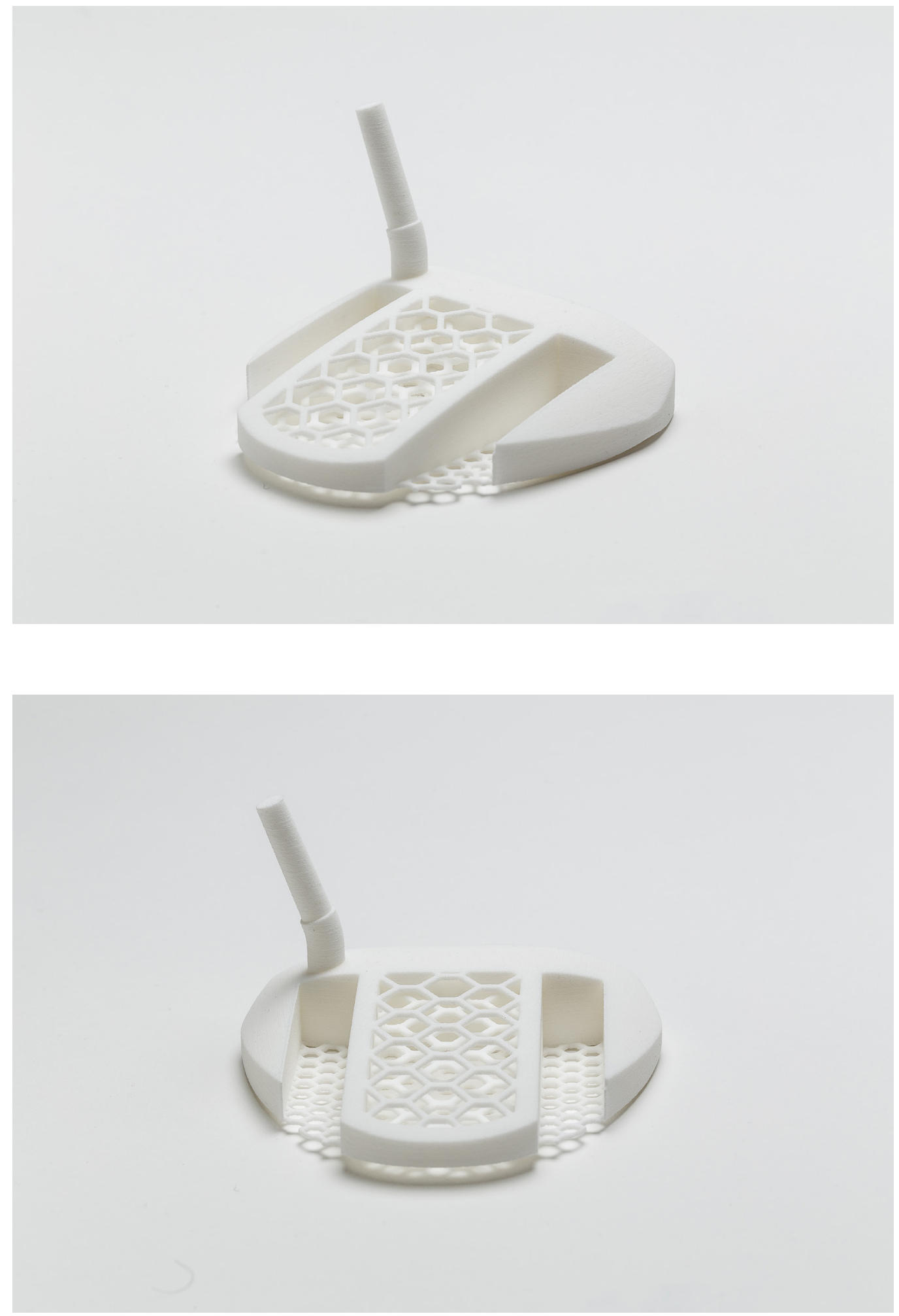
Fig. 57| P3 object and detail shots

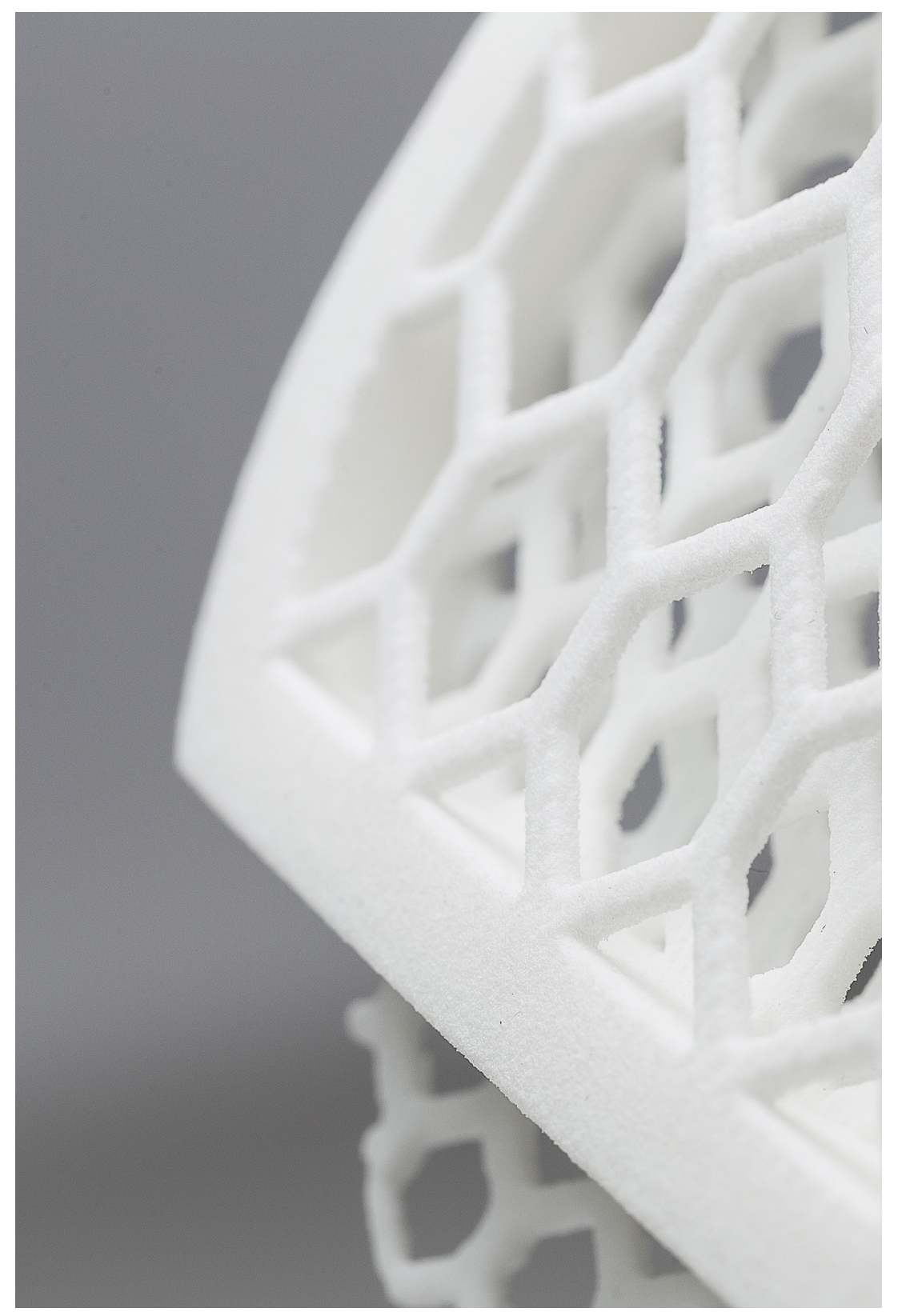

$-165-$ 
Fig. 58| P4 function + aesthetics

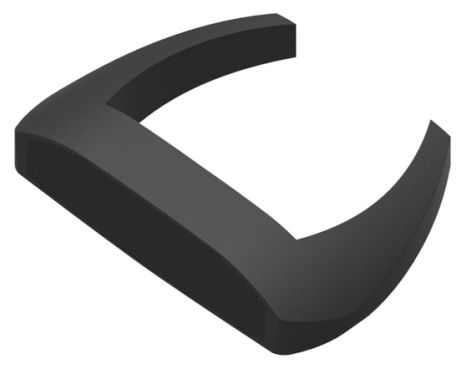

$+$

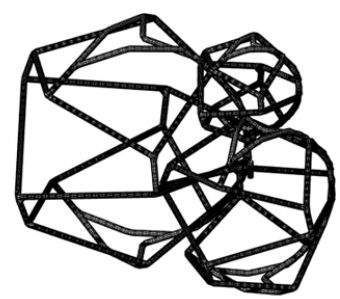

$=$

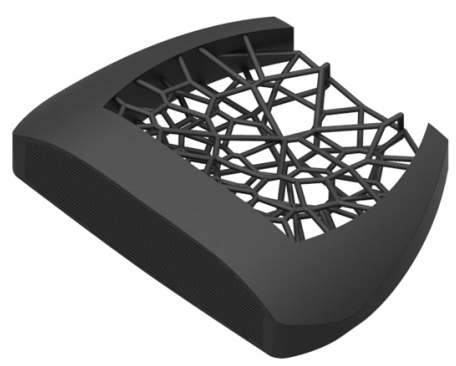




\section{PRELIMINARY FOUR}

Preliminary design four, like preliminary design one (pd1) explores the functional potential of the organic voronoi aesthetic. However, dissimilar to pd 1 this experiment also draws on findings from surface mesh-form sequences $1 \&$ 3. This concept aims to apply piping techniques to the parametric script to create further aesthetic and functional complexity.

This experiment employs two main parameters: the density each piped voronoi layer and the number of layers in total. Both parameters are related to the positioning of attractor points in relation to distribution of centre of gravity (COG) and moment of inertia (MOI). Attractor points were positioned along the $\mathrm{X}, \mathrm{Y}$ and $\mathrm{Z}$ axes to ensure both parameters would be effectively addressed. This particular example exhibits are medium front-middle COG and a medium MOI, relating to a slightly more consistent player than the previous two concepts.

It was found that the issues surrounding voronoi calculations in pdl were no longer a problem through the adapted parametric script. In fact, it was quite the opposite. The issue of inconsistency was eliminated through the multiple layering as each adjustments was averaged out by the next layer. However, it was found that the piping tool was only useful for tiny refinements in weight as the pipes were incredibly accurate but carried little weight. 
Fig. 59| P4-3D printed model
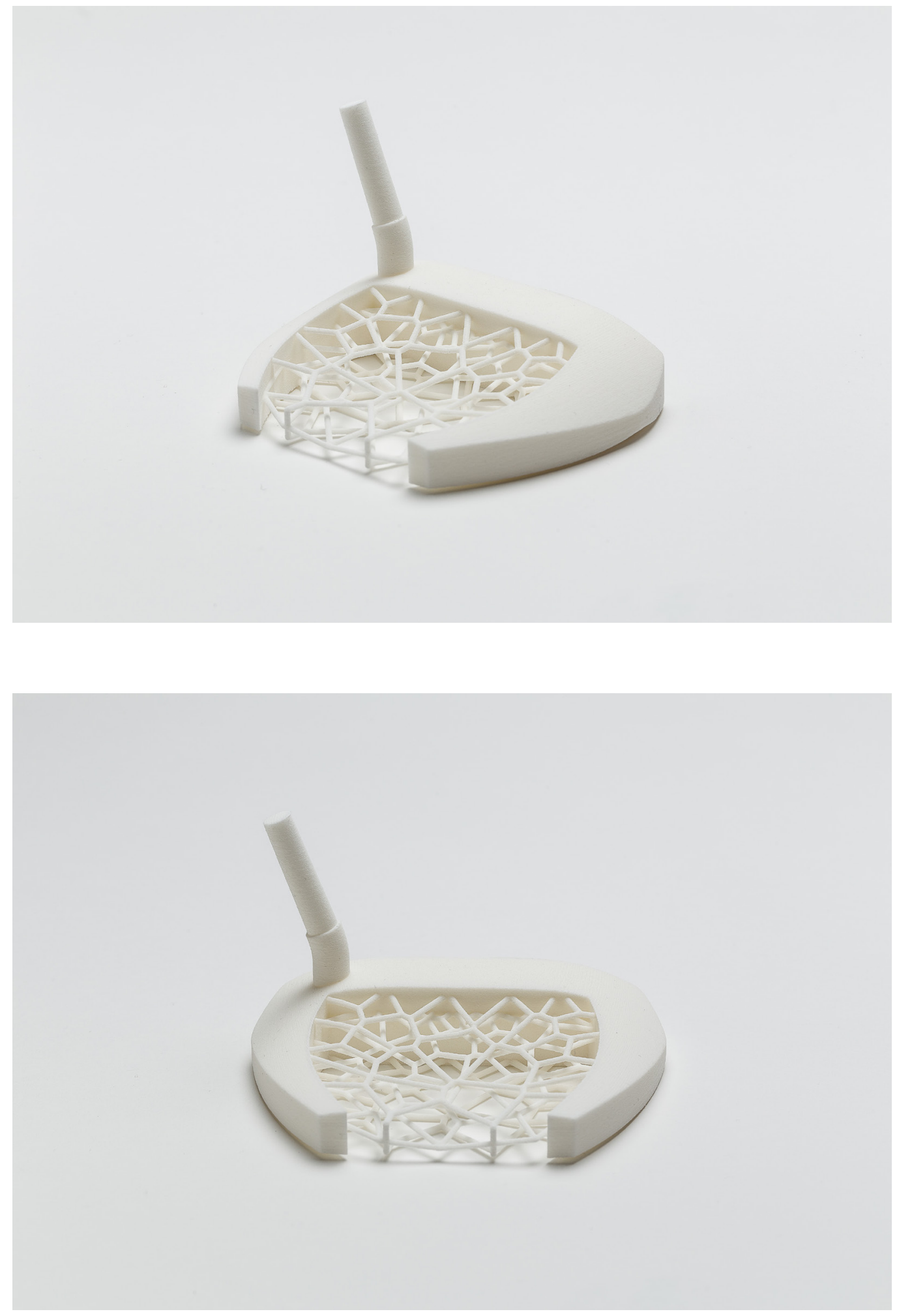
Fig. 60| P4 object and detail shots

$-169-$ 
Fig. 61| P5 function + aesthetics

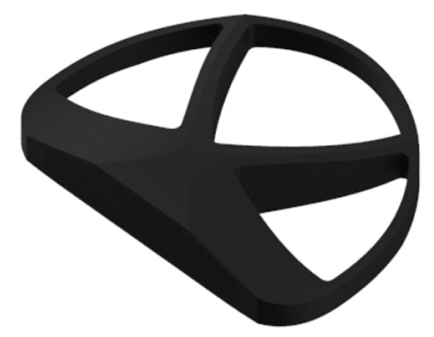

$+$

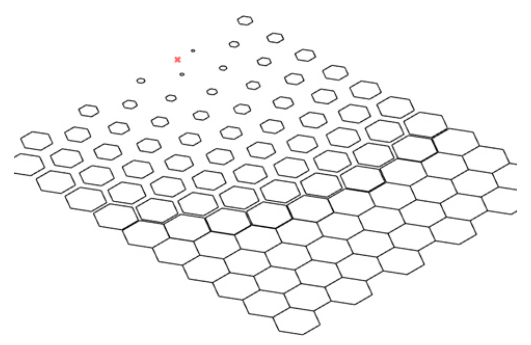

$=$

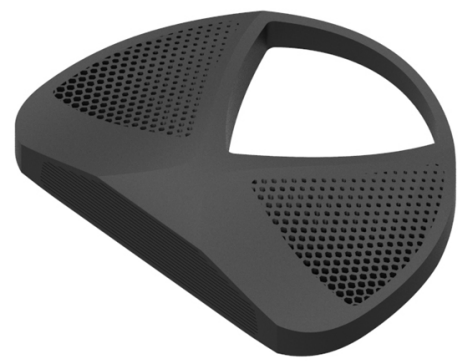




\section{PRELIMINARY FIVE}

Preliminary design five explores the functional potential of hexagon-cell panelling tools. Drawing on the findings from series two (particularly attractor surfaces 1 \& 3 ) and attractor points 3 , this experiments aims to utilize honeycomb structures to functionally distribute weight throughout a clubhead.

In a similar manner to preliminary design two this experiment addresses paneling tools for aesthetic, however the strength to weight ratio of honeycomb structures make it a superior functional choice over diamonds.

The experiment employs two fundamental parameters: the scaling of honeycomb geometry as a reflection of weight distribution and the seamless integration and blending of geometry and honeycomb aesthetic into the clubhead. The scaling of honeycomb geometry is defined by 3 attractor points.

It was found that the combination of parameters worked very well together in terms of function and aesthetic. The weight was effectively distributed relative to the data while the honeycomb structure provided added visual complexity without overpowering the club's true form. The visual language generated by computational aesthetic effectively suggests the club's true function to the user. 
Fig. 62| P5-3D printed model
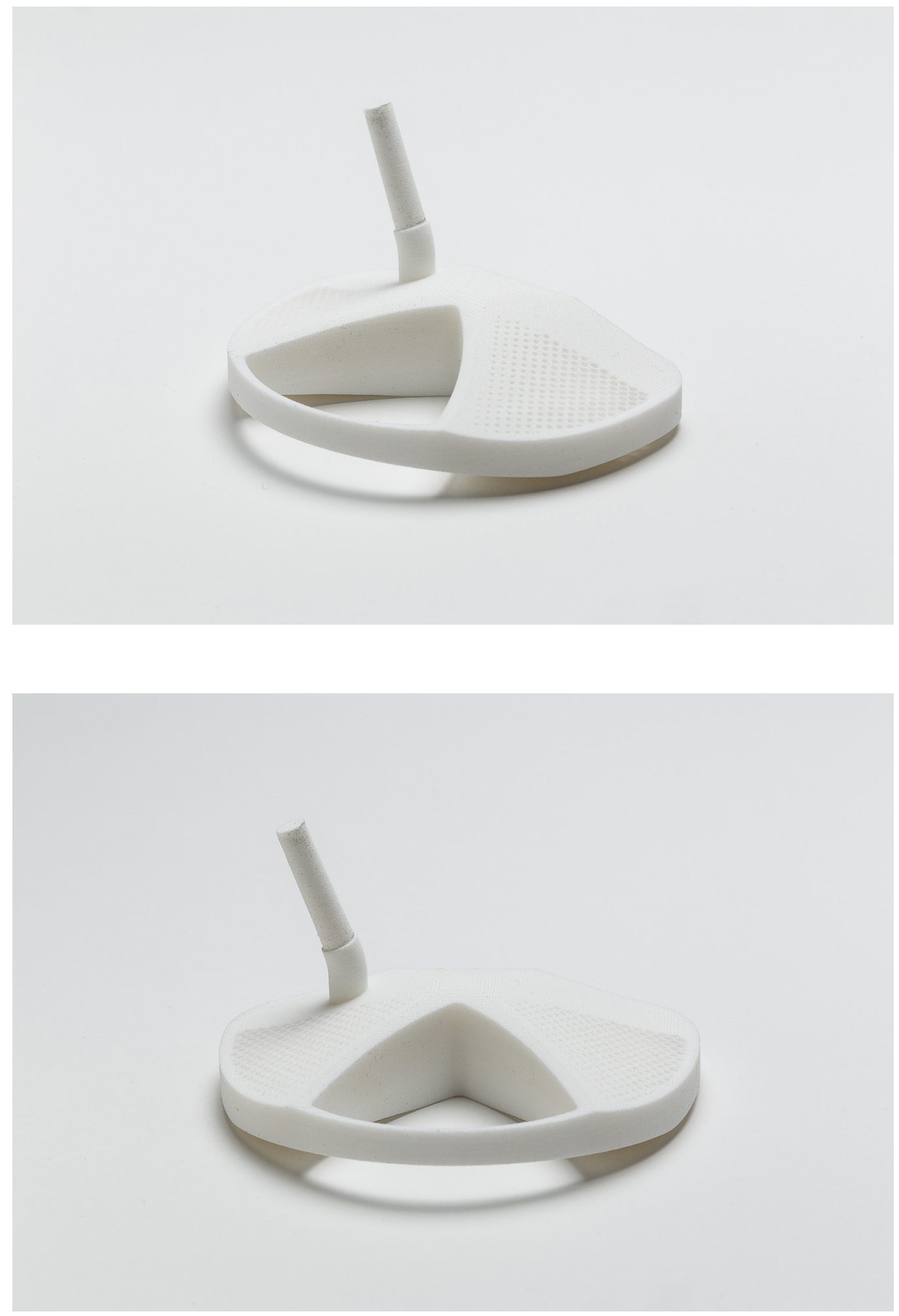
Fig. 63| P5 object and detail shots

$-173-$ 
Fig. 64| Matrix evaluation graph

\begin{tabular}{|l|l|l|l|l|l|}
\hline & WD & FC & VC & VL & IN \\
\hline P1 & 4 & 3 & 3 & 4 & 3 \\
\hline P2 & 4 & 2 & 3 & 5 & 2 \\
\hline P3 & 1 & 2 & 5 & 3 & 5 \\
\hline P4 & 2 & 2 & 5 & 3 & 5 \\
\hline P5 & 5 & 4 & 4 & 5 & 3 \\
\hline
\end{tabular}




\section{CONCLUSIONS}

Chapter seven has discussed how the application of divergent computational design aesthetics, driven by parametric modelling can enhance or degrade functional and mental performance aspects in a golf clubhead. Each design concept takes an experimental approach to using emerging digital tools. In most cases, the application of these aesthetics addressed one performance aspect particularly well but not always the other.

A matrix evaluation graph (Milton \& Rodgers, 2013) has been formulated to evaluate each preliminary design proposals against objective based criteria (fig 64). Each concept is giving a mark out of five in relation to its effectiveness to meet each of the five criteria, these are:

- Weight distribution (WD) in relation to data

- Functional criteria (other than WD such as MOI \& COG)

- Visual complexity

- Visual language (ability to communicate function)

- Innovation (value is neutral)

Based on the results of this graph, preliminary design five has been selected to develop further. 


$$
2=2
$$


$\leq b$ 


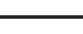

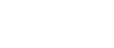


CHAPTER EIGHT

DEVELOPED DESIGN 


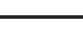

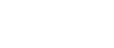




\section{DEVELOPED DESIGN}

Chapter eight aims to draw on the collective knowledge of previous experiments and further refine clubhead performance aspects, particularly function driven features. This chapter aims to produce a fully functional and tangible object that can manufactured and applied effectively in context. This means that issues such as material choice and its relation to data affected geometry must be considered. The use of data for functional purposes is also addressed. In previous experiments data and relative parameters have been assigned to functional performance aspects based on rough calculations and relatively small fields of data. In this chapter data will be determined over a larger field, producing a more accurate representation of the input for functional application. 
Fig. 66 | Comparable weight distribution
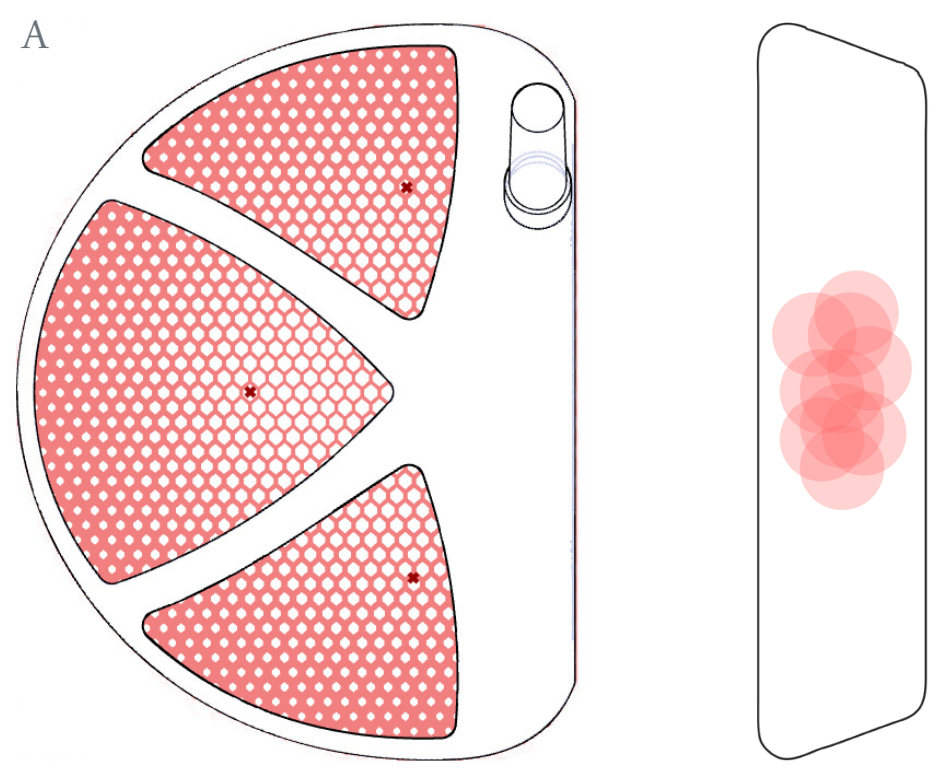

B
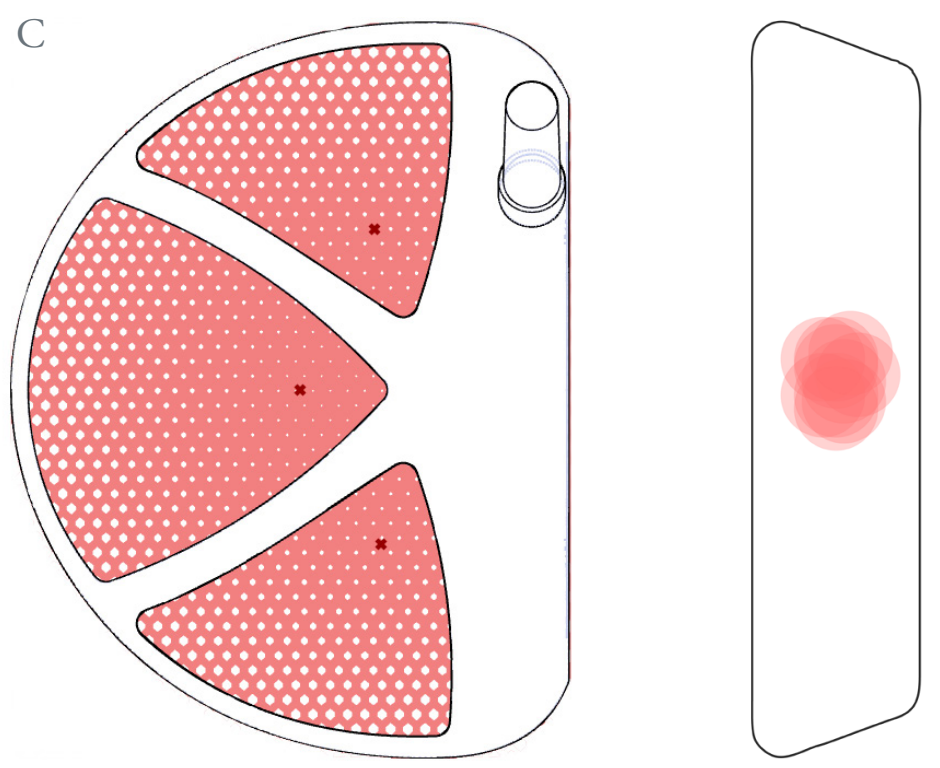

$\mathrm{D}$ 


\section{DATA REFINEMENT}

As mentioned in chapter one, the distribution of weight is an essential aspect to consider when designing a clubhead. A precise, calculated distribution suited to the specific player's stroke style can be vital in the failure or success of a design.

This section explains how parameters for distributing weight amongst a clubhead work in relation to said data. Comparisons are made between two different stroke styles, to emphasize the importance of correct weight distribution.

Fig 66 exhibits an example where the weight is predominantly distributed around the perimeter of the clubhead (a). This style of putter is typically assigned to a golfer who possesses an inconsistent putting stroke (amateur golfer). In other words, the ball tends to be struck all over the face (b). As mentioned earlier, this means the club is prone to twisting, resulting in a off line putt. A perimeter weighted club counteracts this by creating by evenly distributing weight around the club, offering an even balanced club that is less prone to twisting. However a perimeter weighted club offers less distance and direction control.

Fig 66 also exhibits a comparable example where the weight is predominantly centred (c). This style of putter is typically signed to a golfer with a more consistent (but not perfect) putting stroke. In other words, the ball is struck more consistently across the face $(\mathrm{d})$. 
Fig. 67 | Iterative data customisation

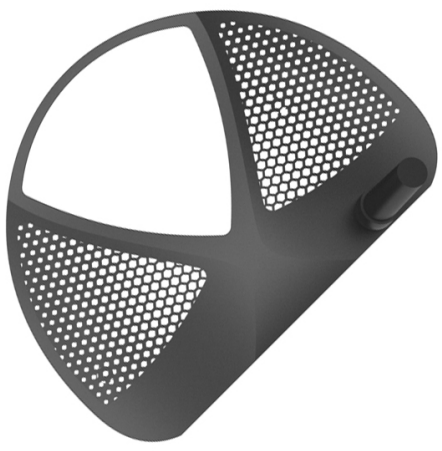

1

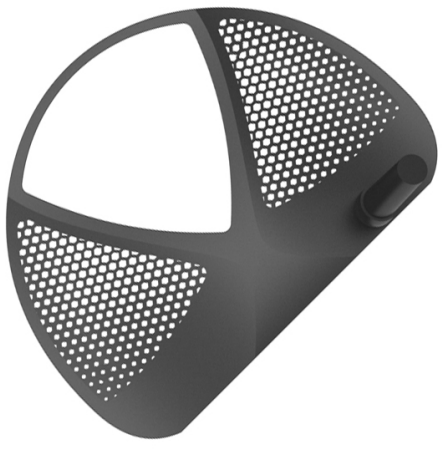

20

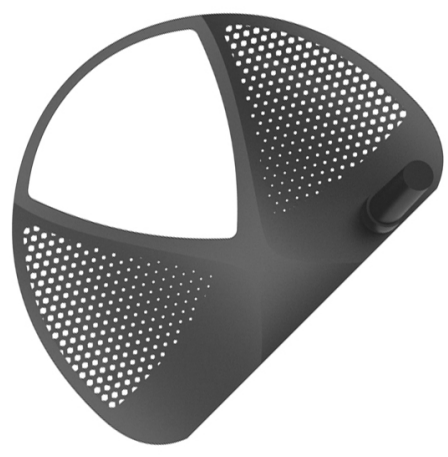

58

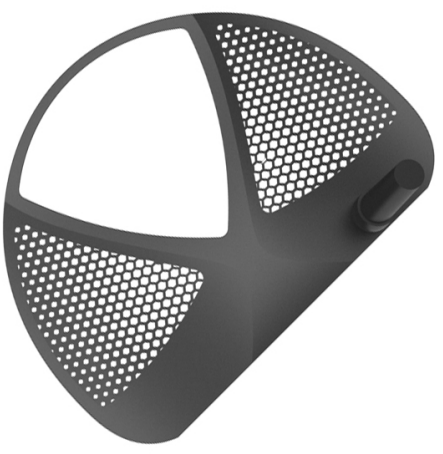

8

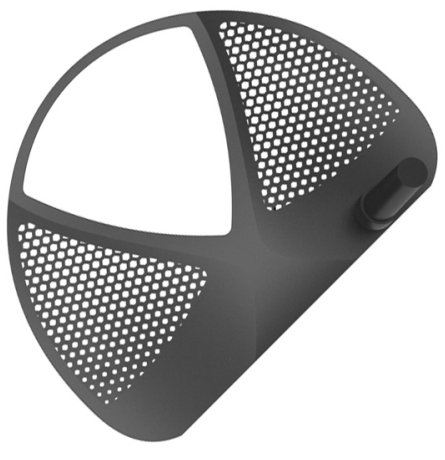

25

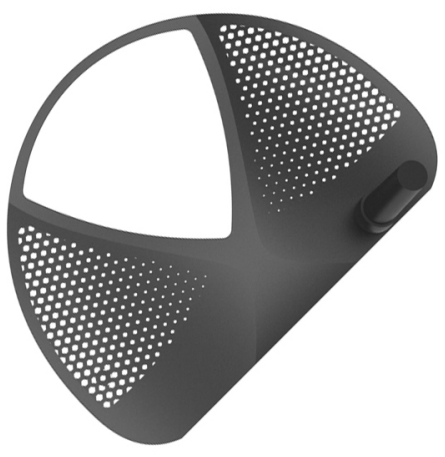

65

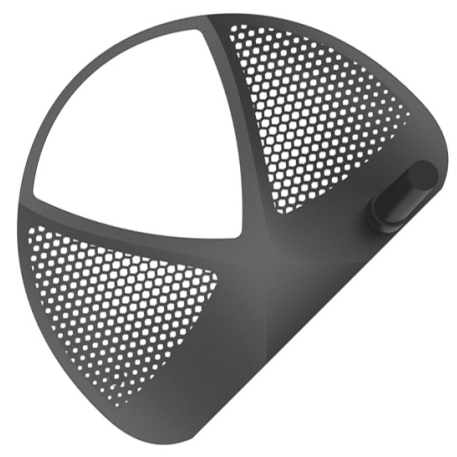

15

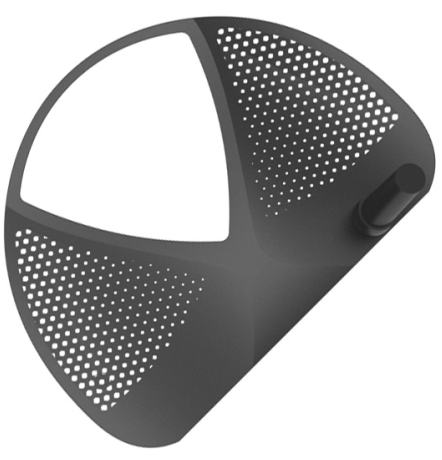

50

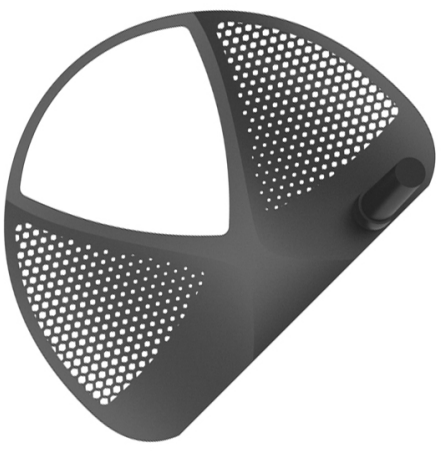

72 
A centre weighted club is more prone to twisting on an off centre hit, however if struck more consistently from the middle, offers more distance and direction control.

In relation to a parametric model, the position of attractor points (dark red dots (fig 66, a \& c)) determines where the weight is distributed from. The location of that point and size of the hexagonal openings are defined by the consistency of ball striking on the face which is determined by the player's stroke type, impact angle and tempo as discussed in the programme analysis.

Fig 67 provides an insight into how a larger field of data (more strokes applied to an average) produces a more refined geometry, with more accurate customised individual stroke data.

Iteration 1 presents the affect of the first putting stroke data on the weight distribution. After it each putt is made, the average of data changes. Note: the gap between iterations 25 and 50 (larger than usual) for comparison.

Iteration 72 represents the last parametric iteration of that club geometry. At this point a consistency has been reached the point where the next normal stroke no longer affects the average of data recorded. At this point, determined by the parameters set, this club is ready for fabrication. 
Fig. 68 | Exploded view, developed design

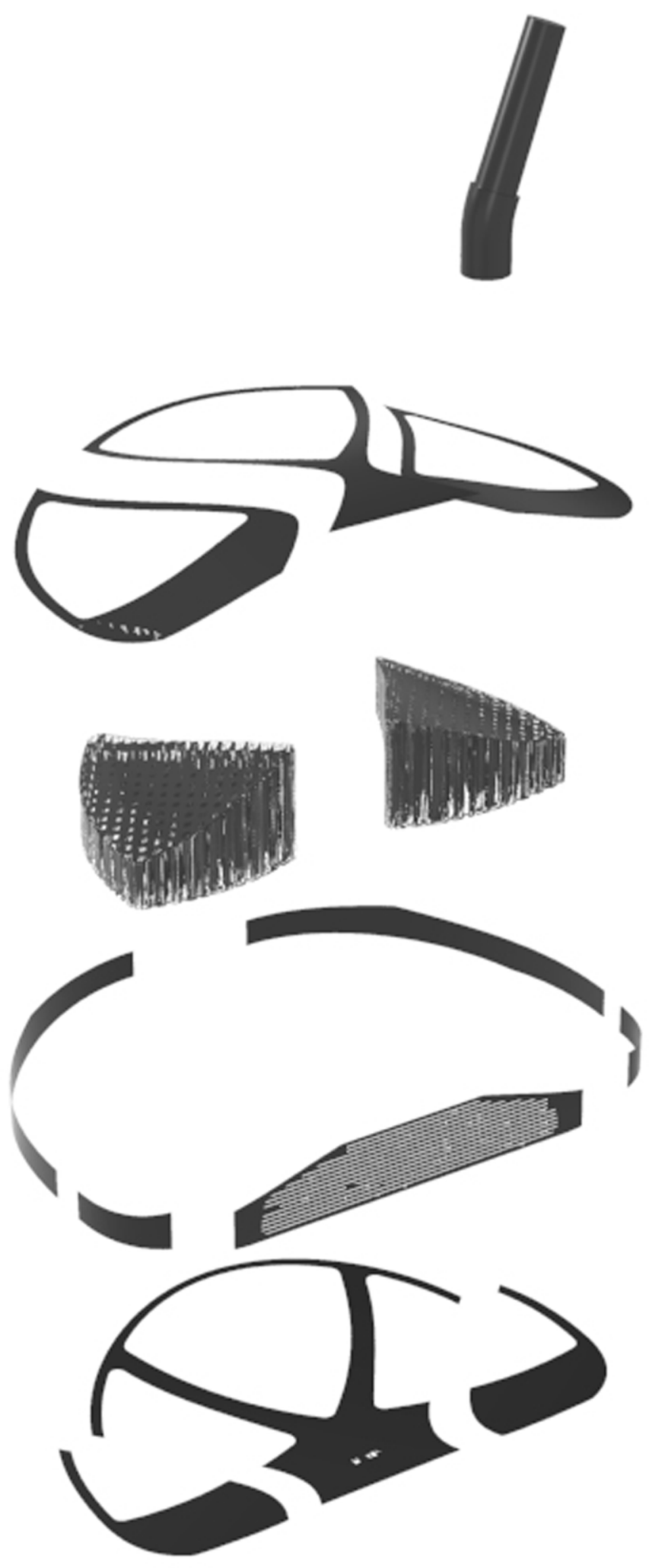




\section{MATERIALS}

The developed design is now a tangible object which has been intentionally constructed to be manufactured as a 1:1 scale. This model, also fabricated at Shapeways, was printed in a matte black stainless steel. As described by Shapeways (Shapeways, 2015), their material is a "robust metal, with a rough pitted surface." Made of 420 stainless steel infused with bronze, and has a final composition of approximately $60 \%$ steel and $40 \%$ bronze. The material is also watertight, rendering it as functional for all weather conditions in context. 
Fig. 69| Cavity shot, developed design

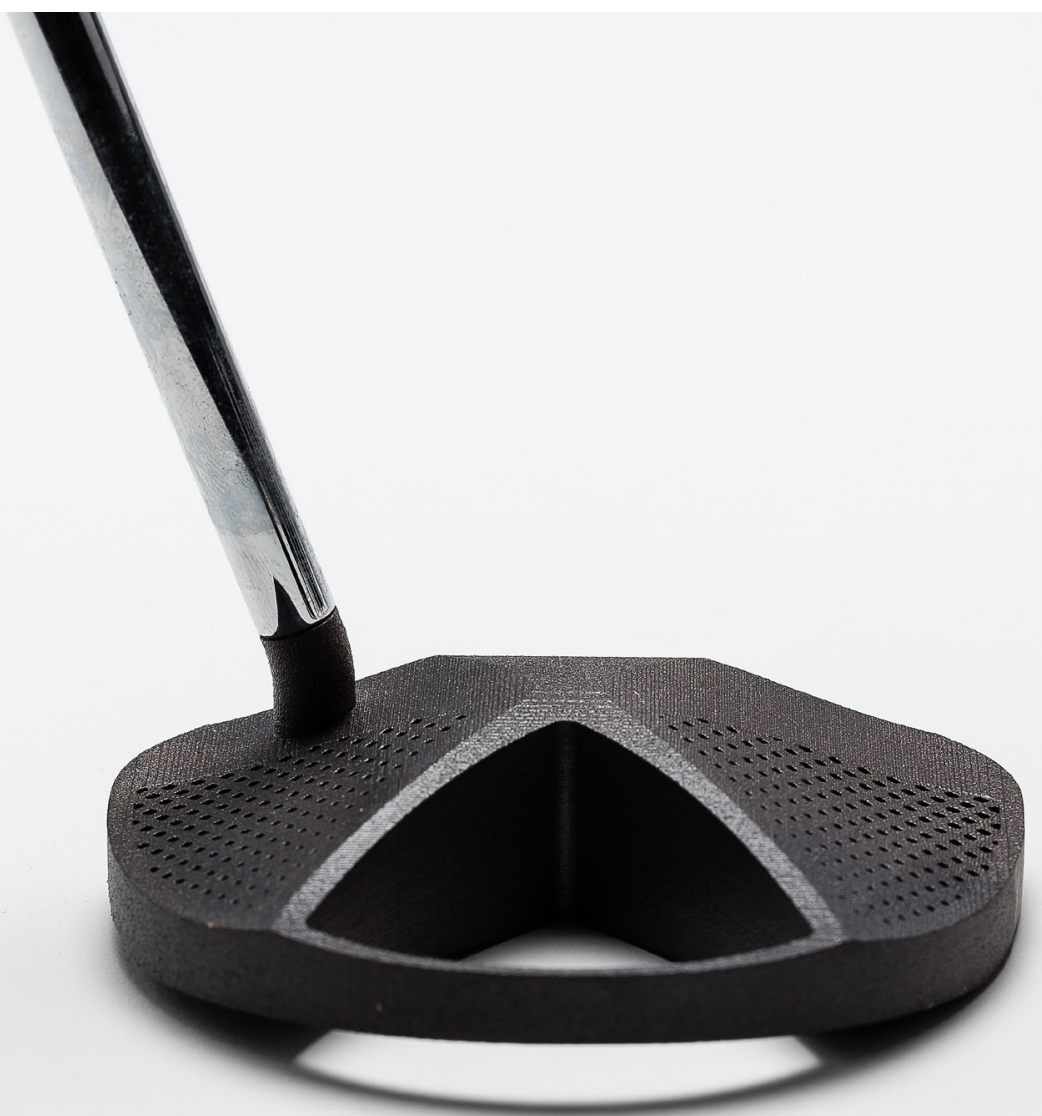


Fig. 70 | Weight distribution detail, developed design

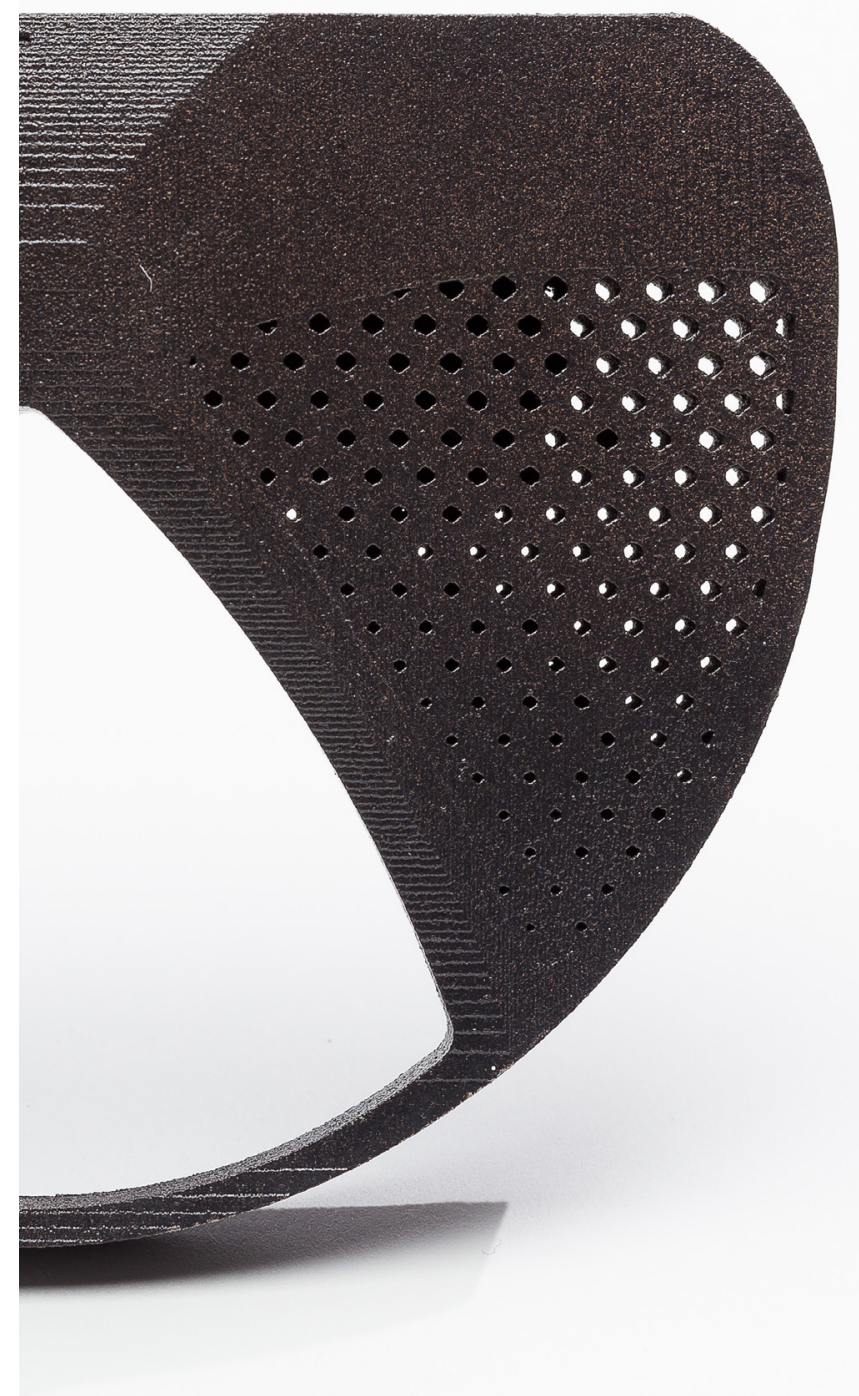




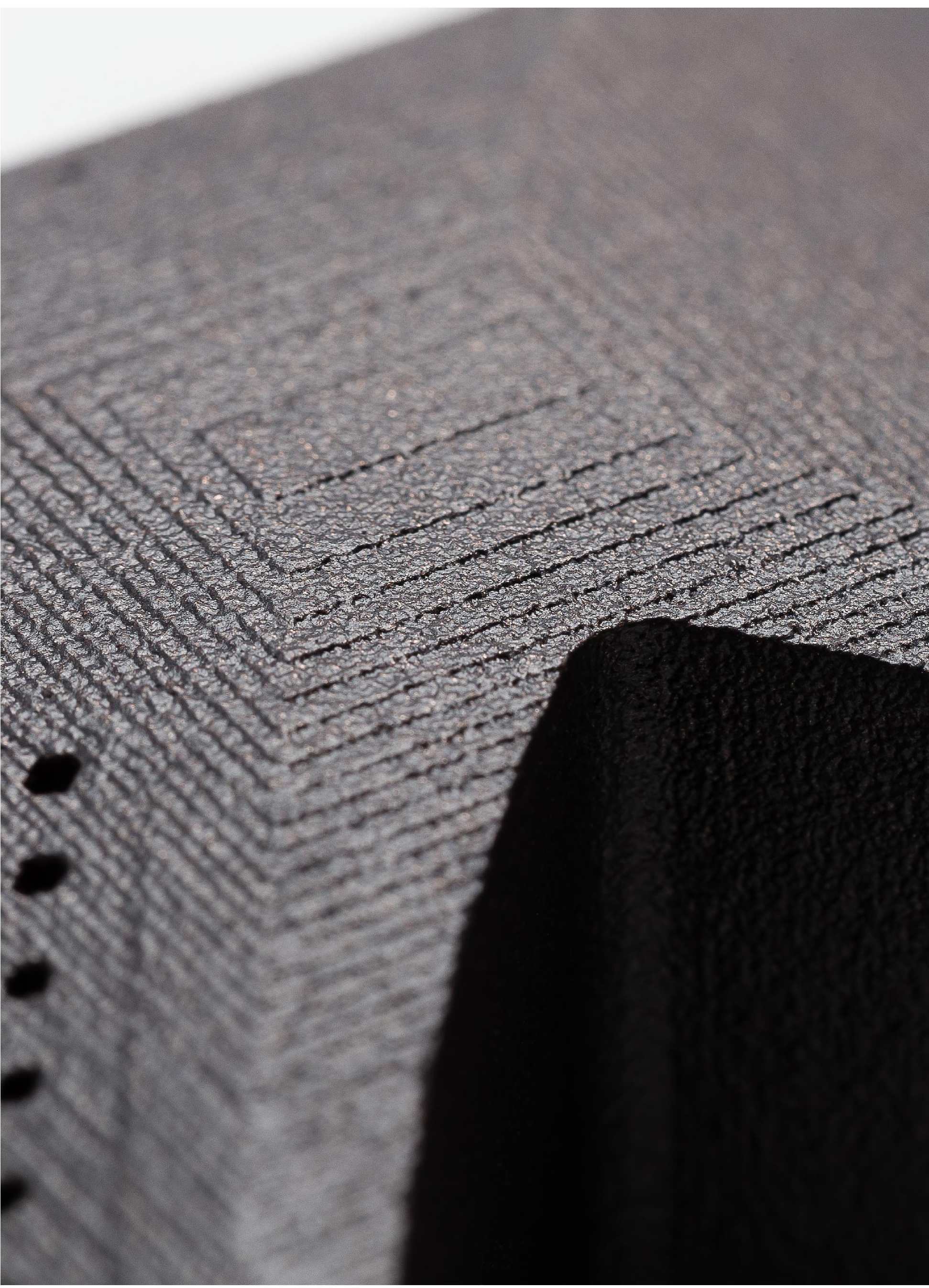


Fig. 72 | Shadow detail, developed design

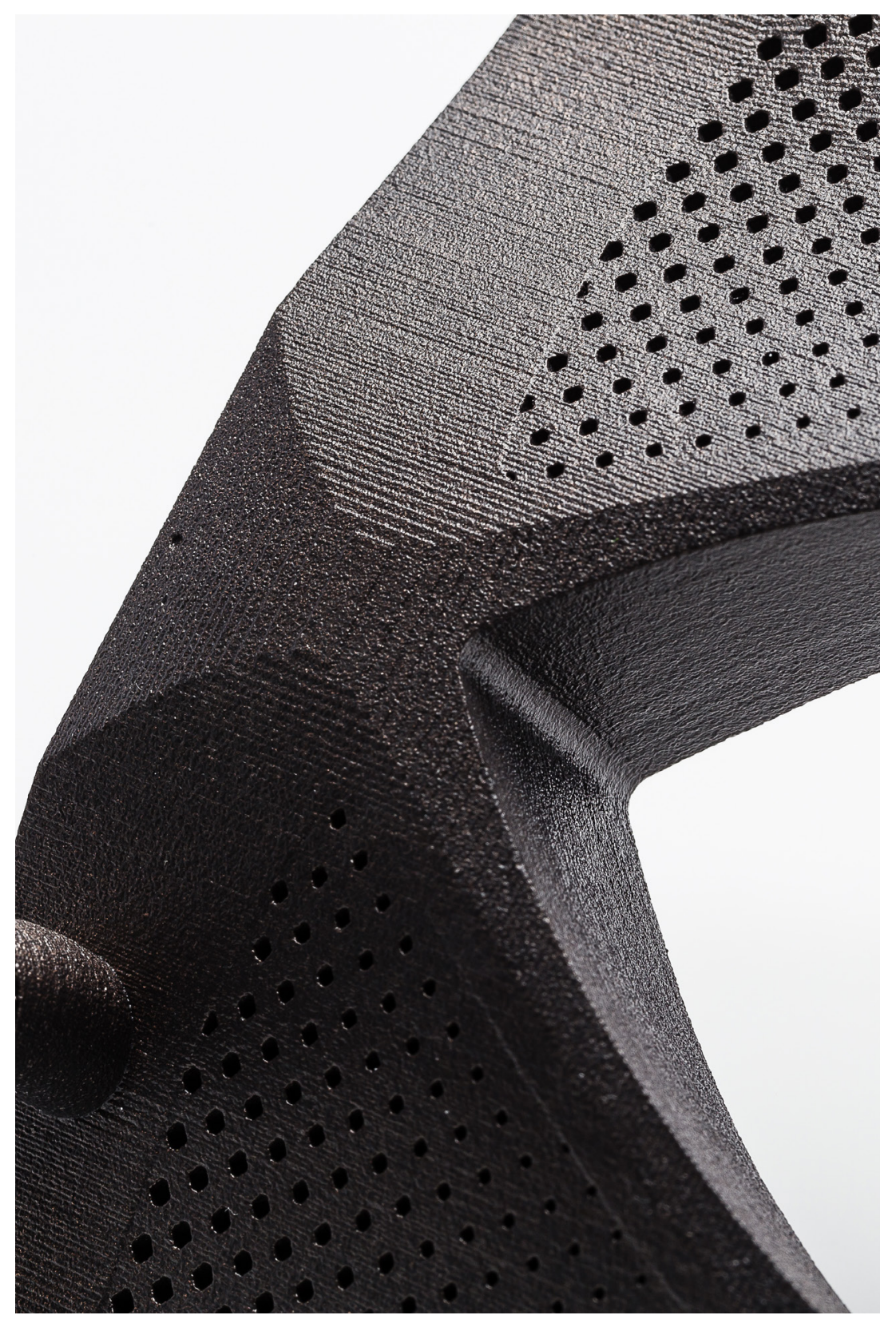


Fig. 73 | Face details, developed design
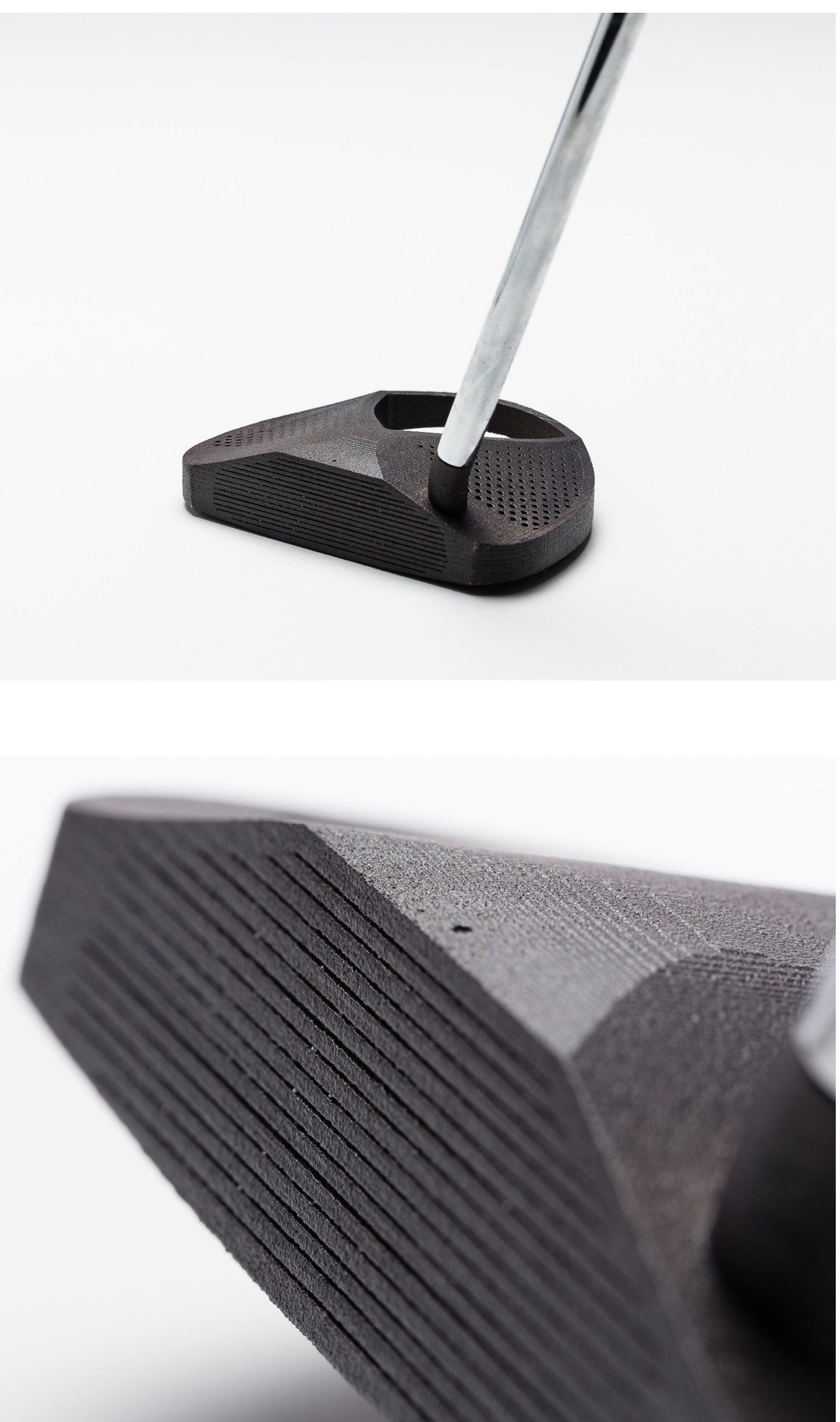


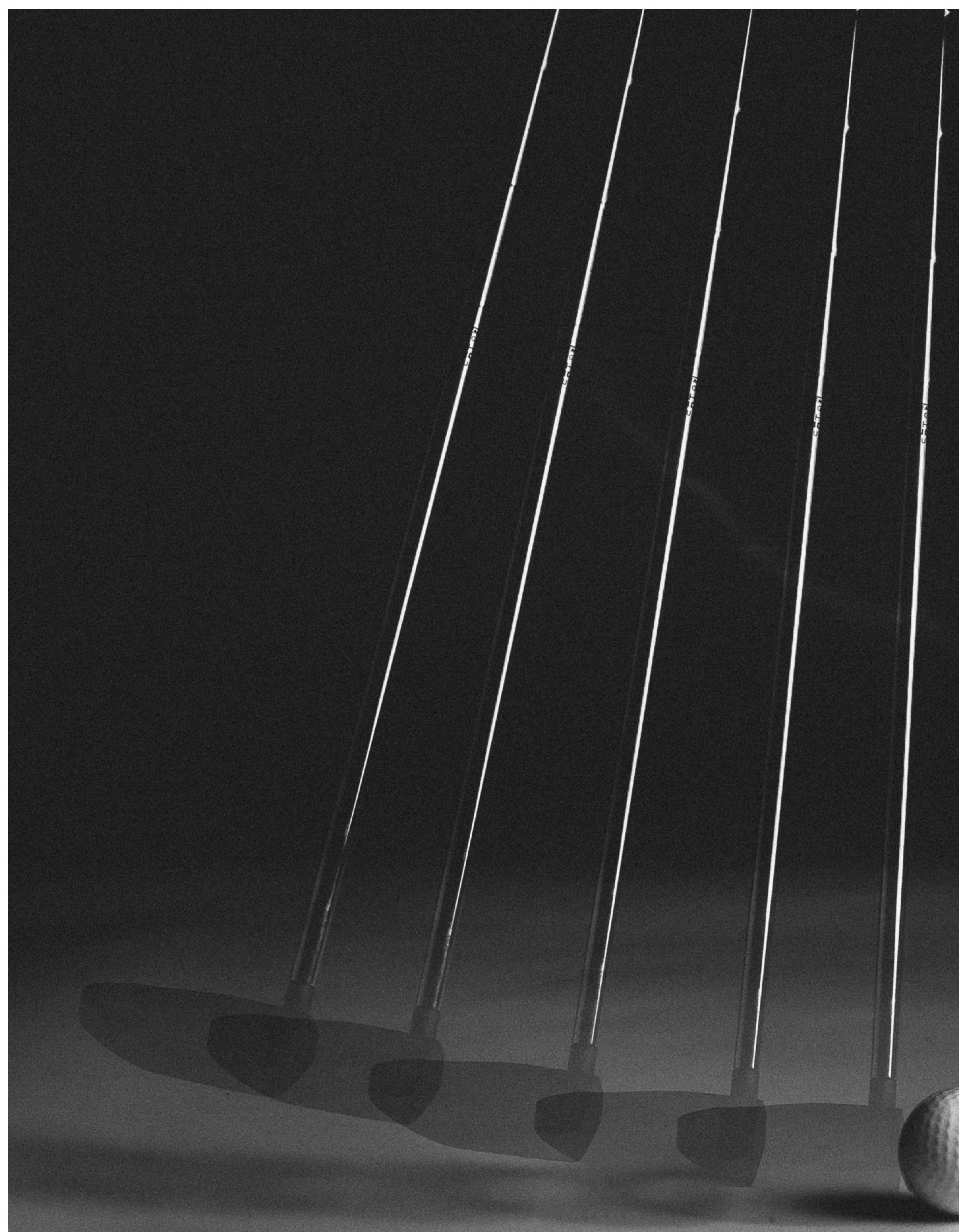


Fig. 75 | Players stroke view, developed design

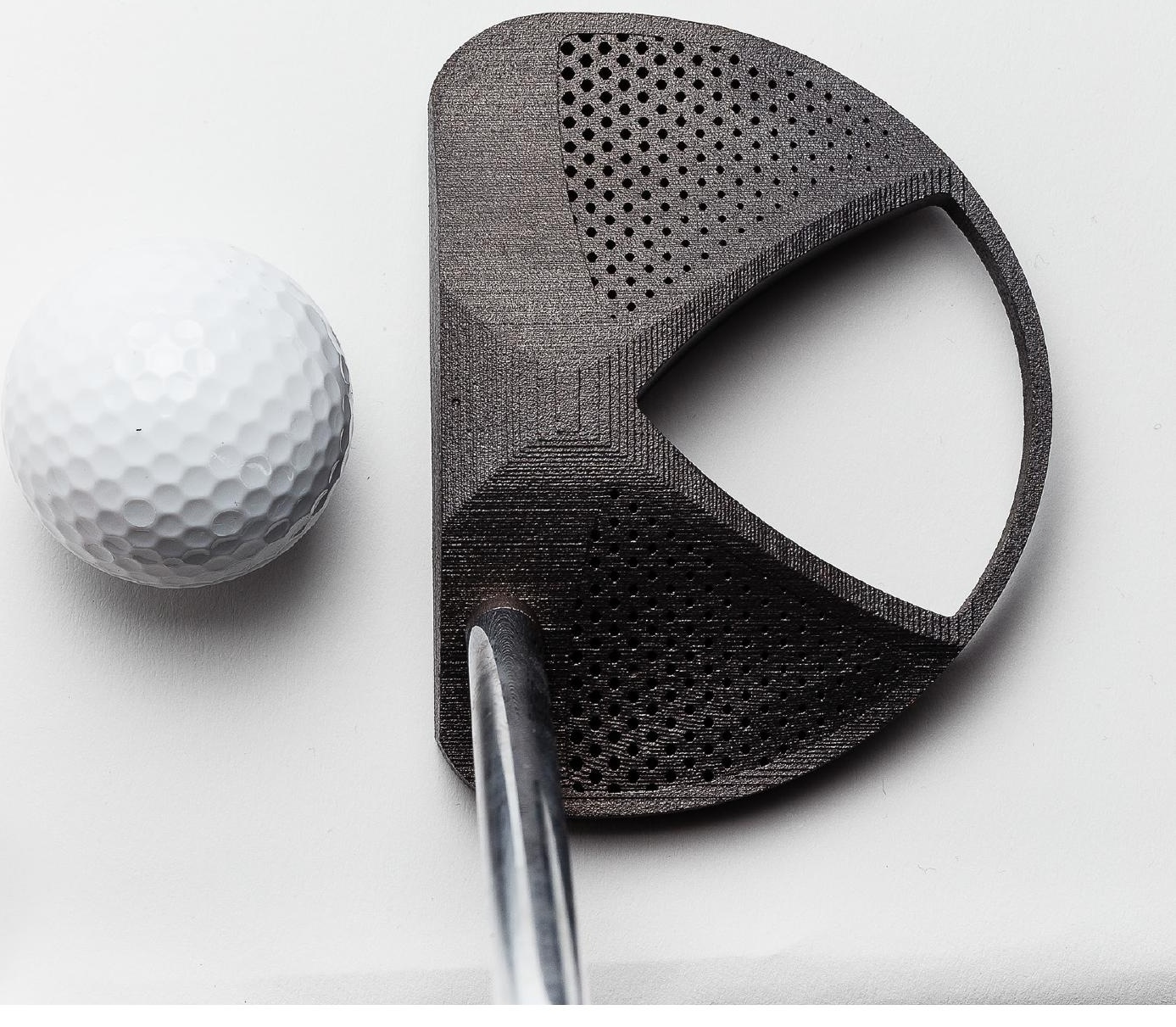


Fig. 76 | Context shot, developed design

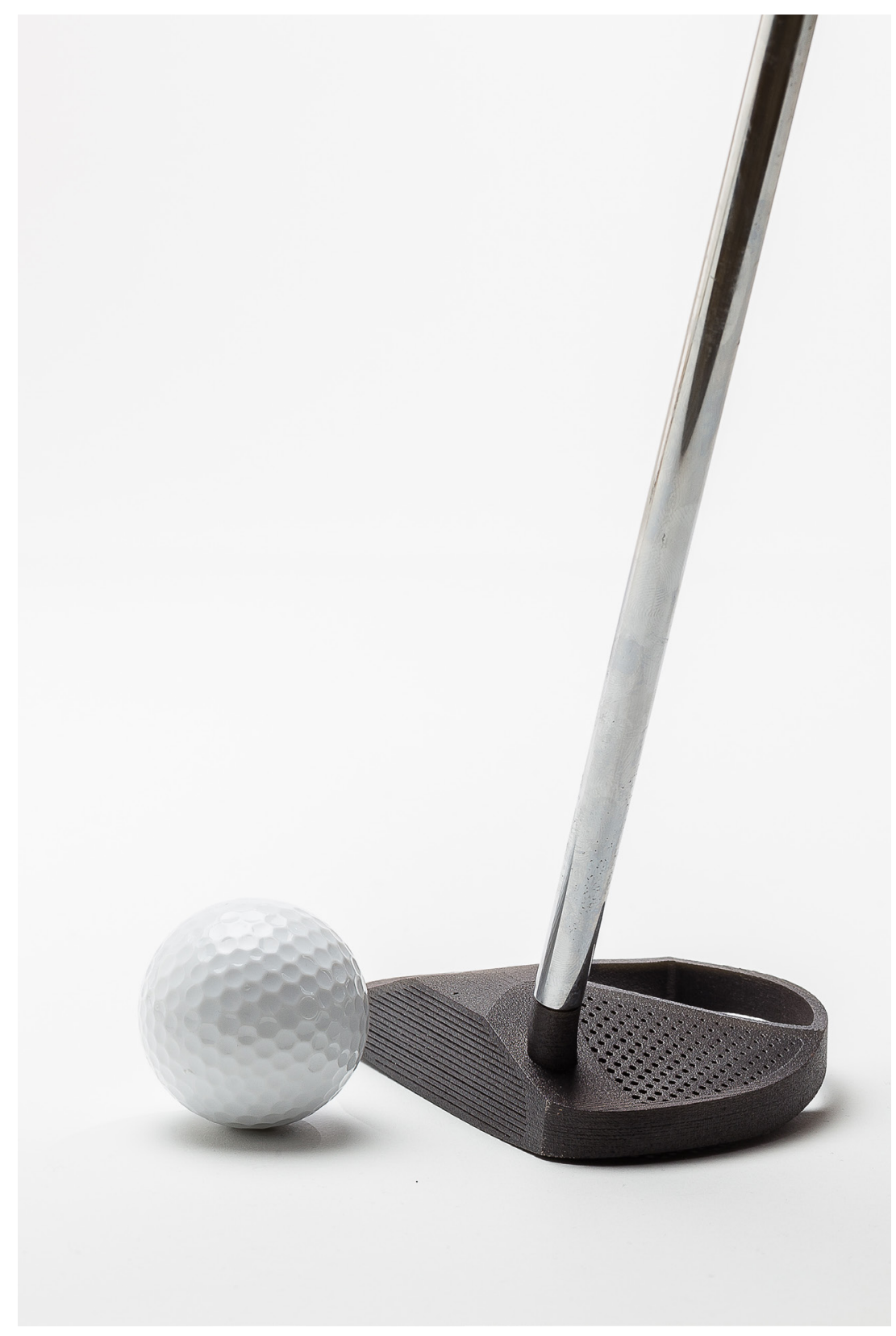


Fig. 77 | Context shot, developed design

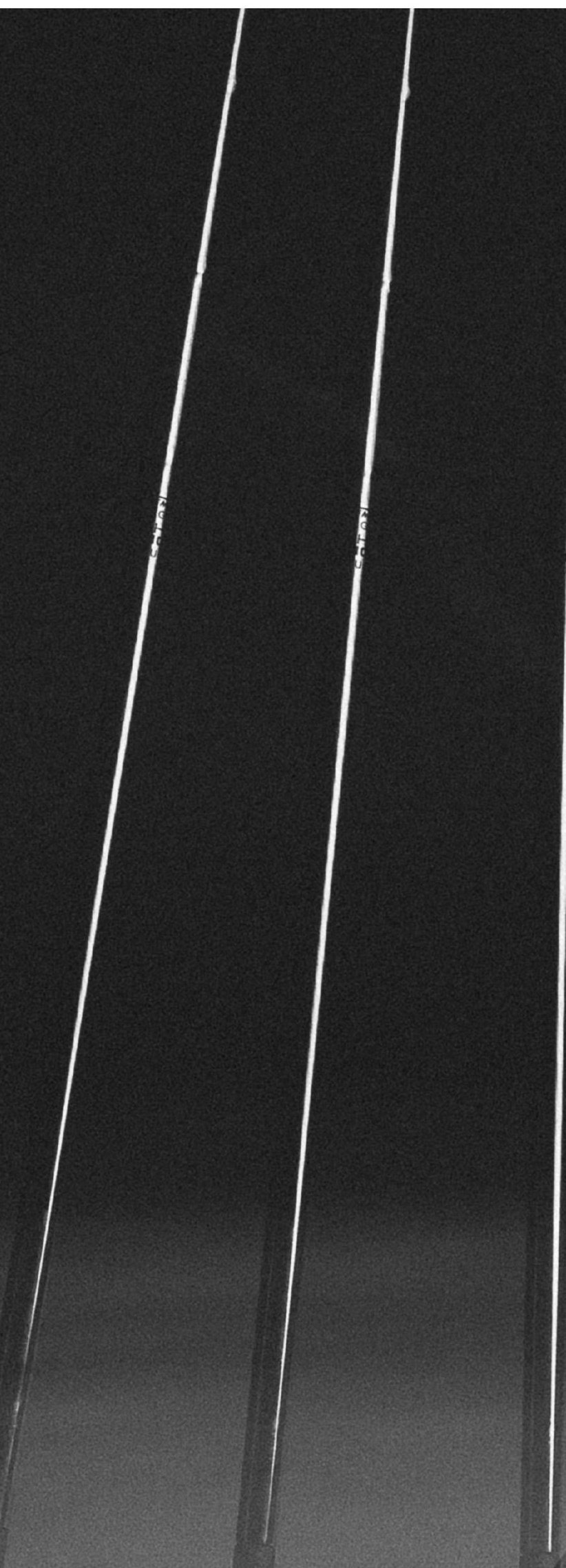




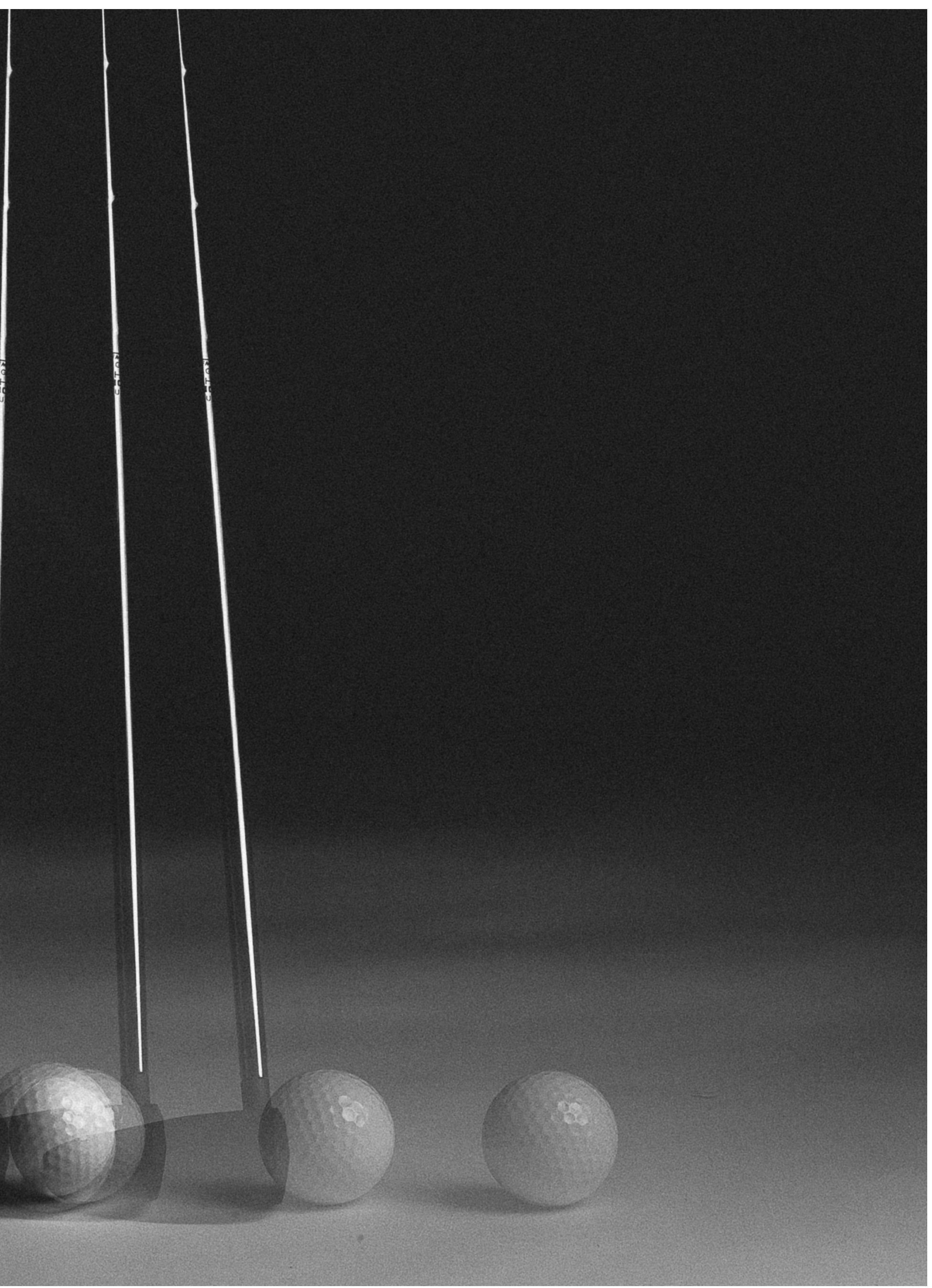




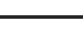

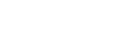




\title{
CHAPTER NINE
}

\author{
DISCUSSION
}


Can the integration and synchronisation of contemporary digital technologies reshape the design process of golf clubs?

Within the area of sports equipment design, there will never be the perfect design solution. The nature of sport won't allow it. Science won't stop finding, engineers won't stop perfecting and athletes won't stop striving to be the best. Whether it's a swimmer's wetsuit, the strings on a tennis racket or the head of a golf club, the continual development of materials, technologies and processes mean the role of the designer and how they utilize these resources is more important than ever.

This research has strived to be the next revolutionary step in the evolutionary design process that is golf club design. It has explored the impact that emerging technologies can have on the traditional design process. The prime objectives of this experimental exploration were to propose design solutions that; integrated individual performance data as form defining inputs; explored the potential of new aesthetics to enhance functional criteria; and preserved critical features of traditional club design.
The conclusions drawn from this investigation suggest that the integration and synchronisation of contemporary digital technologies is a step in the right direction towards reshaping golf club design. The power and variation these technologies offer seems almost limitless, and this exploration, without being overbearing, becomes another important step before the next, in the evolutionary design process.

It must be said that this boundless approach to design makes the designers role more critical than ever. The sheer variation of considered decisions the digital designer must make can determine the object's validity. The experimental, iterative research through design approach proved an effective method, alongside the designers vision to exploit these technologies in making informed design decisions.

Ideas and methods on how to incorporate individual data into an object, how to drive function through aesthetic and how to cater for adaptable inputs were derived from research conducted in the project and literature reviews. Providing a new lens in which 
to establish a meaningful and purposeful foundation to drive design experiments.

Integrating individual, differentiated swing data as a meaningful design input during the design process made for an effective method for enhancing the customisation of a golf clubhead. It not only improves mental and physical performance aspects of a putter but it strengthens the relationship between human and object.

One of the most interesting developments of this investigation was the way in which aesthetics were used to enhance functional performance criteria. All sports equipment is designed with the underlying theme of improving performance in some way, shape or form. In most cases a team of scientists and engineers will inform a designer of functional materials, technologies and processes they could use to explore new aesthetics. This research takes a different approach. Instead, it utilizes digital aesthetics to explore new function (new means of enhancing function). The decision to explore computational design aesthetics through a range of digital tools proved to be an effective means of reshaping what could be seen as functional criteria.

The predominant constraints and limitations of this research were the availability of particular resources such as; the quality and purpose of data collection tools (perhaps using a device specifically designed to record modelling inputs, rather than the designer analysing and interpreting the data); and the access and price of metal additive manufacturing methods.

With the appropriate resources, the methods and processes used in this research could be easily extended toward other golf clubs, such as wedges, irons or drivers. In fact, this approach could be applied to any performance driven sports equipment, whether it be a bicycle or a cricket bat. The nature of these tools puts the only limitations in the designers hands.

One thing's for sure though, we are closing the gap between human and object. 


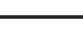

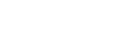




\section{LIST OF FIGURES}

Additional images not listed are authors own. All photography in chapters $7 \& 8$ was commissioned by author to Saint Andrew Matautia. 
Figure 1. Burrows, M. (1992). Lotus type 108 bicycle [online image]. Retrieved July 1, 2015 from http://www.core77.com/posts/22205/design-museum-londonsupcoming-exhibition-examines-the-intersection-of-sports-and-design-22205

Figure 2. Clisthenes in the Olympic games [illustration]. Retrieved February 2015 from http://images.fineartamerica.com/images-medium-large/ancientolympic-games-granger.jpg

Figure 3. Golf in the late 1800's [illustration]. Retrieved July 2, 2015 from https://alexanderseamon.wordpress.com/2013/04/29/early-golf-and-todays-golflifestyle/

Figure 7. Ping. (1972). Ping colour code chart [online image]. Retrieved January 2015 from https://s-media-cache-ak0.pinimg.com/originals/cf/61/fa/ cf61 fab 108c807b23c4394d21924dc68.jpg

Figure 8. Swingbyte. (2014). Swingbyte attached to club shaft [online image]. Retrieved October 2014 from http://ecx.images-amazon.com/images/ I/71TGixSTYwL._SL1500_.jpg

Figure 10. Widrig, D. (2012). Grid detail shot [online image]. Retrieved August 2014 from http://www.danielwidrig.com/index.php?page=Work\&id=Grid

Figure 11. Zaanen, J. (2014). 3D printed putter prototype [online image]. Retrieved February 2015 from http://www.golfwrx.com/forums/topic/1015729$3 \mathrm{~d}$-printed-putter-prototypel

Figure 12. Ping. (2015). 3D printed putter [online image]. Retrieved June 2015 from http://3dprint.com/46036/golf-equipment-manufacturer-ping-introducesgolfs-first-3d-printed-putter 
Figure 13. Shiro Studio. (2013). Trabeculae detail [online image]. Retrieved July 2014 from http://www.shiro-studio.com/trabeculae.php

Figure 14. Nervous System. (2014). Hyphae 3D 1 [online image]. Retrieved May 2015 from http://n-e-r-v-o-u-s.com/projects/albums/growing-objects/content/ hyphae-3d-1/

Figure 15. Matter Design. (2013). Knotta Chandelier [online image]. Retrieved January 2015 from http://www.matterdesignstudio.com/knotta/

Figure 16. Morgante, A. (2013). 25.0 collection [online image]. Retrieved July 2014 from http://www.dezeen.com/2014/07/22/andrea-morgante-3d-printedperoni-piccola-bottles/

Figure 17. Widrig, D. (2012). Brazil chair [online image]. Retrieved July 2014 from http://www.danielwidrig.com/index.php?page=work\&id=brazil_no2

Figure 18. Arup. (2014). 3D printed steel nodes [online image]. Retrieved Jan 2015 from http://www.arup.com/News/2014_06_June/05_June_Construction_ steelwork_makes_3D_printing_premiere.aspx

Figure 19. Widrig, D. (2011). Kinesis, 3D printed wearable sculpture [online image]. Retrieved July 2014 from http://www.danielwidrig.com/index. php?page $=$ Work\&id $=$ Kinesis

Figure 31. Klein Project. (2015). Voronoi patterning showing points of attraction [online image]. Retrieved March 2015 from http://blog.kleinproject.org/?p=742 


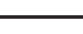

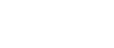


REFERENCES

$-209-$ 
Bohn, M. K. (2007). Money Golf: 600 Years of Bettin' on Birdies. Potomac Books, Inc.

DeSimone, J. (2015). What if 3D printing was $100 \mathrm{x}$ faster? TED talk. Retrieved from http://www.ted.com/talks/joe_desimone_what_if_3d_ printing_was_25x_faster?language $=$ en

Dormer, P. (1997). The Culture of Craft. Manchester University Press.

Drimmer, A. (2014, April 24). The secret history of Lotus's first twowheeler. Retrieved May 12, 2015, from http://www.roadandtrack.com/ features/web-originals/lotus-sport-108-secret-history-bicycle

Ellis, J. B. (1997). The clubmaker's art: antique golf clubs and their history. Oak Harbor, Wash: Zephyr Productions.

Epstein, D. (2014). Are athletes really getting faster, better, stronger? Retrieved from http://www.ted.com/talks/david_epstein_are_athletes_ really_getting_faster_better_stronger?language $=$ en

Gaijaard, S. (2014). 3D-printed structural components will lead to "new building shapes." Retrieved January 30, 2015, from http://www.dezeen. com/2014/12/17/movie-3d-printed-building-components-new-buildingshapes-salome-galjaard-arup-video-interview/

Godin, D., \& Zahedi, M. (2014). Aspects of Research through Design: A Literature Review. In Proceedings of DRS 2014: Design's Big Debates. Sweeden. Retrieved from http://www.drs2014.org/media/648109/0205file1.pdf 
Hoffman, C., \& Joan-Arinyo, R. (2002). Parametric Modelling. Handbook of CAGD, 519-541.

Jenkins, M. (2009, May 5). The Advantages of Titanium Golf Clubs [Golf Link]. Retrieved from http://www.golflink.com/facts_597_advantagestitanium-golf-clubs.html

Kilkelly, M. (2015, April 13). Are Computers Bad for Architecture? Retrieved May 4, 2015, from http://www.archdaily.com/618422/arecomputers-bad-for-architecture/

Maltby, R. (2006). How To Properly Fit A Golfer For Putters. Retrieved from http://ralphmaltby.com/how-to-properly-fit-a-golfer-for-putters/

Matter Design. (2013). Knotta Chandelier, Matter Design. Retrieved January 27, 2015, from http://www.matterdesignstudio.com/knotta/

Milton, A., \& Rodgers, P. (2013). Research methods for product design. London: Laurence King Pub.

Morgante, A. (2014). Andrea Morgante abstracts Peroni beer bottle into 3D-printed shapes. Retrieved January 26, 2015, from http://www.dezeen. com/2014/07/22/andrea-morgante-3d-printed-peroni-piccola-bottles/

Noble, I., \& Bestley, R. (2005). Visual research an introduction to research methodologies in graphic design. Lausanne: AVA. Retrieved from http:// site.ebrary.com/id/10443168

Nurse, M. (2014). A Look Inside Nike's Sport Research Lab. Retrieved from http://news.nike.com/news/a-look-inside-nike-s-sport-research-lab 
PING. (2009). Custom Fitting. Retrieved from http://pinggolfblob.ping.com/pinggolf/uploadedfiles/videos/history/2009/ customfitting_655x368.mp4

Ray, P. (2011). Design and personalization of golf club heads using electron beam melting, functionally graded porosity, and numerical analysis (M.S.). Southern Methodist University, United States -- Texas. Retrieved from http://search.proquest.com.helicon.vuw.ac.nz/docview/915643532/ abstract? accountid $=14782$

Shapeways. (2015). Stainless Steel 3D Printing Material Information Shapeways. Retrieved July 16, 2015, from https://www.shapeways.com/ materials/steel

Shillito, A. M. (2013). Digital crafts: industrial technologies for applied artists and designer makers. London: Bloomsbury.

Solheim, J. (2015). iPing fitting. Retrieved July 13, 2015, from http:// www.ping.com/fitting/iping.aspx

Stetina, H. (2013, December 15). The Ultimate Putting Program (Part 2): Putter weight affects speed [GolfWRX]. Retrieved from http://www. golfwrx.com/151102/the-ultimate-putting-program-part-2-putterweight-affects-speed/

Sullivan, L. (1896). The tall office building artistically considered. 
Retrieved from https://archive.org/details/tallofficebuildi00sull

Turrin, M., Buelow, P. Von, \& Stouffs, R. (2011). Design explorations of performance driven geometry in architectural design using parametric modeling and genetic algorithms. Advanced Engineering Informatics, 25(656-675). Retrieved from http://www.sciencedirect.com/science/ article/pii/S1474034611000577

USGA. (2015). Rules and Decisions of Golf clubs. Retrieved May 4, 2015, from http://www.usga.org/rules/rules-and-decisions.html\#!rule-04

Weisstein, E. W. (1999). Voronoi Diagram [Text]. Retrieved July 14, 2015, from http://mathworld.wolfram.com/VoronoiDiagram.html

Widrig, D. (2010). Brazil by Daniel Widrig. Retrieved January 29, 2015, from http://www.dezeen.com/2010/05/11/brazil-by-daniel-widrig/

Widrig, D. (2013, December 5). Daniel Widrig creates wearable sculptures based on a 3D scan of the body. Retrieved from http://www.dezeen. com/2013/12/05/weird-wearable-sculptures-based-on-a-3d-scan-of-thebody-by-daniel-widrig/ 


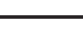

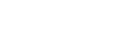


FIN

- 